\title{
ALPHABETICAL LIST OF DUTCH ZOOLOGICAL CABINETS AND MENAGERIES
}

\author{
BY \\ H. ENGEL \\ Curator of the Zoological Museum of Amsterdam
}

If during one's work one comes across certain names again and again, names of old collectors, of one's colleagues in earlier days, who with their private museums laid the foundation of our scientific institutions, one naturally becomes keen on knowing more about them. The results of this curiosity, collected by and by in spare time and sometimes too in time stolen from other more serious occupations, are given here to the public in the hope that they may meet their interest. As to me I thought it a pleasure and certainly worth the trouble. I first collected all that I could find in a casual way and at last I hunted for them more systematically, until the time came that circumstances put an end to it.

Incomplete though this list may be, I now send it to the press, hoping that it may give many facts and many sources unknown till now either to historians or to zoologists. When I prepared my biography of Albert Seba I met so many names of old collectors that roused my curiosity that at last I decided to make a list of them, trying to find out what sort of people they were and recording what is known about their collections. It was not easy to put a limit of time to the list, so that finally I included all Dutch collectors I could find up to the present day. If I have omitted some I hope I shall be forgiven, and that readers will provide me with the names of those omitted, that they may serve for a supplement.

First of all I collected all I could find in the rich Library of our Zoological Gardens "Natura Artis Magistra". Miss Joh. Scheffer, Librarian, did all she could to provide me with the continuous stream of sale-catalogues, works on shells, insects, birds etc. etc. which I wanted. Of great help was the advice of $\mathrm{Mr}$ H. VAN DER BIJLL, scientific assistant and of $\mathrm{Mr} \mathrm{H}$. J. Wijnman at the University Library of Amsterdam, where, thanks to the kind help of Mr OosterbaAn and his colleagues, it was possible to look through all the books of travellers who according to JACOBSEN JENSEN visited Amste,dam and the other Dutch cities. In the Library of the "Vereeniging 
ter Bevordering van de Belangen des Boekhandels" at Amsterdam I obtained the very effective help of Miss E. Dronckers, profiting by the beautifully arranged collection of Sale Catalogues in which $I$ found many names. It was a great surprise to me to come across the M. S. Catalogue of sale catalogues of Vosmaer I790, of which I knew the existence, but not the place where it was kept.

I had proceeded with the checking of collections up to 1800 when the beautiful Repertorium of LUGT appeared. Although I had found some catalogues not mentioned in this work I decided to rely on it for information about the sale of collections after 1800 . It would have taken more time than I could spend to consult the ever increasing number of catalogues from I800 up to now. Morover it was certain that the hunting for natural history collections would be less and less succesful, as the interest for such private collections decreases rapidly in the 19-th century. To Mr Lugr my thanks and compliments are due for his publication.

In the Amsterdam Archives, alas the only Archives which I could consult, I met with much kind help and information as usual. Mr. VoorthurJsen acquainted me with a discovery in the "Notarieele Archieven" and with the sale catalogues preserved therein.

I had hoped by reading the older descriptions of the different towns to find out some more private collections or "Cabinets". Interesting though the stuff was, the harvest mostly proved to be nihil. He who is acquainted with this vast literature will understand why time obliged me to stop.

The special attention Dr. MacGillavry gave to my subject, provided me with many new sources. I also had the experienced help of Mr. CorporaAL; and my colleague Kruseman did all he could to complate my list. Prof. Swaen, Miss Beijdals, Miss Mendels, Dr. Eijkman, my colleagues van Seters, van Deinse, Barendrecht they all assisted me in every way.

My colleague, Miss van Benthem Jutring, who studied the history of the Mollusc-collections of our Museum for this Centenary-number used this list, though in statu nascendi. She kindly corrected the proofs during my absence. We could help each other with many an item.

It is thanks to the untiring interest of Professor DE BEAUfort, Director of the Zological Museum, that I could bring this publication to an end!

Last but not least some colleagues from abroad were so kind as to read up some books that were not attainable in Holland, viz. Prof. ARndT in Berlin, Dr Mortensen in Copenhagen, $\mathrm{Mr}$ Winckworth in London and $\mathrm{Mr}$ F. G. Rendall in the British Museum.

In the list of literature given at the end I included only such publications as were often quoted, others were given in the text. Mostly the works of the persons mentioned are not included, if they do not describe the cabinet. They may easily be found in such works as BoeHmer, Cobres, Dryander, Engelmann, Gronovius, or in the biographical papers. If any 
person had no real zoological collection (e.g. Leeuwenhoek) or did not live in Holland (e.g. Bleeker) I omitted his name.

The prefixes van, van Der, TER, DE, do not count in Dutch, so van DER Meulen, must be sought under Meulen etc. Double names have been put under the first name, though mostly the first was only later acquired. $I$ have put the Dutch $i j$ after $i$, but $y$ after $x$.

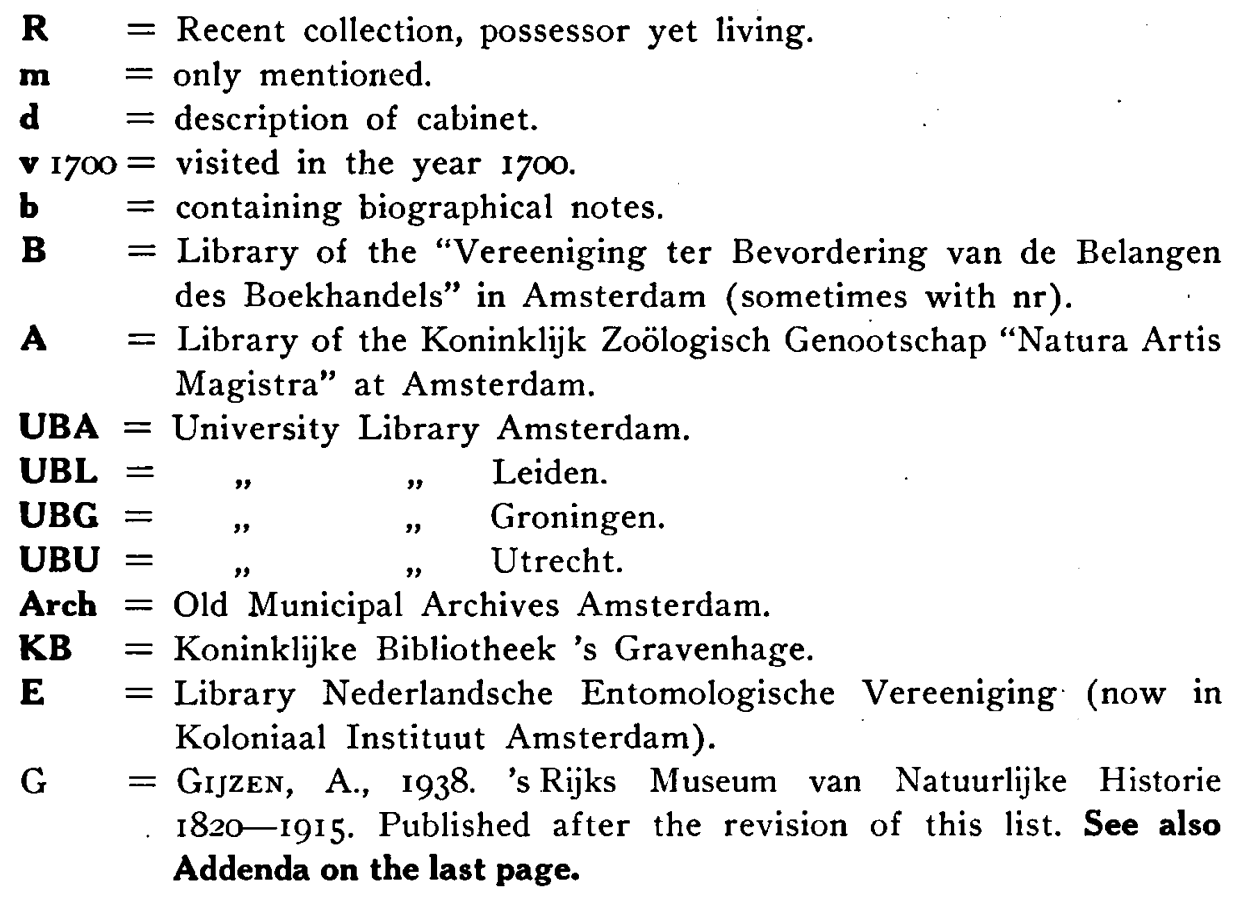

I Aarden, Mrs van, Cabinet sold Utrecht 1775 (Vosmaer I790, m).

2 Aarssen, van, van Hoogerheide, Heer van Voshol etc. etc. Cabinet of shells in the Hague; Valentijn I726 III p. 562 (m); Valentijn I754 p. 46 (m).

3 Acquet, Henricus D', Dr. med., baptised Delft 22. VI. 1632, died I6. VIII. 1706, "Stadsdocter" I67I-I706 "Schepen" and "burgemeester" Delft; Cabinet of shells, insects etc.; Acta erud. Lipsiae I704 p. 55 ("noster d'Acquet"), 363 (m); Jacobaeus I9Io p. 84 (d, v 5. VIII. I674, Hacquet); Uffenbach I754 III p. 339 (v I7Io, Dacquet); Valentinus I7I4 II p. I9 (m, Dacket); Valentijn I726 III p. 56r, 564 (m); id. I754 p. I, 45, 48, 50, 62 (m) ; Kanold I727 p. 195 (m) ; Rumphius I705 preface, p. 3, 4, 6, I0, I I, I3, 16, 19, 32, 36, 37, 40,42,86, 107, 108, 160, $297(\mathrm{~m})$; his collection was sold Delft 29. V. I708 seq. (Acquet \pm I708), Boehmer I785 I p. 386 mentions a catalogue in $\mathrm{f}^{\circ} \mathrm{I708}$, also Vosmaer 1790 . Horn \& Kahle I935-'37 p. I ; Ned. Ts. v. Geneesk. 82, I938 p. I56, 193 . 
4 Admiraal, Jacob L'. I699-I2. IX. I770, painter and “ijkmeester generaal”. Cabinet of insects at Amsterdam. Admiral 1774 (d, b); Houttuijn I76I-'73 vol. 12, p. 29, 86, 206, 242, 444, vol. 13, p. I74-I76 (m); Voet I806 I p. 79 (m); collection sold Amsterdam I77I (Admiral I774 preface, Vosmaer I790, Horn \& Kahle I935-'37 p. 2); van der Aa I852 (b). In UBA a collection of his celebrated anatomical drawings.

5 Aili.y, A. J. D'. Amsterdam Io. VI. 1793-16. I. I85I. Chemist. Insects. Sepp I762-1860, IV, preface, p. 2, 51, 75, 78, 82, 86, 96, I17, I23, I26, I31, I37, 146, 156, V, preface, p. 3, 10, 14, 28, 40, 50, 78, 96, I 13, 136, 139, 152, VI, p. 8I, I32, I50, I72, VII, p. 76, 108, I13, I74, I80 (m); insects bought by Eyndhoven (Eyndhoven I86I); Horn \& Kahle I935-'37. Probably this is J. d'atilly, conservator 's Lands Kabinet v. Nat. Hist. Amsterdam (G. p. 5). (Information of Dr. A. J. d'Ailly Jr.).

6 Aken, C. \& W. van. Travelling menagerie. Witkamp I86I II p. I I8; Rotterd. Dierg. 1858 p. 50; G p. 49, 173.

7 Albarda, J. Herman. $\dagger$ I898. Sepp 1762-1860 VIII, p. III (m); Odonata in Museum Leiden (Lieftinck 1925 p. 66) ; Horn \& Kahle I935-'37 p. 3, 314 (Pal. Neuropt.) ; in Zoological Museum Amsterdam birds collected by him.

8 Alberti, Ds. Ericus Fredericus. Emmerik 1723-Amsterdam 1788. Lutherian clergyman Amsterdam. Cabinet of insects, especially Lepidopt., Scarab.; Sander I783 (d, v I8. VIII. I777); Cramer I779-'82 I p. 5, 21, 34, 4I, 7I, 73, 100, 107, II6, II7, I47, I48, II p. 4, 6, 10, 31, 34, 37, 50, 89, 93, I02, I20, I21, I22, I26, I29-г 31 , III p. 23, 44, 75, 77, 89, I66, IV p. 20, 62, 78, 93, 94, 95, I09, I I0, I16, II7, I22, I25, I26, I75, I77, I 82 , Appendix p. I65, I73 (m); Stoll I7881 p.? (m), I788 2 p. I I9 (m) ; Argenville 1780 p. 355 (m). Molhuysen I9I I--I937 I Kol. 57 (b).

9 Albinus, Bernhardus. Dessau 7.I. 1653-Leiden 7. IX. 1721. I702 Prof. med. Leiden. Seems to have begun the celebrated collections of his son: His library was sold I4. X. I77I. For his life and work see van der Aa I852 (b) ; Molhuysen I9II-I937 IV Kol. 2I (b).

Io Albinus, Bernhardus Siegfried (Bernhard S. Weiss.) Son of preceding. Frankfurt a. O. 24. II. 1697-Leiden 9. IX. I770. stud. Leiden, I7I9 Lector, I72I Professor anat. et med. Cabinet especially anatomy, bought I8. X. I77I for fl. 6000.- - for the University Leiden (Index supellectilis anat.... B. S. Albinus, Lugd. Bat. I77I; after the purchase his brother edited: Supellex anatomica... B. S. Albini, Lugd. Bat. 1775), for the greatest part preserved till now (Geijskes \& van der Klaauw I934 p. 8). His library was sold I4. X. I77I. For his life and work see van der Aa $185^{2}$, Suringar 1866 p. 24,1867 . M. S. of one of his lectures in UBA. He was visited by Haller 1883 p. $37,39,40,42,51,52$, 106-7 (v I725-8); Beckmann I9I2 p. 379, 396 (v I762); Ferrner I9Io p. 482 (v I759). Later on collections mentioned by Rudolphi I804 ( $\mathrm{v}$ I802); Niemeyer I823 (v 1806); Mac Gregor I835 (v I833); Molhuysen I9II-'37 IV Kol. 22 (b). 
I I Alensson, A. Cabinet sold Amsterdam I759 (Vosmaer 1790 m).

I2 Alkman. Natuurhistorisch Museum, Oudegracht 24. Director: W. P. de Vries, founded \pm 1936. $\mathbf{R}$.

13 Allamand (non Allemand), Johannes Nicolaas Sebastiaan. Lausanne 18. IX. I716 - Leiden 2. III. 1787. Prof. Philos. Franeker 3. III. 1747, Prof. Philos. et Math. Leiden 30. V. 1749. Cared for the cabinet of natural curiosities of the University Leiden (in the gallery of the Hortus, see "Leiden") and bequeathed his own important collection to it (now in Museum Leiden). Bocage I764 III p. 89 (v I750); Ferrner I9Io p. 464, 478, 486 (v I759); Beckmann I9I2 p. 388, 37 I (v I762); Courtanvaux I768 p. 204, 208-210 (v I767); Coyer I775 p. 273-4 (v I769); Grimm I775 p. 301, 302 (v I774); Björnståhl I782 V p. 385, 424 (v I775); Sander I783 p. 5I9 (v I776); Honoré I779 p. I43 (v I776); Ehrhart I783 p. 245 (v I783); Smith I796 p. 9 (v I786). Anonymus r767 p. 326 (m); Francq I773 p. 37 (m); Argenville I780 p. 347 (m); Hublard I9Io p. 84 (m); van der Klaauw I926 p. 2, 3 ; $1926_{2}$ p. I89; Suringar 1872 2 p. 266, 270, 27 ; Horn \& Kahle I935-'37 p. 5; van der Aa 1852; Camper I88I p. II (letters); a M. S. of Allemand in UBA (H. S. Catal. II $n^{\circ} \mathbf{r}_{420}$ ). Van der Aa I852 (b); Molhuysen I9II-I937 I Kol. 75 (b). - Catalogue of his books, sold 20. VI. I77r (B).

14 Allemand vide Allamand.

I5 Alphen, Johan van, Dr med. Inspector Collegii Medici. Amsterdam, Keizersgracht near Westermarkt. Catalogue of sale after his death: 8-9. IV. I789. All classes of animals, anatomy. (B $\mathrm{n}^{\circ} 536$ ).

I6 Alpy see Lodewijk Napoleon.

17 Ameshoff, Arnoldus. Amsterdam. Had menagerie on his country residence: "Amstellust", Temminck I808-'9 (m); Temminck \& Laugier I 838 I pl. 426 (m); Witkamp I864 p. 8 , I872 p. X, I888 p. 3I5 (m). He also possessed a cabinet of shells (Argenville 1780 p. $356 \mathrm{~m}$ ).

I 8 Amsterdam. Early after the encircling of the town by stonewalls lions were held by the municipality (Loisel I p. I56 seq.). Swans also were kept (Witkamp I872 p. V). The East Indian Cy had stables for the animals that the ships brought from East and West (Witkamp 1872 p. IX, I875 p. I52). The captains of ships also often showed curious animals to the public in some tavern e. g. De Witte Olyfant, Botermarkt (announcements of the time, about 1700 , in Velten: manis, tapirus; Blankaart, Collectanea Medico-Physica I680 p. 68, elephants I678 and I679; Jacobaeus I9Io p. 27, stuffed rhinoceros, lynx, raccoon, civet-cat, ice-bear I672; Witkamp I872 p. IX, rhinoceros; id. I875 p. I52 elephant, tiger). Many merchants kept a menagerie in their country residence. See also Blauw Jan, and Loisel I9I2 II p. 50 seq., 300, 323, III p. 34. Here he mentions the menagerie of King Louis Napoleon, which under Reinwardt and G. Vrolik shortly thrived in the Hortus Botanicus, spring I809July I7th I8Io, see also Huber I8I I p. I90-i93 (v I809). (On the Hortus 
Botanicus in general see Gedenkboek 1932 p. 56, 264, 393). It was not the first time this garden saw animals within its walls: Haller 1883 p. 102 ( $v$ I 26 ). Like in Leiden a cabinet was preserved here: Sander I783 p. 562 (v I777); Veryard I70I p. 8 (v I682) Willebrandt I76I p. 9I ( \pm I757); Haupt I8I4 I p. 294, II p. 99-IOI (v I810); Terwen 1855 p. 55; and especially Wagenaar I765 p. 387 . Animals in liquor, shells etc. were kept in the Offices of the East and West Indian Cies (Seba 1734-'65 I p. I75, II p. I35; Kanold I727 p. 22 ; Mundy 1925, p. 72, v 1640 an elephant's skull in the West India house; p. 7I shell over the door of the warehouse in the E. India House; Fuchs I9I6 p. 4, Io). See also Moes 1899 p. 5, 8, who mentions the collections of the "Factor von Leipzig" and a house "darinnen allerhandt schöne coquilles" which prof. Barlaeus 1646 showed to the visitor. Perhaps this last is the same collection as that mentioned by Pacichelli 1685 p. 639 (v 1674 ), Kanold 1727 p. 22 and Argenville 1742 p. 215-6, which later on was kept in the "Trippenhuis" as Cabinet du Roi later 's Lands Kabinet van Nat. Hist. (Gedenkboek 1932 p. 275 ; Muntendam 1938 p. 489, under Reinwardt and when he went to East India under Temminck and van Marum, 1815I820) and in 1820 went to the Leiden Museum (G p. 30 a.o.).

Of course the Collegium Medicum (UBA, M. S. I H 37, II $\mathrm{n}^{\circ}$ I 429; Gedenkboek 1932 p. 184,250 ) and the Collegium Chirurgicum (UBA, M. S. II $\mathrm{n}^{\circ}$ 1430, 1431, I C 33; Arch; Gedenkboek 1932 p. 165, I66; Nuyens I928; see also Gedenkboek 1932 p. 284) possessed in their Anatomy-hall (Gedenkboek 1932 p. I78, 407; d'Ailly in Amstelodamum XIII 1926 p. 25 ; Wagenaar 1765 II p. 383,384 ; Veder in Eigen Haard r9I I p. 218, 270, 292, 358, 46I, 565, 764; Amstelodamum X p. VIII-IX 19I2) a cabinet of anatomy and of all sorts of natural curiosities. See: Kanold 1727 p. 22 ; Valentinus I7I4 Appendix XV, p. 52 ; Fuchs I9I6 p. 2, 7-Io; Murray I904 I p. 145 ; Jaarboek Amstelodamum X I9I2 p. VIII-IX; Olivier I838 p. IOI; van der Vijver I848 II p. 9; Calixtus I782 p. 315 (v I65I); Brown I682 p. 22 (v I668); Uffenbach I754 p. 545-548 (v I7IO); Thunberg I792-'4 I p. 63 (v I770); Grimm I775 p. 33I (v I774); Titius I783 p. I90 (v I777); Desjobert I910 p. 206 (v I778); Schaeffer I794 p. I30 (v I788); Rudolphi I804 p. I05-'7 (v I802); Haupt I8I4 I p. 190, II p. IOI (v I8Io); Hills I8I6 p. I4I (v I8I5); Carter I8I9 p. 23I (v I818); Anonymus I8I9 p. I32 (v I8I9); Otto I825 p. 463 (v I822); Goethe und Zelter I9I3 II p. 234 (v I823); Jaeck I826 II p. 234 (v 1824); Duchesne I834 p. 263 (v I834); Horn 1831 I p. 367 (v I830); Mac Gregor I835 I p. 253 ( $(\mathrm{I} 833 \mathrm{~m}$ ); Gunn I834 p. 5I (v I834); Clausade I835 p. 229 (v I834); Czermak I879 I p. I7I (v I850): the present anatomical collections are founded on the collection of $G$. and W. Vrolik (Dusseau 1865 ).

A rarity shop is mentioned by Uffenbach I754 II p. 4I7; bird shops may be found in Nierop 1932 p. I80 (Cleunenburg); p. I9I (van der 
Hoff); p. 204 (Rooseboom). Likewise the yearly fair was an occasion to see curious animals living and dead (A. Fokke, De Amsterdamsche kermis. I801 Amsterdam, p. 32 ; Lax I838 p. I03, I22) and from a travelling menagerie the Amsterdam Zoological Gardens "Natura Artis Magistra", founded 1838 , derived its animals, as will be described more detailed elsewhere in this number. The first zoological collection of the garden, even the first beginning of it at all, consisted in a collection of stuffed animals, especially birds, formed by $R$. Draak on the garret of the Municipal Orphanage, Kalverstraat, exhibited 1837 in the New Town Inn in the Plantage (Dethmar . $838-4$ I III p. 76), later 1838 Oct. I5 in the garden of the Zoological Society of which Draak became the first Director until 28. II. I840, he disappeared with his collections, disagreeing with the managers. Then G. F. Westerman (I807-I890) became director and at once a new collection was started. In the guides of the Garden, in the Archives of the Garden, in the Jaarboekjes published 1852-1875, in the paper on our shell-collections by Miss van Benthem Jutting in this number, in Witkamp 1872 p. XIII, p. XIX, I864 p. 24; Maitland I863 p. XLIX, I888; Leeskabinet I845 III p. I96, IV p. I ; Kerbert I898 p. I8 seq., and especially in Kerbert 1926 and Gedenkboek 1932 p. 509, 513 seq. much information will be found. The present status of the Museum, since 1892 combined with the Zoological Museum of the Amsterdam University will be found in Musea 1938 p. $78 \mathrm{n}^{\circ} 33$. Some literature on Garden, Aquarium and Museum may be given yet: Amsterdamsch Jaarboekje I889 p. 4I; Guislain I842 p. 92 (v I84I); van der Vijver I848 III p. I34; Terwen I855 p. 39, 54; Kalff I875 I p. I52-I66; Czermak I879 p. I7I (v I850); Horn und Kahle I935-'37 p. 187, 314; Loisel I907 p. 98, 1912 III p. 202, 209, 358; Kerbert 1888, I905, I906 etc. etc. (see elsewhere in this number).

The anatomo-zoological collections of the University were begun 1877 by W. Berlin (1825-1902), who since 1864 was Prof. Anat., but I877, when the Athenaeum became University, Prof. Zool. (Gedenkboek 1932 p. 273-28I). At once the connections with the Zoological Gardens started. Under Max Weber (1852-1937) the separation of anatomical and taxonomical collections was effected. In 1923 the first got a new building in the Zoological Gardens, the second remained in the Aquarium but their building was renewed (Gedenkboek 1932 p. 509-517).

Other collections containing zoology can be found in Musea 1938: Koloniaal Instituut p. 42, $\mathrm{n}^{\circ}$ I6 (see also Gedenkboek I932 p. 519); de Pinksterblom p. $49, \mathrm{n}^{\circ} 22$; de Tonijn p. $73 \mathrm{n}^{\circ} 29$. Moreover the zoological possessions of the Dutch Central Institute for Brain Research (Gedenkboek 1932 p. 523; C. U. Ariens Kappers, in: Methods and Problems of Medical Education Ioth series, Rockefeller Foundation 1928) may be mentioned. 
See also s.v. Blauw Jan, Keizerkroon etc.

19 Anderson. Probably the Hague, shells, bought by Lyonet (Hublard I9ro p. 87). Vosmaer I790 (sold I76I). Argenville I780 p. 342 (m).

\section{ANONYMI \\ $\mathbf{L}=$ Lugt $1928 \mathrm{n}^{\circ} \ldots$; Vo = Vosmaer 1790 under "Onbekend".}

Shells from Ambon see Vosmaer 1800 p. $10 \mathrm{n}^{\circ}$ I89; 20. III. I726, L 345 ; la Haye?, 29. X. I728, L 376; Delft I738, Vo; 's Gravenhage r738, Vo (2); 's Gravenhage I739, Vo; Rotterdam I740, Vo; Amsterdam I744, Vo; 's Gravenhage 1745 , Vo; id. 24. V. I746, B n ${ }^{\circ} 177 ;$ Middelburg 23-24. VII. I749, B n० 196; Amsterdam 1750, Vo (2); id. I751; id. 4. XII. 1752, L 799; id. I754, Vo; id. 1755, Vo; id. 1757, Vo; id. I758, Vo; id. I759 (2), Vo; Utrecht I760, Vo; 's Gravenhage I760, Vo; Amsterdam I760, Vo (2); ? I6. XII. 1760, L I124; Amsterdam I76I, Vo (3); id. I7..., Vo; Haarlem 1762, Vo; Amsterdam 28. VII. 1762, L 1233; id. I762, Vo; id. I763, Vo (2); id. 24. I. I763, L I26I ; id. I764, Vo; id. I9. XI. I764, L I4II ; 's Gravenhage I764, Vo; 's Hertogenbosch 7-Io. II. I764, B n' 306 App. p. 47 ; ? I4. I. I765, B n ${ }^{\circ} 314$ p. I59; Amsterdam, I4. III. I765, L 1429; 's Gravenhage 20. V. I765, A $\mathrm{n}^{\circ}$ 104; Middelburg 19. VI. $1765, \mathbf{B} \mathrm{n}^{\circ}$ 319; Rotterdam, 19. XI. I765, L I484; Amsterdam I765, Vo (4); id. 1766 (3) ; Utrecht I766, Vo; Amsterdam I767, Vo; 's Gravenhage 25. V. 1767 App. p. 45, B n ${ }^{\circ} 337$; Middelburg 3. IX. I767, B n ${ }^{\circ} 341$ p. 29; 's Gravenhage I3. VI. I768, L I69I ; id. I768, vide Cobres I p. I4I ; Amsterdam 1768, Vo; id. 30. III. 1769, L I747; id. 5-6. VII. I769, L I77I ; id. I6. V. I770, L I846; id. I770, Vo (2); Haarlem I771, Vo; Amsterdam 1772, Vo; 's Gravenhage 1773, Vo; id. 25-27. XI. I773, L 2205; Amsterdam 9. XI. I773, vide Dryander Banks I p. 225; Haarlem 9. VIII. 1774, L 23I7; Amsterdam 1774, Vo; Leiden, 16. X. I776, B n 422 ; id. I776, Vo (2); Amsterdam 7777 , Vo; id. 1778 (2); Haarlem 8-9. IX. I778, L 2889; Amsterdam 27. V. 1779, L 3006; id. I779, Vo; id. I780, Vo; 's Gravenhage 1781, Vo; Leiden 1783, Vo; Amsterdam 1784, Vo; Leiden 1790, Vo; 's Gravenhage I790, Vo; Rotterdam 12. IX. I791, L 4787; Amsterdam I6.IV.I792, L 4900; 's Hertogenbosch 23.IX.I793, B n 605 p. 94 ; Amsterdam 9. V. I794 II p. 8, B n ${ }^{\circ} 623$ II ; 's Gravenhage 27. X. I794, B n 630 p. I34; id. I4-24. XI. I796, L 5509; Amsterdam I798?, L 5842; id. 20. VI. 1803, L 6657 ; id. $28-30$. IX. I807, L 7301 ; id. 12-I3. VI. I8I I, A n ${ }^{\circ} 3252$; Haarlem II. III. I8I6, L 883I; id.; 24-27. IX I8I6, L 897I; Amsterdam 9. III. I819, L 9530; id. 22. VII. 1823, L I0499; Rotterdam 23. XI. I825, L 10998.

20 Anslijn, Nicolaas, Nic.zn. Leiden 12. V. 1777 - Haarlem I2. IX. I838. Teacher. Van der Aa I852 I (b); Molhuijsen I9I I-'37 I p. I55 (b); Ekama 1874 I p. $103 \mathrm{n}^{\circ} 482$ (cabinet sold 5. XI. 1839), II $\mathrm{n}^{\circ} 882$.

2 I Arnhem. Museum Nederlandsche Heide Maatschappij (founded I9I Utrecht, since I9I4 Arnhem). Musea I938 p. 82, n $38 . \mathbf{R}$. 
22 Arnould, mistake of Argenville I780 p. 338 for Arnould Gevers.

23 Assen seems to have had a zoological garden, see prospectus of the periodical "Fauna" Amsterdam, I882, n' I (A).

24 Assendelft, Mr Dankert van. 's Gravenhage 21. III. 1698-27. X. 1752 Postmaster and Master of the Orphans. Lugt $1938 \mathrm{n}^{\circ} .1246$ (cabinet of nat. hist. and shells sold 19-20. X. I762). Molhuijsen I9I I-'37 VII Kol. 30:

25 Assendelft, W. van, buried I4. III. I744 's Gravenhage. Cabinet of shells sold 1744 (Vosmaer I790). Molhuysen I9I I-'37, VII Kol. 43.

26 BaAN. Haarlem. (Argenville I757 p. 149).

27 BaARs. Haarlem. Birds, insects, some shells. Valentijn i726 III p. 562.

28 BaArt, Willem. Baptised Haarlem I3. VI. 1696 - buried Haarlem 30. V. 1761. Was 1719-1761 notary in Haarlem, procurator 1719-1728, "Secretaris van de Hooge Vierschaar en Bailluage van Kennemerland". Catalogue of cabinet of shells and zoophytes, sold after his death in Amsterdam 13. IV. 1762, seq. (A n ${ }^{\circ} 3244$, also mentioned by Vosmaer 1790 and Lugt $1938 \mathrm{n}^{\circ}$ I216). Argenville 1780 p. 356 (m); Murray 1904 II p. 96. See also Catalogue Leers 1767 p. IX.

29 BACKER, Willem Corneliszoon. Cabinet of all sorts of natural curiosities sold II. IV. I73I in Amsterdam. Catalogue in Arch. (Lugt $1938 \mathrm{n}^{\circ}$ 407).

30 BACKer had a menagerie near 's Gravenhage (Loisel I9I2 II, p. I50; Temminck, Hist. Nat. des Pigeons et des Gallin. exot.).

3 I Backer, J., Senior and Junior, Arnhem. Sepp I762-I860 IV p. I72 (m); Horn \& Kahle 1935-'37 p. 9. (Dutch Lepidoptera to J. Th. Oudemans).

32 BARD. 's Gravenhage, Uffenbach 1754 III p. 366, 390 (v i7Io), cf. Bart.

33 BARDT mentioned in Velten \pm 1700 possessing a sheep from Arabia.

34 Barendrecht, Dr Gerard. Born 1906. Conservator for entomology, Zoological Laboratory Amsterdam, since 1938, collects Hymenoptera. R.

35 Barnaert or Barnaerts, Jacobus. Haarlem, 6. X. 1727-2. XI. I780. Cabinet of birds (Francq I773 p. 36) sold 1781 (Vosmaer 1790, who writes Barnart, I).

36 Barnart see Barnaert.

37 BART, Jacob, died 1725. Cabinet of shells (Valentijn I726 III p. 562, 572, I754 p. 46,56 ). This is probably Bard mentioned by Uffenbach 1754 III p. 366,390 in the Hague.

38 BAS, DE. Notary in 's Gravenhage, kept birds (Loisel I907 p. I I5, I9I2 III p. 87).

39 BASter, Job, Dr Med. Zierikzee, 2. IV. I709-6. III. 1775. Biography in: de Kanter, in: De Vriend des Vaderlands 1831, V 2, p. I25; Nagtglas, J. Baster, Middelburg I875; Nagtglas I888-'93 I p. 23; de Vos, De Vroedschap van Zierikzee, Middelburg 1931 p. 207, 213; van der Aa I852-I878 I; Molhuysen I9II-I937 VI Kol. 74 (b); Collot d'Escury 
I844 VII p. 239; Fokker \& de Man I9or p. 5, 6; Stöver, Leben des Ritters Carl von Linné I 1792 p. 380 ; Kerbert I888 p. 6; Maitland I855 p. I67; Houttuijn $1761-73$, I7 p. 309. He is well known for his investigations on the marine animals near Zierikzee (Opuscula subseciva $1759-' 65=$ Natuurkundige uitspanningen, see also Philos. Trans. R. Soc. London, Acta phys.-med., Werken Holl. Mij. Haarlem, Verh. Zeeuwsch Gen. etc.). Letters to P. Camper in Library Holl. Mij. Bev. Geneesk. in UBA (Camper I88I p. II). On his cabinet see Bibl. Basteriana (B $\left.n^{\circ} 4^{1} 15\right)$ p. 80, 82, and Gedenkboek Zeeuwsch Genootschap I9I9 p. I04 (his widow gave his side-board made of shells to the Zeeuwsch Genootschap).

Bataafsch Genootschap see Rotterdam.

4 I BaÜrt, this is Jhr. P. Bout, see there.

BAyet, Miss. Uffenbach I754 III p. 388: „eine Verwandtin und gewesene Haushalterin von Herrn Resnerus". See Resnerus.

43 Been, Pieter, florist, Haarlem, had a cabinet of insects (Nemnich 1809 I p. $370, \mathbf{v}$ i 808 ).

Beyerinck, Dr. W., in Wijster (Drente), Biological Station, makes collections for comparison. $\mathbf{R}$.

45 BEKE, Leendert VAN, cabinet of natural history, sold 20. II. I708 (Lugt $1938 n^{\circ} 209$ ).

46 Bels, P. J. Collects ectoparasites and Formicidae. R.

47 Bennetr, Prof. Dr Jan Arnout. Breda 20. XII. I758 - Leiden 9. IX. I828. Was some time physician in Amsterdam, but being of a weak constitution devoted himself wholly to science (in 1815 he was appointed professor in Leiden but never gave lessons and resigned Febr. I8I7). Anonymus I8I9 p. Io0 (v I8I9). Van der Aa I852-'78 II (b). Molhuijsen I9II-I937 II Kol. I22 (b).

$4^{8}$ Bentinck, Hans Willem. Baron, Earl of Portland, Schoonheeten 3. III. I65I - Bulstrode (Buckinghamshire) 4. XI. I709. Had a great aviary on his country seat Sorgvliet near 's Gravenhage. Mountague 1696 p. 45 (v I695); Witkamp I875 p. I5I; Loisel I9I2 II p. 50; Molhuijsen I9I I-I937 I Kol. 299 (b).

49 Bentinck, Willem. Graaf, Heer van Rhoon etc. 7. XI. I704-i3. X. 1773, son of the preceding, continued his father's aviary. Réaumur's cabinet contained many exotic birds that once lived there (Houttuijn I76I-'73, 4, p. $242,252,254,262,367,420$, Streseman 1923 p. 120). Courtanvaux I768 p. 264. Molhuijsen I9I I-I937 I Kol. 30I (b).

50 Bentinck, Ir. G. A. Graaf. Overveen. Cabinet of Lepidoptera (Lempke I936 p. 239, Everts 1922 p. XII). R.

51 Berchmans, Br. see Steyl.

52 Bergh, P. J. van den, L.zoon. 1873-I930, Tilburg later Velp. Macrolepidoptera from East Indies (now in Mus. Amsterdam). Everts 1922 p. XII, Horn \& Kahle 1935-'37 p. 286. 
53 Berghe, Adam DE. "Commissaris van de Schuijt op Delft, op de Leijdsche Poort te Rotterdam". Uffenbach I754 III p. 335 (v d I7IO).

54 Bergmeyer see Blauw Jan.

55 Berk, L. H. van. Driehuis. Shells. R.

56 BERKhey see Francq.

57 Bernard. Merchant in Haarlem. Cabinet of birds. Argenville r780 p. 357.

58 Bernet Kempers, K. J. W., "Oud-Directeur der Registratie en Domeinen". Coleoptera. Everts I922 p. XII. R.

59 Besseling, A. J. Hydracarids. R.

6o Beudeker, Christoffel. Amsterdam born about 1675, buried 24. XII. 1756. Merchant. (W. F. H. Oldewelt, Beudeker's "Oudheden van Amstelredam" in Algemeen Handelsblad Zaterdag I Augustus 1936, Ochtendblad II p. 3, b. According to this important biography his cabinet of shells, coins etc. etc. had to be sold within months after his death, which occurred I756. Vosmaer I790 mentions the sale of a cabinet Beudeker "about" I750). Valentijn I726 III p. 56r (also under van Sugtelen whose cabinet seems to have passed into Beudeker's hands). Kalff I928 p. 207. Van der Aa 1852-'78 mixed up two Beudekers.

61 Beverland, Adrianus. Middelburg 165I-England about 1712. Cabinet of Shells. Nagtglas 1888-'93 I p. 36 (b); Molhuijsen 19I I-'37 VII. Kol. 126 (b).

62 Bevoordt, Cornelis van. Amsterdam. Sale-catalogue 27. V. I799 seq. after his death. Shells, anatomy etc. In Library Dautzenberg 50976 (now Musée R. d'Hist. Nat. Bruxelles).

63 Binguebanc. Amsterdam. Cabinet of Natural History. Haupt I8I4 I p. 337 (v I8Io m). Witkamp I864 p. 8 (m).

64 Bisschop, Jan, Rotterdam born about 1680 , baptised (Doopsgezind) I699, died 5. III. I77I (Wiersum in: Rotterdamsch Jaarboekje 19ro p. 50 and in Oud Holland XXVIII. 3. I910. b. Compare also UBA M. S. Cat. II p. I78, I90). With his brother Pieter (about II years his younger, $\dagger$ I 758) he was a merchant of yarn. Both were unmarried and both made collections of paintings, curiosities, shells etc. and for the purpose hired a second house I743. Argenville I757 p. I49, I780 p. 338 (m); Hublard I910 p. 84; Beckmann I9ı2 p. 359 (v I762); Garampi I899 p. 203 (v I764); Courtanvaux I768 p. I68-I70 (v I767); Riemsdijk, in: Oud Holland X 1892 p. 219 (v I766 of Mnizech); Heinecken I769 p. 66 (v I768 mentions only paintings); Coyer I775 p. 269 ( $v$ I769 only interested in pictures); Honoré I779 p. 27 (cabinet sold). According to Wiersum 1. c. there were catalogues I77I of the books, of the pictures, of the porcelain. The paintings and the shells went to Hope. Molhuijsen I9I I-'37 II Kol. I69 (b).

65 Bisschop van Tuinen, Klaas. I840-igo5 Coleoptera (now Mac Gillavry), Lepidoptera (1905 Oudemans, now Mus. Amsterdam). Horn \& Kahle 1935-37, p. 20, 321. 
66 Bisseling, G. A. L. 's Gravenhage. Dutch birds. R.

67 Blanuw, F. E. † I7. I. 1936 (75 years old). Gooilust, 's Graveland. Wellknown menagerie (after his death to Duke of Bedford?) Founded 1886. Loisel 1912 III p. 87, 1907 p. II5. Zool. Garten XLVII p. 6.

68 BlaAuw or Blaeu (Bleau). Secretary Amsterdam. Cabinet of shells. Valentijn 1726 III p. 56r, 1754 p. 45, 48; Valentinus I7I4 II p. I9; Rumphius 1705 p. 108, I16, I19, 160; Uffenbach 1754 III p. 689 (v 1710); Kanold 1727 p. 182.

69 BlaeU zie Blaauw.

70 Blankant, Steven. Med. Dr Middelburg 24.X.1650 - Amsterdam 23. II. 1702. Probably when writing his: Schouburg der Rupsen, Wormen, Maden en vliegende Dierkens (Amsterdam I688) he had a cabinet of insects. De la Rue I734 p. I2 (b); Van der Aa I852-I878 II (b); Nagtglas.I888-'93 I p. 42 (b) ; Fokker \& De Man I9or, p. 12 (b). Molhuijsen I9I I-'37 IV Kol. I56 (b).

71. Blauw Jan. Under this name was known during the 18 th century (till about 1784) a tavern on the Kloveniersburgwal Amsterdam (now Gebouw v. d. Werkende Stand), where (under different owners) all sorts of living animals from foreign countries could be admired, likewise misformed men and other curiosities. The Library $\mathbf{A}$ contains a MS by Velten \pm 1700 with pictures of the animals shown there and elsewhere. He mentions as the first owner Jan Westerhof (Westehoft) or Jan Blauw or Blauw Jan. Later on the owner was Bergmeyer (Bergmeier). An old engraving of the menagerie by Fritzsch in 1751 exists in two different editions and is often reproduced (Witkamp 1875, I888, Loisel 1912, Kerbert 1916) another etching by Moucheron is mentioned by Meyer 1889 . Animals were ordered here for the menageries at Versailles and Schönbrunn. Van der Vijver I848 II p. 443; Witkamp I864 p. 7, I872 p. X, I875, p. I5I, I888 p. 313; Meyer I889 p. 4I ; Kalff I875 p. I52; Loisel I9I2 II p. 52; Kerbert 1916; Leeskabinet for 1845 , vol. III p. 197 seq.

The scientists of the time visited him, see: Camper, Oeuvres I p. 60 , 75; Allamand in his Dutch edition of Buffon; Pallas 1767-'77 II p. 19, XI p. 84; Boddaert I770 V p. 27 ; Shaw I709 p. 37 (v I700); Oliver I703 p. I409 d; Biema I910 p. 87 (v 1736); Hude I784 p. 225 (v 1755); Willebrandt 1761 p. 91 (v 1757); Ferrner I910 p. 332 (v 1759); Beckmann I912 p. 329 (v I762); Thunberg 1792-4 I p. 63 (v I77I); Björnståhl I782 p. 470 (v I774); Feller I823 II p. 242 (Blaü en Joha v I775 d); Sander I783 I p. 555 (v I776 d); Honoré I779 p. 252 (v 1776); Desjobert I9ıo p. 204 (v I778 d); Merck I9II p. 225 (v I784); Tischbein I861 p. II9 (v 1772 -'3 d).

72 Bleau see Blaauw.

73 Bleuland, Jan. Gouda 20. VIII. 1756-Utrecht 8. XI. I838. Prof. med. Harderwijk, Utrecht (I795). His anatomical collections were well known. 
Album Stud. Acad. Rheno-Trajectinae 1886; Maitland 1855, p. 167; Witkamp I888 p. 306 ; Descriptio musei anatomici quod... Acad. Rh. Traj. concessit J. Bleuland 1826; Meiszner I8I9 p. 52 (v 18I7); Anonymus 1819 p. 209 (v I8I9); Fliedner I83I II p. 4I8 (v I823); Horn I83I p. 383, 377 (m). Van der Aa 1852-I878 (b); Molhuijsen I9I I-'37 I Kol 370 (b).

Blijenburgh, Cornelis van. Amsterdam. Cabinet of shells. See a poem "Strande" by Ph. van Borsselen I6I4 (De dichtwerken van Ph. v. Borsselen door P. E. Muller. Groningen 1937).

75 BLock, Cabinet in 's Gravenhage. Uffenbach I754 III p. 393.

76 Blozm. "Casteleyn op de Admiraliteytswerf te Rotterdam". Cabinet of shells. Valentijn i726 III p. 562 (d p. 572-'73), I754 p. 46, 56, 65 .

77 Blok, R. Cabinet of divers naturalia sold Amsterdam I776 (Vosmaer I790).

78 Blomhoff see Cock Blomhoff.

79 Boddaert, Pieter. Med. Dr. Born Middelburg "Oud-Raad der Stad Vlissingen". Later on he lived in Utrecht where he was Lecturer of Natural History. Translated Pallas and others and described animals from Schlosser's Museum (MS in A). He possessed a large cabinet himself : Pallas I767-'77 VIII, p. I I, X, p. 34; Cramer I779-82 I p. 98, 103, II p. 44, III p. I07; van Phelsum I774 p. 59. His books were sold I3. VI. I796 in Utrecht (UBA 393 E 15 and B n ${ }^{\circ} 640$ ); Van der Aa 1852-'78 II (b); Nagtglas I888-'93 p. 47 (b); Hublard I9Io p. 84 (m); Sander I783 I p. 593 (v I777) ; Argenville I780 p. 336 (p. 337 sub Luchtmans); Björnståhl I782 p. 49I (v I775).

80 BoEr, Matthys DE (Mattias Tebure). Hoogewerff I9I9 p. 73 (v Cosimo de Medici 1667 ). Cabinet of Indian curiosities also natural history, animals, shells.

81 BoerhaAve, Herman. Voorhout 31. XII. I668-Leiden 23. IX. I738. Van der Aa 1852-'78 II (b) ; Molhuijsen r9I I-'37 VI Kol. I27 (b) ; Ned. Tijds. v. Geneesk. I866 (2) II p. I, I99, I9I6 2 n 19 , I9I9 I I ; Janus XV I9Io, XVII 1912 p. I45; Collot d'Escury I844 VII p. 289, 292 ; Van Capelle; Maitland I835 p. 162; Scheltema, Peter de Groote, I p. 136, II p. 79, 229; Uffenbach 1754 III p. 425 ; Haller 1883 p. $37,42,43,51$, 106. Later visitors always mention his grave or his estate, they may be found in Jensen I9I9 under $\mathrm{n}^{\circ} 127,146,163,168,170,173,198,203,207,226,238,252,254,261$, $276,277,290,305$, supplement $28,29,36,44,48,52$ (tomb erected 4 . IX. I762, see maandel. Nederl. Mercuur Oct. I762). More important for us are the collections which were sold after his death I6.XI. I739 seq. (Murray I904 I p. I46 III p. I23; Dryander I798-I800 I p. 224), and partly came in the Collections of the Leiden University ( $v$. d. Klaauw I926 p. I, 46, note 21, 27 ; Siegenbeek I829 II 89) See also Lugt $1938 \mathrm{n}^{\circ}$ 725 (Cat. 19-20. IV. 1750). His books were sold 8 VI 1739 (B $\mathrm{n}^{\circ}{ }_{135}$ ). Often mentioned are the collection of drawings he possessed of South African animals (Boddaert 1770 I p. 6, 8, 12) of which Seba copied many in his Thesaurus. Ned. Ts. v. Geneesk. 1938, 82, p. 147, 175-280. 
82 Boerman, D. J. 's Gravenhage. Mollusks. R.

83 Boers, Adrianus and Pieter. Brothers. "Bailluwen der beide Katwijken en van Voorschoten" acclimatised birds and other animals. Francq I769I8I I III I p. I79, I773 p. 36.

84 Boers, W. S. Sale catalogue 14. VIII. 1794, the Hague mentioned by Hagen I862 p. 6I and Dryander I798-I800 I p. 225.

85 Boers. Stresemann 1923 p. 120 speaks of a collection of birds of Boers in Haserswoude near Leiden.

86 BoERs. According to Vosmaer I790 a cabinet of divers naturalia was sold at Leiden 1782 ("een zekere Boers uit de Indië").

87 Boes, A. G. Born 9. III. I864. Inspector of schools at Ambon (Ig09-II), "wethouder" at Haarlem. Mrs B. Boes-Scharff, his wife, made at Ambon a collection of shells which he gave 1922 to Zool. Mus. Amsterdam. Catalogue M.S.

88 Bogard, van den. Surgeon at Delft. Uffenbach 1754 III p. 337, 345.

89 Bolten, D. Insects. R. Everts 1922 p. XII.

90 Вомме. Leendert. Middelburg 20. II. I727-20. III. I788. Merchant. Well known for his zoological publications in Verhandelingen Zeeuwsch Genootschap (some drawings in A). Molhuysen 191 I-'37 VII kol. I66 (b); Nagtglas I888-'93 I p. 51 (b); Maitland 1855 p. 167; Arch. Notary Angelkot $1738 \mathrm{n}^{\circ} 378$; Kesteloo, Oost Kapelle in Woord en Beeld, Middelburg 1936 p. 93, I09; Gedenkboek Zeeuwsch Genootschap I919 p. 104, I05 on the things he gave to the Genootschap. His own Cabinet was sold Middelburg 6. X. 1788 (Catalogue in $\mathbf{B} \mathrm{n}^{\circ}{ }_{5} \mathrm{I} 6$, minerals, fossils, natural history, shells, birds insects), also mentioned by Vosmaer 1790 ; Boddaert I770 III p. 7 note f.

9r Bonac, Marquis de. Ambassador of France in the Hague where he made a large collection of shells. Catalogue (sold Paris II. XI. I757) in Library Dautzenberg $\mathrm{n}^{\circ} 51978$ now in Mus. d'Hist. Nat. Bruxelles and $\mathbf{A}$; Lamy I930 p. II, 34, J. de Conch. I929 p. 69; Argenville I757 p. 147; Hublard I9IO p. 84 (m) 89 .

92 Bonnac see Bonac.

93 Bonne, W. D., Zwanenburg near Halfweg. Malacology. R.

94 Boomgaert, van Den. Delft. Blainville i743 I p. Io.

95 Boonen, Jan. Utrecht in a M.S. (A $\mathrm{n}^{\circ} 3555$ ).

96 Boot see Bout.

97 Возвоом, Hendrik. Catalogue of insects etc. sold I3-I5 III I760 after his death (B $\mathrm{n}^{\circ}$ 266).

98 Bosch, Henricus DE. Med. Dr Amsterdam. His collections were sold IO. XI. I 777 (B $\mathrm{n}^{\circ}$ 394). Simplicia and anatomy. Pallas I766 p. 227 (Boddaert I768 p. 284). Molhuijsen I9I I-'37 IV kol. 234 (b, the same?).

99 Bout, Jonkheer Pieter. Deputee for Zeeland. 's Gravenhage. Possessed the cabinet of L. Vincent: Argenville 1742 p. 2I 5 (Boot), I78o (Baütt); Vos- 
maer 1790. Cramer 1779-'82 III p. 37, IV p. 67. Hublard r9Io p. 84 (Bautt). Sale-catalogue of his collections 1779 mentioned by Vosmaer I790, Boehmer I785 I p. 408, Cobres 1749 I p. 149. In a copy of Rumphius Amb. Rariteitkamer illuminated by Merian (A) it is mentioned that A. Vosmaer got it from the heirs of Jhr. P. Bout after having catalogued his cabinet (probably for sale).

IOO Boyer, P., Cabinet sold Leiden 1772 (Vosmaer I790).

IOI Braekel, Diederik Louis Baron van Braekel tot den Braekel. Tiel. Collection sold 24. I. 1763 , containing insects and all sort of animals. Catalogue p. 89 ( $\left.\mathrm{B} \mathrm{n}^{\circ} 297\right)$.

102 Braijne see Brayne.

103 Brakel, C. A. van. J. U. Dr et Senator. Haarlem. Cabinet of birds. Nozeman I 1770.

IO4 Brakman, C. Nieuw en Sint Joosland. Born Cadzand 25. V. I879. Head of primary school at Nieuwland (Walcheren) and "Conservator" for Mollusks at the Museum Zeeuwsch Genootschap Middelburg. Collection of mollusks recent and fossil. $\mathbf{R}$.

105 Brandhorst, A. L. 's Gravenhage. Shells R.

106 Brandt, A. van den. † I909. Insects see Horn \& Kahle I935-'37 p. 286.

107. Brandt, Joan Coenraad. Neukirchen in Waldeck 22 XII i703-Amsterdam 26 IV I79I. Druggist Amsterdam (Nierop 1932 p. 177). Sale-Catalogue of his shells 23. X. 1792 (A also in M. S. with his portrait); Murray 1904 II p. I35; Lugt I938 $\mathrm{n}^{\circ} 4956$; Horn \& Kahle I935-'37 p. 29. His shells and some zoophytes are in Zoologisch Museum Amsterdam (see van Benthem Jutting in this nr.); Oltmans in Bijdr. t. d. Dierk. IX I869; Knorr I770 V pl. II, VI, VII, XXVI ; van Phelsum I774 p. 59; Lesser 1756 p. 62; Hublard I910 p. 84 (m); Argenville I780 p. 354; Francq 1773 p. 35 ; Maitland I855 p. I68; Sander I783 I p. 575 (v I776); Titius I783 p. I95 (v I777) ; Eversman I792 p. 25-32, 54 (v I783).

108 Brants, Mr. A. † IO. IX. I93I. He continued the work of Sepp. M. S. and drawings in $\mathbf{E}$.

IO9 Brayne, Joan. Druggist Amsterdam. Sale catalogue 7 VII I693 (Murray I904 II p. I36; Lugt I938 $n^{\circ}$ I53). Boccone I674 p. 36, 278 (v I673). Fuchs 1916 p. 7, 46 (d) ; Jacobaeus I9Io (v 1674).

I 10 BREDA. In this town Kanold I727 p. 23 mentions a Theatrum anatomicum and a Hortus medicus. Sincerus i649 p. 235 saw 16ro a "hippelaphum, equum pedes et caudam cervi habentem", "in atrio arcis".

II BREDA, vaN. Palaeontological collection, partly in British Museum.

II BREDA, Jannetje vAN, wife to L. Vincent, see there.

113 Breda, Anthoni van. Began cabinet in 1674 whichs his brother in law $\mathrm{L}$. Vincent made famous. (Vincent, Wondertooneel der Nature I706 I p. 7, I7).

I 4 Bremekamp, Miss N. E., Bilthoven. Shells. R.

II BREUKelerwaARd see Raye. 
I 16 Brink, F. H. van Den. Mammals. $R$.

I 7 Broek, A. N. Ch. ten. 's Gravenhage. Shells. R.

I 8 Broeke, Berent ten. See Paludanus.

I 19 Broeke, Johannes van den. Cabiret of 36 different animals sold 3. IX. 1767 (B $\mathrm{n}^{\circ} 34 \mathrm{I}$ ).

I 20 Broeke, van den... Hodshon. Amsterdam. Birds (Witkamp I864 p. ro). Beginning I9th century.

12 I Broerse, J. Amsterdam. Coleoptera. Now amanuensis Zool. Museum Am. sterdam. Everts 1922 p. XII. R.

122 Bronkhorst. Painter at Hoorn. Cabinet of Shells. Rumphius I705 p. ro8; Valentijn I726 III p. 562, I754 p. 46.

123 Brouwer, Prof. Dr A. M., Utrecht. Lepidoptera. Lempke 1936 p. 239. R. 124 Brouwerius see Croesen.

125 Brugmans, Prof. Dr Sebald Justinus. Franeker 24. III. 1763-Leiden 22. VII. 18I9. Mr Arts, Dr Philos. (I78I), Dr. Med. (1785), Prof. Phil. Nat. Franeker (1785), Prof. Hist. Nat. Leiden (1786). Possessed himself a cabinet of zoology, anatomy, pathology and did much for the collections of the University. Van der Klaauw I930 p. 49-126, I926 1 p. 2, 5, 6, Io, I 2, 47, 54, 57, 75, I24, I926 2 p. 2 ; Geijskes \& van der Klaauw I934 p. I I ; Horst \& Schepman I908 p. VII; Thouin I84I p. 279 (v I795); Rudolphi I804 p. I27 (v 1802); Niemeyer I823 p. I64 (v I806); Niebuhr I842 p. 78, I34 (v I808-'9); Haupt I8I4 II p. 214 (v I8Io); Meiszner I8I9 p. 73 (v I8I7); Strick I8I8 p. I96 (v I8I7); Collot d'Escury I844 VII p. 268-28I, 332-336; Maitland I855 p. I69; Van der $\mathrm{Aa} 1852-78$ II (b) ; Molhuijsen I9I I-'37 I Kol. 487 (b); G p. 23, 34 a. o.

I26 Bruijn. Amsterdam. Valentinus I7I4 II p. 19; Uffenbach I754 III p. $689(\mathbf{v})$.

I 27 Bruijne, P. DE. Middelburg 5. I. I884. Director steam-navigation-Company on the Schelde. Cabinet of Shells (partly bought from van Rees). $\mathbf{R}$.

128 Buis, Ir Andries Johannes. I860-1936. Bilthoven. Insects. Lempke I937 p. 245 ; Everts 1922 p. XII ; Horn \& Kahle I935-'37 p. 325.

129 BURG, Johan van DER. Lawyer. 's Gravenhage. Cabinet of Shells. Valentijn I726 III p. 562, I754 p. 19, 46.

130 BURG, Harmannus van DER. Amsterdam. Druggist. Had a Cabinet. Rumphius 1705 p. 86,99 , I08; Valentijn 1726 III p. 56 I ; 1754 p. 45 ; in a M. S.: A $n^{\circ} 3555$; Boccone 1674 p. 278 ( $\left.1673-4\right)$.

131 Burgh, D. G. van der... van Kronenburg. Lugt 1938 n $^{\circ}$ I0727 (Catalogue of cabinet sold 27. IX-r. X Utrecht, especially Shells).

132 BuRG, DEN. In this village (island Texel) is a Museum of Nat. Hist. (Musea 1938 p. $\left.95 \mathrm{n}^{\circ} 53\right) . \mathbf{R}$.

I33 Burman, Prof. Dr Johannes. Amsterdam 26. IV. 1706-20. I. I779. Prof. Bot. Amsterdam. Gedenkboek I932 p. 49, 51, 165, 267, 563 (b) ; Collot d'Escury I844 VII p. 307, 315; Van der Aa 1852-'78 II (b) ; Ferrner I9Io p. 359, 
$363,386,387,388$ (v 1759); Thunberg I792-'4 I p. 13, 15, 20, 22, 63, 65, 66, 67, II 2 p. 257,259 (v 1770, 1779); Ehrhart 1783 p. 259 (v I782); Sander I783 I p. 562 (v 1776); Argenville I780 p. 354 ; Arch. Notary Roas $n^{\circ} 884$ i fol. I07; UBA M: S. Cat. II n ${ }^{\circ}$ I $35^{2}$ (preface to Plantae Americanae of Plumier); Björnståhl I782 p. 45I, 47I ( I774); Molhuijsen I91 I-'37 IV Kol. 353 (b).

134 Burman, Prof. Dr Nikolaas Laurens, son of preceding. Amsterdam 27. XII. 1733-II. IX. 1793. Prof. Bot. Arnsterdam. His cabinet was well known, plants, insects, shells, zoophytes, corals, fishes etc. Gedenkboek I932 p. 563 (b), 267, 268; Van der Aa I852-'78 II (b); Molhuysen I9I I-'37 IV Kol. 354; Linnaeus, Syst. Nat. ed. X I758 Reg. An. p. I 17, ed. XII I789 I p. 462 ; Houttuyn I76I-'73, 4 p. 4I3, 436, I7 p. I28; Pallas I $766_{1}$ p. 386 , I766 2 I p. 6, 8, I2 ; Boddaert I768 p. 487; Cramer I779-'82 I p. 72, 75, 76, 122, II p. 97, III p. 100, Appendix p. 149, I50; Stoll I788 2 p. 74, I8I3 A p. 30, D p. 7; Thunberg I792-'4 I p. 23 (v I770); Grimm 1775 III p. 330, 344 (v I774); Björnstahl, I782 V p. 45I, 47 I (v I774); Schaeffer I794 p. I30 (v I 788); Forster I868 p. 87 (v 1790).

135 BuUren, van. Leiden. Insect-collection. Argenville I780 p. 348.

136 BuUrt, Ds. Adriaan. Zalt Bommel 27. V. 171 I-Amsterdam 25. XII. 178I. Clergyman in Beek, Ooltgensplaat, Hanau, Amsterdam (where he lived Fluweelen Burgwal opposite Oudekerk). Collection of Nat. Hist., especially birds. Jensen 1919 p. 127 note 7 ; Argenville 1780 p. 356; Björnstahl 1782 V p. 477, 487 (v 1774); Thunberg 1792-'94 II p. 257 (v r779); Nozeman I770 I (m); Van der Aa I852-'78 II (b) ; Molhuijsen I9I I-'37 VII Kol. 254 (b).

I 37 Bynckershoek, Cornelis van. Middelburg 29. V. I673-'s Gravenhage 16. IV. 1743. Lawyer. President High Court. Cabinet of nat. history, zoophytes, shells etc., sold I8. XI. 1743. Catalogue in B $n^{\circ} 158 \mathrm{a}$ (2nd suppl.). Vosmaer r790. Van der Aa 1852-'78 II (b).

C see also $\mathrm{K}$.

138 Calf, Nicolaas. W.-Zaandam 20. VI. I677-4. V. I734. Merchant, travelled much. Scheltema, Peter de Groote, Utrecht 1842 p. II7, I83 (cabinet sold after his death); Rumphius I705 p. 224; Uffenbach I754 III p. 687 (v I7IO); Poellnitz 1734 III p. 32I (v 1732-'3); Van der Aa I852-'78 III (b).

139 Calkoen, Joan. Amsterdam. † I8I4 (Heerengracht, near Heerenstr., $\mathrm{n}^{\circ} 370$ ). Cabinet sold 30. VI. I8I4 (A n ${ }^{\circ}$ 3366) ; Stoll 1813 A p. 73, B p. 28, C p. 29, 30, 3I, 32, 35, 36, 37, 38, 40, D p. 2, 7 ; Temminck I808 I p. IX; Horn \& Kahle I935-'7 p. 326 (insects to Mus. Leiden); G p. 23, 30; Nemnich I809 p. 370 (v I808-'9); Haupt I8I4 p. 337 (v 1810).

140 Calmette. Maastricht. Cabinet of naturalia especially fossils. Argenville I 780 p. 336. 
14I Camper, Prof. Dr Petrus. Leiden Ir. V. I722-'s Gravenhage 7. IV. I789. Prof. Phil. et Med. Franeker (I750), Prof. Med. Amsterdam (I755). From I76I he lived privately near Franeker. Gedenkboek I932 p. 49, 165, I84, 275, 566, portrait p. 412 (b); Van der Aa 1852-'78 III (b); Molhuysen I9I I-I937 I Kol. $55^{2}$ (b) etc.; He possessed a large cabinet, see A. G. Camper, Descr. succ. du Mus. de P. Camper, Amsterdam et la Haye I8I I ( $A n^{\circ}$ IO2); a collection of chirurgical instruments was described I76I (UBA); his letters and manuscripts I88I (Mij. t. Bev. Geneesk. UBA, see also M. S. Cat. II $\mathrm{n}^{\circ}$ 1405); his books were sold partly 3I.V. I790 seq. (B $\mathrm{n}^{\circ}$ 557); his instruments I9. IV. I790 (B n ${ }^{\circ}$ 555); Nuyens I928 p. 4I (portrait); Luc I779 III p. 44I (v I776); Sander I783 I p. 489 (v I776); Merck I9II p. 180, 207-216, 218, 224, 226, 227, 230 (v I784); Schaeffer I794 II p. II3 (v I788); Forster I868 (v I790), I843 p. 88 (v. I778); Rumpelt I802 p. 74 seq. (v 1779); Duchesne I834 p. 268; Mac Gregor I835 p. 252; Dethmar I840 p. I86, 240.

142 Camper, Adriaan Gillis, Amsterdam 31. I. 1759-'s Gravenhage 5. II. 1820. Worked and lived long with his father in Franeker. His cabinet with that of his father Petrus was bought by the King for the University Groningen (Mac Gregor I835 p. 252). Van der Aa 1852-1878 III (b); Molhuijsen 19Ir-'37 I Kol. 549 (b).

I43 Canselaer, P. D. A. Cabinet sold 's Gravenhage i6. V. 1746 (B n 177 ). (Vosmaer I790 gives: 1748 ).

I44 Capellen, Godert Alexander Gerard Philip Baron van Der. Utrecht I5. XII. I778-de Bilt IO. IV. I848. Gouverneur-Generaal of the East-Indies (1816-1826). His cabinet of shells was bought by van Lidth de Jeude (Cat. Mus. Zool. Lidth de Jeude $1856, \mathbf{A ~ n}^{\circ}$ I30, preface) (M. S. Catalogue in A n $\left.{ }^{\circ}{ }_{10} \mathrm{~A}\right)$; Dethmar 1838 '4I IV p. 44 (v I838); Van der Aa $1852-78$ III (b) ; Portrait and biography in : De Indische Verlofganger I930 p. 885. Molhuysen I9I I-'37 I Kol. 569 (b).

145 Capellen,........ Baton van DeR, Heer van Schonauwen-Houten, near Utrecht. Cabinet of naturalia, shells etc. (Argenville r780 p. 337).

146 Caron, J. R. Hilversum. Lepidoptera. R.

147 Cate, ten see Kate, ten

I48 Cattenburg. Amsterdam. Shells. Rumphius I705 p. I08; Valentijn I726 III p. 56I (Kattenburg), I754 p. 45.

149 Cavallier. Amsterdam. Cabinet. Kanold 1727 p. 22 (m).

150 CerfF, I. DE. Cabinet Amsterdam. Sold i768 (m Vosmaer I790).

I 5 I Ceton, J. C. Albergen near Almelo. Lepidoptera. Lempke 1936 p. 239. R.

152 Chais, Charles. Genève 3. I. I70I-'s Gravenhage Oct. I788. "Pasteur Eglise Wallone" 's Gravenhage. Cabinet of shells. Catalogue by Meuschen 22. IV. 1766, sold in Amsterdam (A $n^{\circ} 3126$; Lugt $193^{8} \mathrm{n}^{\circ}{ }_{1526}$; Vosmaer I790); Argenville I757 p. I49, I780 p. 343; Hublard I9Io p. 84 (Chaize). When his books were sold 's Gravenhage in aedibus defuncti 
IO. IV. 1786 the Catalogue (Vosmaer. I790; B $\mathrm{n}^{\circ} 492$ ) contained also a collection of fossils and zoophytes; Molhuysen I9II-'37 VII Kol. 295.

I53 Chatelain, Mrs. J. J...... born Smith (Schmidt). Cabinet of butterflies. Cramer I779-'82 II p. 66, 88, 95, 98, 99, I45, III p. 21, 47, I08, I09, I54, I56, I58, IV p. I, 6I, I8I, 198.

I 54 Chevalier, Nicolas. Utrecht. bapt. Sedan I. VI. I66I - buried Amsterdam Io. X. -I720. A. Huguenot refugee in Holland. Author of the catalogues of the Utrecht Cabinet of rarities 1707. The greater part of Chevalier's own collection was purchased by the Elector Augustinus of Saxony (Dresden) (Murray I904 I p. 37, II p. I76). Van der Aa 1852-'78 III (b); Molhuysen I91 I -'37 VI Kol. 296. (b).

155 Citters, Mr Casper van. Middelburg 22. I. I674-28. IX. 1734. Lawyer, "Raadpensionaris" of Zeeland (I7I8-I734). Nagtglas I888-'93 I p. I2I (b); Van der Aa 1852-'78 III (b); Cabinet (Valentijn I726 III p. 563, I754 p. $47,63,64)$.

156 Citters, Mr Wilhem van. London 7. X. 1685-Middelburg 28. XI. 1758. Brother of preceding. Cabinet Middelburg (Valentijn I726 III p. 563, I754 p. 47, 63, 64); Nagtglas I888-'93 I p. I24 (b); Van der Aa I852-'78 III (b).

I57 Citters see also Kien van Citters.

I 58 Clerco, Jacob DE. Menagerie on his estate Driemondt at Gaasp and Gein. Ferrner I9Io p. 437 (v I759).

159 Cliever, Cornelis DE. Cabinet of naturalia, sold Middelburg 12. VI. I775. (Lugt $1938 \mathrm{n}^{\circ} 2420$. Vosmaer I790).

160 Cliffort, George, J. U. Dr Amsterdam, 7. I. I685-Io. IV. I760. Merchant and burgomaster in Amsterdam. He lived on his estate. "De Hartekamp" between Haarlem and Leiden and was the Maecenas of Linnaeus I735-'8; Linnaeus Hortus Cliffortianus 1737 (menagerie etc.); Ferrner I910 p. 461, 442 (v 1759), 340, 361 ; Björnståhl I782 V p. 442 (v 1774 estate), 434, 457, 478; Hanway I753 II p. 323 (v 1750). According to Vosmaer I790, Clifford's Cabinet of minerals etc. was sold Amsterdam I760. (Lugt 1938 n III8: Nov. 4, I760 seq.); Francq I769-i8i I III p. 180 (menagerie); Lesser I744 p. 49,1756 p. 63.

16 I Clockner see Klöckner.

162 Chusius, Carolus (Charles de l'Ecluse). Arras I8. II. 1524-Leiden 4. IV. 1609. Prof. Bot. Leiden (I 593-1609). In Hamburg. Magaz. III p. 559-'64: Nachricht von Carl Clusii Naturalien-Cabinet. (Dryander I798-I800 I p. 223); Hegenitius I630 p. IOO (v); Hunger in Ned. Ts. v. Geneesk. 70, 2, 1926 (b) and Catalogus Clusius Tentoonstelling Leiden 1926; Cohen, Clusius in Leijden in?; Van der Aa $1852-' 78$ (b) Molhuysen I9I I-'37 IX Kol. I50 (b).

I63 Cock Blomнoff, Jan (Kock Blomhoff). Amsterdam 5. VIII. I779-Amersfoort I3. X. 1853. Resident of Holland in Japan. Cabinet. On his birds 
see Temminck \& Laugier I838 III pl. 470. Maitland 1863 (Ned. Tijdschr: v. Dierk. I p. LVIII) mentions his collections as in the possession of the Amsterdam Zoological Museum. Molhuijsen I9II'-37 I Kol. 374 (b). I64 Coce van Nereynen, D. DE. "Kapitein-Ingenieur" in Deventer. Cabinet of insects. Sepp I762-1860 I 6 p. 28, 32, 60, 74, III I p. 15, 25, 38, 46.

I65 Coenensoon, Adriaen. Scheveningen I5I4- \pm I583. He wrote a book on fishes as early as 1577-80 (M. S. "Visboeck" in KB) and possessed a collection of dried fishes. Molhuijsen 191 I-'37, VI. Kol. 318(!)

166 Colbius. Amsterdam. Museum. Kanold I727 p. 22, I82; Valentinus I7I4 II p. 19; Uffenbach 1754 III p. 689 (v 17Io).

167. Coldewey, H. Twello. Lepidoptera. Lempke 1936 p. 239. R.

168 CoQ. Rotterdam. Cabinet. Argenville i78o p. 338.

169 Corne, Del, Middelburg or Vlissingen. Valentinus I7I4 II p. 19; Kanold I727 p. 215 (205 Middelburg); Monconys 1665 I p.... (Vlissingen, shells, zoophytes, del Corne was old then, $v$ about 1663 ).

I70 CorporaAl, Johannes Bastiaan. Born i880. Conservator of Entomology Zoölogisch Museum Amsterdam. Coleoptera. (Cleridae). Everts 1922 p. XII. R.

I7 I Court, Petronella de LA, widow of Adam Oortmans, also known as Mejuffrouw Oortmans. See there.

$172 C_{R A B}, P$. van DER. Once resident at Ambon gave his cabinet of shells to the Rotterdam Zool. Gardens (Jaarbericht Rott. Dierg. II I869-'7I p. 54).

173 Cramer, Pieter. Merchant in Amsterdam. $\dagger$ II 1777 . Well known for his books on butterflies (Cramer I779-'82). Anonymus I767 p. 325 (m); Maitland I855 p. I68 (m); Argenville I780 p. 354 (m); van Phelsum I774 p. 36, 53; Pallas I766 1 p. I45, 321, 382, id. I767-'77 IX p. 27; Boddaert i768 p. XXIV, XX, 306, 397, 423, 48I ; Houttuyn I76I-'73 9 p. $182,207,501,536,537,17$ p. I17; Vosmaer 1790 gives 1777 as the date of sale of his Cabinet of divers naturalia; Horn \& Kahle I935-'7 p. 47 erroneously mention his death in 1779 , and record that his collection partly came to C. van Lennep, from whom it passed to A. J. van Eyndhoven, after whose death it was scattered; Sander i783 I p. 576, v I5 Aug. I777, "Herr Cramer starb im letzten Februar am hitzigen Fieber, ... Sammlung fast aus allen Reichen der Natur ... verkauft, die Insekten aber bekam sein Neffe ... Reuszelaar [sic, i.e. Renselaer]". Björnståhl I782 V p. 450 (v I774); Garampi 1889 p. 197 (v I764); Francq 1773.

174 Cramer, Jacob. Merchant Amsterdam. Cabinet of shells, physical instruments. Ferrner i910 p. 322, 377, 385 ( $v$ i759).

175 Cramer, J. Lépidoptérologue Amsterdam. Cabinet sold 22. VII. I8I I (Lugt $1938 \mathrm{n}^{\circ} 8050$ : birds, insects, shells). Is this the same as the preceding?

I76 CRAmer see also Kramer.

177 Cremers, Rector Jos. Maastricht (see Maastricht) collects Coleoptera and Lepidoptera. $\mathbf{R}$. 
I78 Crena, Philippus. "Commies op 't Comptoir van den Heer Ontvanger der Convoyen en Licenten". Dordrecht. Cabinet of shells. Valentijn I726. III p. 563,578, I 754 p. $5,47,62,64$.

179 Croesen, Zara, wife of D. Brouwerius. Cabinet (Valentijn I726 III p. 560 , I754 p. 44).

180 Cronenberg, Gotth., Druggist. Amsterdam. Only some stones. Uffenbach I754, p. 613 (v I7Io). cf. Croonenburg.

i 8 I Cronenburgh, B., Large cabinet of shells (Lugt $1938 \mathrm{n}^{\circ}$ I203) sold 22. III. I762 seq.

182 Croonenburg, G., Cabinet of minerals sold in the Hague 1744 (Vosmaer 1790). cf. Cronenberg.

183 Cronstrom, Daniel Isaac Baron DE. “Général Major \& Colonel d'un régiment d'Infanterie au service de LL. HH. les Etats Généraux, rer adjudant de S.A.S. Mgr. le Prince d'Orange" Cabinet of shells sold Io. IV. I769, 's Gravenhage. (Catalogue $B n^{\circ} 360$ p. 279).

184 Dacket (Dacquet, d'Acquet) see Acquet.

185 Dalen, Pieter van. Gifted Velten (see Velten) with shells which he had possessed for 25 years and which had inherited from his father.

186 Dalen, Dr Cornelis. Rotterdam, 31. XII. I766--24. X. I852. Physician who had a cabinet of natural history especially insects and shells. The Zoological Gardens Amsterdam bought (Sale catalogue 23. VI. 1853 Rotterdam in $\mathbf{A} \mathbf{n}^{\circ}$ I3) the Coleoptera (Gids voor bezoekers Natura Artis Magistra I869 A; Catal. 1.c. p. I07, for $f$ 2000; MS Catalogue $A \mathbf{n}^{\circ} 346 \mathrm{I}$ ) and part of the shells (Maitland I863 p. LIV; Catal. 1.c.). To Leiden went the greater part of the last (Horst \& Schepman 1908 p. VIII), the Lepidoptera were bought by van Eyndhoven (Eyndhoven 186r) from whom they passed to Verloren; Neill I823 p. 122 (v I8I7). Molhuysen I9I I-'37 V Kol. 123 (b).

187 Dall, E. B. ten. "Procureur voor den Hove van Justitie". Cabinet of shells sold I3-2I. IV. I807, 's Gravenhage (Lugt $1938 \mathrm{n}^{\circ} 7220$ ).

188 Dankert van Assendelft see Assendelft.

189 Decourt, F. C. Dordrecht. Collector of insects. Sepp 1762-1860 IV p. $23,88,94$.

i9o Dedem, F. K. Baron van. Cabinet of birds. R.

191 Deinor. Cabinet. Rotterdam. Kanold I727 p. 86.

192 Deknatel, A., Amsterdam. Cabinet sold i765 (Vosmaer I790).

193 Delafaille see Faille.

194 Delcorne see Corne.

I95 Delft. This town like others possessed an anatomy-theatre. I only mention the items I casually met with. Time did not permit a study in the Delft Archives, nor of the special literature on the town. Ray 1738 I p. 25 (v I663); Jacobaeus I9Io p. 84 ( $v$ I674, saw the anatomy and many 
curious animals in the technicotheca: rhinoceros, tiger, rupicapra, civetcat etc.); Uffenbach 1754 p. 338 saw near the townhall a stuffed eagle on his nest; Brereton 1844 I p. 22 mentions tame storks kept in the town; Honoré I779 p. 44 (v I776) saw the Anatomy and the Hortus medicus. Some natural history in Municipal Museum (Musea 1938, p. 96, no 55). 196 Denekamp. Museum Natura Docet. (Musea 1938 p. $102 \mathbf{n}^{\circ}$ 60). R.

197 Dentzel, Johan Melchior, Amsterdam (Fluweelen Burgwal tusschen Hoogstraat en Stoofsteeg). Cabinet of shells, zoophytes etc. etc. sold 2r. XI. 1809 seq. (Catalogue in E).

198 Desmarets, Daniel. "Ser. Brit. Regis Guilielmi III Aedificiorum Praefectus". Cabinet of natural history, insects etc. sold Leiden 8. VI. I7I6 (B). Valentijn I726 III p. 562, I754 p. 46; Uffenbach I754 p. 373 (v I7Io lived in 's Gravesand, 3 hours from the Hague). See Marets.

I99 Dessort, Henry. Anat.-ethnol. Museum Amsterdam. Guide I857 in UBA.

200 Deventer Koloniaal Landbouwmuseum (Musea 1938 p. $103 \mathrm{n}^{\circ}$ 6I). R.

20 I Diakonoff, A., Amsterdam. Assistant Koloniaal Instituut. Collects Microlepidoptera $\mathbf{R}$.

202 Dieden, van. Utrecht. (Houttuijn I76I-'73, I2 p. I8, 64).

203 Dieren. In this village Stadhouder Willem II had a menagerie, begun 1648 (Witkamp I872 p. IX).

204 Dishoeck, Anthony van. Med. Dr. Vlissingen. I643-I728. Cabinet of shells (Valentijn I726 III p. 563). Letter to him I7I9 by W. van Outhoorn in Archief Zeeuwsch Genootschap I937.

$20 ;$ Dishoeck, Anthony Pieter van. J. U. Dr I709-67 Vlissingen, (his house now Townhall) grandson of the preceding. Portrait in: Nederlandsch Patriciaat I9I7 p. I26. Cabinet sold 30.X. I767 at Amsterdam (ed. Meuschen) (A $\mathrm{n}^{\circ} 3 \mathbf{1 2 6}$; mentioned by Vosmaer 1790 and Lugt 1938 $\mathrm{n}^{\circ} \mathrm{1642)}$.

206 Dixon, C. J. 's Gravenhage. Coleoptera Everts 1922 p. XII. R.

207 Docters van Leeuwen, Prof. Dr W. M. Once Director 's Lands Plantentuin Buitenzorg, now Leersum. Collects galls. R.

208 Does, Arnoldus DE. Bookseller Leiden. Catalogue of sale I8 III 1872 (B 454).

209 Doesburg, Pieter Hendrik van. Born 1892 Amsterdam; East India; now Baarn. Coleoptera to Mac Gillavry. Everts 1922 p. XII; Horn \& Kahle I935-'7, p. 286 . $\mathbf{R}$.

210 Doetinchem. This village has a Museum „de Kelder" (Musea 1938 p. 106 $n^{\circ} 63$ ) containing a.o. animals and fossils. $\mathbf{R}$.

2 I I Doets, C. Hilversum. Microlepidoptera. R.

212 Doeveren, Prof. Dr Wolther (= Wouter or Gualtherus) van. Philippine I6. XI. I730-Leiden 31. XII. I783. Prof. Med. Groningen (1754-I77I), Leiden ( $177 \mathrm{I}-\mathrm{I} 783$ ). In his private museum natural history and especially fossils were found. Van der Klaauw 1926 p. I, 47 note 26; Geijskes \& 
van der Klaauw I934 p. Io; Argenville I780 p. 348; Collection sold Leiden i785 (Vosmaer I790, Murray I904 II p. 208, Engelman I86I p. 9). Björnståhl I782 p. 423 (v I775); Honoré I779 p. I42 (v I776); Mac Gregor I835 p. 168 (v 1833 ). Van der Aa $1852-' 78$ III (b) (see also Camper I88I p. I3; and UBA, M. S. Cat. II n ${ }^{\circ}$ 1370); Molhuysen I9I I-'37 IV Kol. 5 I I (b).

213 Domisse, Jacoba. Cabinet sold Middelburg 3I. III. I773 (Lugt' 1938 n $^{\circ}$ 2I 5 I). 214 Doorman, G. Wassenaar. Coleoptera. Everts 1922 p. XII.R.

215 Doorne, M. J. van. Butterflies, sold I. III. I762 's Gravenhage (B n 289). 216 Dordrecht. In this town the first Malacological Society we know of ( 1720$)$ is mentioned by Valentijn 1726 III p. 563. It was called "de Liefhebbers van Neptunus Cabinet". I have already pointed to it in Basteria II 4 I937 p. 64. Argenville I742 p. 2 I6.

217 Doublet, Delft. Witkamp I864 II p. 8, Uffenbach I754 III p. 36I (v i710), Haupt I8I4 p. 337 (v I8Io, so this must be a later generation!)

218 Douglas. Valentijn i754 p. 63,65 .

219 DraAk, Reindert. Cabinet of birds, fishes etc. pupil of Temminck. Attendant at the Amsterdam Orphanage. Collection on the garret of this institution, later in the Town Inn in the Plantage (Dethmar I838-'4I. III p. 76). It formed the first Museum of the Zoological Gardens, Amsterdam, of which Draak became the first Director (Oct. I5. I838-Feb. 28. I840). Witkamp I864 II p. 107; Maitland I888; Lidth de Jeude 1856 preface (bought Draak's collection); Kerbert 1926.

220 Drenth, J. H., 's Gravenhage. Shells. R.

22 I Driessen, Mr. Robertus Keuchenius. Groningen 7. V. 1759-25. X. I831. Lawyer Groningen, Secretary of Ommelanden. Fossils, Butterflies. Sepp I762-I860 III p. 78, 86; Molhuysen I9II-'37 IV Kol. 531 (b). (Collection to University Groningen).

222 Driessen, Prof. Di Petrus. Groningen 30. VIII. I753-II. I. I8ะ8. Prof. Med. et Bot. Groningen ( $1787-1823$ ). Catalogue of his cabinet of animals, fossils, etc. sold I9. I. I829 Groningen [UBA]; Molhuijsen I9I I-I937 IV. Kol. 530 (b).

223 Duymaer van Twist, $\mathrm{Mr}$ Albertus. I809-1887 Gouverneur Generaal of East India $185 \mathrm{I}-1856$. Collected shells. His collection was given by the heirs to the Amsterdam Zoological Museum in 1930 (M. S. Catalogue in A). Kalff in: Indische Verlofganger 1926-'7 p. 568 (b) and in: Onze stem I927 p. 907 (b); Molhuijsen I9II-I937 II Kol. I459 (b).

224 Dulfer, The Rotterdam-Museum got his butterflies. Lempke 1937 p. 245 . $R$.

225 Du Tour see Tour.

226 ECLUSE see Clusius.

227 Eeteren, van probably: van Heeteren. 
228 EijK, Ds. Jan van. Amsterdam 7. VIII.I757-Loosduinen 7. II. I822. Clergyman Loosduinen. Cabinet sold 7. VI. 1822 (Lugt $1938 \mathrm{n}^{\circ}$ I0270). Molhuijsen I9II-I937 VIII Kol. 517 (b).

229 EIJK, J. van. 's Gravenhage. Cabinet sold 18-24. XII. I822 (Lugt 1938 $n^{\circ}$ ro360).

230 Eijkman, Dr. Christiaan, born 9. VI. r882. Dordrecht. Dutch birds and mammals. (Duplicates in Museum Rotterdam). $\mathbf{R}$.

23 I EIJL, A. van. 's Gravenhage. Kanold 1727 p. 198, 306.

232 Eijndhoven, Mr. A. J. van. Amsterdam later Zutphen. Cabinet of insects sold I4. X. I86I seq. in Rotterdam (A). His collection contained according to said catalogue: coll. A. J. d'Ailly, Amsterdam (bought by Verloren for $f 460$ ), coll. Dr. C. Dalen, Rotterdam, (also bought by Verloren for $f(800$ ), coll. van Walcheren, Brummen, (bought partly by Verloren for $f$ II70, partly by Deyrolle, Paris for $f 620$ ), coll. Eyndhoven (bought by Deyrolle for $f 300$ ), coll. van Lennep. (Eyndhoven 1861 ). Horn \& Kahle I935-'7 p. 287. Sepp 1762-I860 VI p. I5, 145, I54, I6I, VII p. III, 2, I3, 24, 42, 84, I8I, 209, 2II, VIII p. 4, 13, 67, 132; G p. I64.

233 Eijndhoven, G. L. van. Haarlem. Lepidoptera, Acari. R.

234 Eldik, H. C. L. van. 's Gravenhage. Coleoptera. Everts I922 p. XII.

235 Elliger, Otmar. Hamburg I9. II. I666-St. Petersburg 9/20. XI. I735. Painter. Probably he is the author of a M. S. in A (Engel in Tijdschr. v. Entomologie LXXX, 1937, Verslag 70e Winterverg. p. XVI). In that case he collected insects. Thieme, Allg. Lexik. der bildenden Künstler, X I9I4 p. 467 (b) ; van Zuiden, in Feest-Bundel Dr A. Bredius 1915 I p. 292 (b); van Gool 1750-'I, I p. 243 (b). Molhuijsen I9II-'37, VI Kol. 476 (b).

236 Elout, Cornelis. I4. JII. I7I4 Haarlem-9. XI. I779. Cabinet described in Register van Verkoopingen van Schilderijen $n^{\circ}$ Io, sold Haarlem 5. IV. I780 (Archives, Haarlem); Lugt $1938 \mathrm{n}^{\circ}$. 31 15 .

237 Engelbronner, C. E. D'. Amsterdam. Merchant. Sander i783 I p. 587 (v 20. VIII. 1777). Cf. Grill. Catalogue 4. XII. I804 (B n $\left.{ }^{\circ} 746 \mathrm{a}\right)$.

238 Enschede, Jan (Johannes), J.zoon. Amsterdam 25. II. I706-Haarlem 2\%. VIII. 1775. Lived Haarlem, Kraijenhorstergracht. Merchant in linnen. Cabinet sold Haarlem I6. IV. 1776 (Lugt 1928 n $^{\circ}$ 2529, Ekama 1874 p. IO3).

239 Enschede. This village possesses a Museum of Nat. Hist. "Grensland" (Musea 1938 n $^{\circ} 74$ p. II6). R.

240 Entomological Society, Dutch. Once possessed a collection, which was given to Agricultural Highschool of Wageningen (van der Wulp I895; Horn \& Kahle 1935-'7 p. I9I).

24 I Erasmus, Desiderius (Gerrit Gerritszoon). Rotterdam 28. X. I467-Basel I2. VII. 1536. Lesser 1744 p. 47 and 1756 p. 59 says that he had a collection which came to Bonifacius Amersbach in Basel, from whose heir: 
it was bought by the town. In 1744 it was still there. Mollhuysen I9I I-'37 V p. I59.

242 Erdman, D. A., 's Gravenhage. Shells. R.

243 ERNST see Roeters (Roetert).

244 ERnST, J. F. Amsterdam I7... (Vosmaer 1790).

245 Esschers, L. N. Amsterdam I759 (Vosmaer 1790).

246 Eversdijck, H. C. J. van. "Secretaris van den Edelen Hove van Holland, Zeeland en West-Friesland". 's Gravenhage. Catalogue of his cabinet in A $n^{\circ}$ 104, sold in the Hague 28. IV. 1766 after his death (also Vosmaer I790, Lugt $1938 \mathrm{n}^{\circ}$ I525). Anonymus 1767 p. 328.

247 Everts, Jonkheer Dr Edouard Jacques Guillaume. 's Gravenhage I2. V. 1849-9. VI. 1932. Secondary Teacher in the Hague, well known for his work on Coleoptera. Collection in Museum Leiden and Museum Amsterdam. Horn \& Kahle I935-'37 p. 7o. For his life and work see Tijdschr. v. Entom. LXXVI, p. $1-46$.

248 FAGEL, Benjamin, 's Gravenhage bapt. I4. VIII. I642-I4. VI. I705. "Raadsheer in den Hove van Holland". Boehmer 1785-9 I p. 387 quotes the Sale Catalogue of his collections of shells and zoophytes:'s Gravenhage I709 (also Vosmaer I790). Valentijn I726 III p. 562, I754 p. 46, 56; Lesser 1756 p. 65 ; Kalff I928 p. 207 (m); Molhuijsen I9II-37 iII Kol. 382 (b).

$249 F_{A Y}$ DE LA = la Faille.

250 Faille,... DE LA, “Hoofdofficier der Stad Delft". Cabinet about I700. Valentijn I726 III p. 56r, I754 p. 45; Rumphius I705 p. 67.

25 I FaIlle, Cornelis De la. "Oud-Schepen te Delft". Valentijn i726 III p. 561, 572 (d), I754 p. 45, 56, 63, 66, 67; Murray I904 I p. I48 note 2 (refers to de la Combe de Vrigny, Travels through Denmark and some parts of Germany. London I707 p. 6); Uffenbach I782 III p. 335, 337, 340 (v I7IO); Seba Thesaurus III p. 138.

252 FAIlle, Johan Bernard DE la. 's Gravenhage. "Auditeur van de Kamer van Reekening". Valentijn I726 III p. 562, 570 (d), I754 p. 17, 19, 25, 46, 51, 52, 54 (d), 63, 64, 66, 67; Argenville 1742 p. 215 (de la Fay), I757 p. $147,384,1780$ p. 342 ; Seba Thesaurus III I758 p. I38; Gersaint 1736 p. I8; Lamy 1930 p. I3 note 4 ; Houttuijn 176r-'73, 16, p. 57 ; Boehmer I785 I p. 39I mentions his sale-catalogue: the Hague I73I (Gersaint says 1736 : sold for about 4 years, Lamy probably for this reason gives I732); Lesser I756 p. 65 ; Kalff 1928 p. 207 (m).

253 Faillie see Faille.

254 Fallie see Faille.

255 Favrod de Felle.ns, J., Rotterdam, Butterflies, Sepp 1762-i860 III p. I49.

256 Ferguson van der Meulen. Cabinet sold i76r in Amsterdam (Vosmaer I790). 
257 Feytema. Two brothers (Feijtemaas) Amsterdam. Rumphius I705 p. 67, 86, 99, I 19, I60, 289, 290 ; Valentijn I726 III p. 56I, I754 p. 45.

258 Feitema see Feytema.

259 Fierings (Fierentis, Fierens), Jacobus. Bookseller in Middelburg 1642I669. Nagtglas I888-'93 II p. 214 (b); Monconys I665 I p. 582 (v I663); Kanold 1727 p. 205.

260 Fierentis see Fierings.

26I Fierens see Fierings.

262 Fischer, F. C. J. Rotterdam. Lepidoptera, Trichoptera. Lempke 1936 p. 239. $R$.

263 Fokker, Mr A. J. F.; Zierikzee. Insects, Heteroptera, Homoptera. (Wulp I895 p. 17); Everts 1922 p. XII; Horn \& Kahle I935-'7 p. 79 (Pal. Hemipt. \& Coleopt. 1928 to Museum Leiden). R.

264 France van Berkhex, Johannes Le, Leiden 23. I. I729-I3. III. I8I2. Lector Nat. Hist. Leiden (I773- \pm 1796 ) (Francq I773). Van der Aa I 852 -'78. V (b) ; van der Klaauw 1926 p. 4, 52, 53, I25, I26; Maitland 1855 p. I68; Suringar 18672 p. 266; Francq I769-I8I I, a.o. III I p. 22 ; Björnståhl I782 V p. 430 (v I775); Ehrhart I783 p. 243 (v I782); Jaeck I826 p. 221. During his life he had to sell his books and collections. Catalogue of books and (p. 4I) natural history to be sold $20 . \mathrm{X}$. I783 seq. at Amsterdam ( $\mathbf{A} \mathbf{n}^{\circ} 2, \mathbf{B} \mathrm{n}^{\circ} 470$ ); Catalogue of his large collections of animal plates, of comparative anatomy, of minerals and shells to be sold at Amsterdam 29. III. 1785 ( $\mathbf{A} n^{\circ} 2, n^{\circ} 93$ A, Lugt $1938 n^{\circ} 3851$ ).

265 Franeker. This town once possessed a well known University, with the necessary anatomical cabinet. Uffenbach 1754 II p. 280-'7 (v i710, anatomy above senate-room); Dethmar I828-'4I p. I85-'8. Now a municipal museum contains some reminescences of the old glory (Musea 1938 p. $120 \mathrm{n}^{\circ} 78$ ).

266 Frank, Jean Guillaume. 's Gravenhage. Cabinet of birds, sale catalogue 21. IV. I762 p. 81 (B $n^{\circ}$ 291, Lugt $1938 n^{\circ}$ 1219).

267 Frank, G. A. Natural history agent. Blasius I880 p. 9, I4. British Museum I906 p. 354.

268 Fransen, C. died about r88o. Insects. Horn \& Kahle I935-7 p. 34I (coll. now in Museum Leiden).

269 Franssen, Dr. C. J. H. Butterflies now in Museum Maastricht. Lempke 1936 p. 239.

270 Fremery, Prof. Dr Nicolaas Cornelis DE. Overschie 1O. I. I770-Utrecht I5. XI. I844 Prof. med. Utrecht (I795-I844). Catalogue of his Museum (sold Utrecht, I. VII. I856) in A n ${ }^{\circ}$ I Io, p. I-26. Van der Aa 1852-'78 V; Maitland I855 p. I67; Murray I904 II p. 248; Witkamp I888 p. 306-8; Horn I831 I p. 377 (v 1830). Molhuijsen I9I I-'37 III Kol 419 (b).

27 I Frena. cf. Cramer I779-82 III p. I39.

272 FRINDS see Vriends. 
273 Gaillard see Kaillard.

274 Galen, H. G. van. Aalten. Butterflies. Lempke 1937 p. 245 . R.

275 Galitzine? Cabinet nat. hist. (Lugt $1938 n^{\circ} 3563$ ) sold Amsterdam 28. IV. 1783 .

276 Gaubius, Prof. Dr Hieronymus David. Heidelberg 24. II. I705-Leiden 29. XI. I780. Prof. Med. et Chem. Leiden (I734-I775). Van der Aa I852-'78 V (b). He possessed a cabinet of anatomy and natural history. Vosmaer 1790 mentions its sale without giving a date. Pallas $1766_{1}$, p. 209 , 262; Boddaert I768 p. XIX, 26I, 326; Houttuyn I76I-'73, I7 p. 350 (Gaubius possessed objects from Boerhaave's cabinet); Anonymus 1767 p. 325; Suringar 1866 p. 273; Bijdr. Meded. Hist. Gen. Utrecht 31 I9Io p. 4I8, 480, 33 I9I2 p. 400; See also UBA M. S. Cat .II n ${ }^{\circ}{ }_{1377}$; Sander I783 I p. 5 I9 (v I777); Ferrner I9I0 p. 478, 480 (v I759); Garampi I889 p. 200 (v I762); Beckmann I9I2 p. 400 (v I762); Desjobert I910 p. 9, Io v I778); Argenville I780 p. 347; Hublard I9ro p. 84. Molhuysen I9I I1937 III Kol. 43 I (b).

277 Gavel, Johannes. (Jean Gavelle), bapt. Middelburg. 26. VIII. I7I4-21. X. I757. Cabinet, insects, sold Middelburg, I. XI. I758. (B 256I, Vosmaer 1790); Molhuysen I9I I-'37 III Kol. 432 (b).

278 Geerts, S. J., 's Gravenhage. Shells. R.

279 Geevers see Gevers.

280 Geijskes, Dr D. C. Paramaribo, Surinam. Entomologist of Agricultural Experiment Station. Neuropteroidea. R.

281.Genege, Zara Aletta van, widow of Nicolaas Schaghen (in his life "Buitengemeen Raad van Indiën en Landvoogd van Amboina"): (Valentijn 1726 III p. 560, 1754 p. 44) (cabinet of shells later in the possession of W. Valckenier).

282 Genootschap see Middelburg, Rotterdam.

283 Gerdes, E. Teacher in Amsterdam, founder of the Dutch Entomological Society (Wulp I895). Horn \& Kahle I935-7 p. 88: Dutch Coleoptera to Mac Gillavry.

284 Gervais, D. surgeon in 's Gravenhage. Anatomical cabinet sold before 1790 (mentioned by Vosmaer I790 without year).

285 Geuns, Prof. Dr Stephanus Johannes van (Matthaeus zoon) Groningen 18. XI. 1767-Utrecht 16. V. I795 Prof. Med. Bot. et Phys. Utrecht 179I, et Chem .I794. Catalogue of his anatomical cabinet sold 13. VI. I796 in Utrecht (UBA). Molhuijsen I9I I-'37 I Kol. 932 (b).

286 Gevers, Abraham. Rotterdam 2. IX. I712-I5. X. I780 (Ned. Leeuw I92 I Kol. I35). "Consiliarius primusque urbis Roterodamensis consul, praefectus sylvarum Hollandiae et West-Frisiae, Soc. Ind. Or. Director”. Cabinet of birds, shell, zoophytes, insects etc. Argenville I780 p. 337 (Gevres); Rotterd. Courant 28 Aug. I774 (visit of Archduke Maximilian), 2I Mei 1778 (Duchess of Chartres and Princess de Lamballes); Cramer I779-'82 
I p. IIo, II7, II p. $5,27,39,72,73,85,87,99$ I00, IOI, I22, I37, III p. 59, 71 ; Stoll 1788, p. I22 ;Pallas 1767-'77 IV p. 15, VI p. 8, IX p. 22, X p. 32, I766 1 p. $183,188,1766_{2}$ p. 187 ; Boddaert I768 p. 228, 234, I770 VI p. 9; Anonymus I767 p. 325; Stresemann 1923 p. 120; Courtanvaux I768 p. I80 (v I767 d); Björnståhl I782 V p. 508 (v I775); Honoré I779 p. $27-28$ (v 1776 ); Sanders 1783 I p. 475 (v I777); Francq 1773 p. 36 (m) ; Molhuysen I9I I-'37, I Kol. 934 (b) (see also UBA M. S. Cat. II p. I75-6); Hublard I9Io p. 84; Museum was sold Rotterdam i787 (Hagen 1862 p. 37.7 ; Vosmaer I790; UBA; M. S. in Brit. Mus. Nat. Hist. Dept. Moll. and R. Soc. London).

287 Gevers Arnould. (shells Anonymus i767 p. 324).

288 Gevers son of Abraham (Courtanvaux I768 p. I80 v i767, “M. Geevers fils est Dir. de la Cie des Indes Occ.”).

289 Gevers, two brothers. (Thouin I84I p. I94 v I795).

290 Gevers Temminck I808-'9 p. 53 (menagerie de M. Gevers a Rotterdam), VII p. 66 (cabinet Geevers); G I938 p. I62.

29I Geverts see Gevers.

292 Gevres see Gevers,

293 Ghysels, Johannes. Clergyman in Goes. Cabinet sold 19. X. 1785 (B n ${ }^{\circ} 491$, Lugt $1938 n^{\circ} 3943$. Are the shells and naturalia from him or were they added to the sale?).

294 Gilhuijs, Henri J. Butterflies. Horn \& Kahle I935-7 p. 343 (gift 1902 to Leiden Museum).

295 Gils, van. Coleoptera (I922 to Mac Gillavry) Horn \& Kahle I935-'37 p. 287 296 Glockner probably Klöckner (Titius 1783 p. 193: Arzt Dr Glockner).

297 Goedaert, Joannes. Painter. Middelburg. I620-i668. Van Gool 1750-I, I, p. 4I (b); de la Rue I734 p. 33 (b); Nagtglas I888-'93 II p. 267 (b); Van der Aa I852-'78 V (b); Molhuijsen 19II-'37 I Kol. 944 (b); Monconys 1665 I p. 582 (v I663); Well known is his Metamorphosis 1662 in 3 vol. $1662,1667,1669$. (the last posthumously by his wife) in which he describes the insects he had reared.

298 Goel see Goll.

299 Goemans, Dr Hubrecht. Dreischor 14. III I803, physician in Zierikzee (1827-'87). His collection after his death to the Zeeuwsch Genootschap (Gedenkboek I9I9 p. I07). Nagtglas I888-93 II p. 269.

300 Goes, Museum voor Zuid- en Noord-Beveland. (Musea 1938 p. 120 $\left.\mathbf{n}^{\circ} 79\right) . \mathbf{R}$.

301 Goll J., Baron Gol.l van Frankenstein. Cabinet in Amsterdam (Argenville 1780 p. 356). Sander visited him 1776 on his estate at Velsen (Sander 1783 I p. 580: Goel ; and p. 566 cabinet at Amsterdam: Gould).

302 Gondlach. Cabinet sold i760 's Gravenhage (Vosmaer 1790).

303 Goske. 's Gravenhage. Sculptor, cleaner of shells! Valentijn 1726 III p. 562, I754 p. 46. 
304 Gould see Goll.

305 Graaf, W. D. V. DE. Druggist Enkhuizen. Sepp I762-I860 III p. 42, 49, 90, 93.

306 GraAf, G. M. \& Henri William DE. Brothers. Collection of birds to Zoological Museum Amsterdam. Insects (Butterflies) I908 to J. Th. Oudemans (Horn \& Kahle 1935-'7 p. 93). Sepp I762-I860 VI p. I71, I92, I96, VII p. 4, I34, I42, 2 I I, VIII p. 8, 98.

307 GraAg, Miss M. J. DE. 's Gravenhage. Shells. R.

308 Grave. Here was a menagerie of the Count of Gueldres. Witkamp 1872 p. $\mathrm{V}$ (lions in $\mathrm{r} 389$ ).

309 's Gravenhage. Witkamp i 872 p. IV mentions old menageries of the Counts of Henegouwen in the Hague (lions I35I, falcons, boars I344, '45), see also Loisel I912 I p. I56, II 29. Storks were kept by the town 1586 (Moll in Die Haghe 1937). The anatomical theatre was "annesso alla chiesa cattedrale" in 1669 (Hoogewerff I9I9 p. 309). Terwen I855 p. 27I tells us it was then in the Juffr. Idastraat. Hegenitius I630 p. I39 (v before I627) saw 2 casuaries and other animals in the stables of the Stadhouder in Rijswijk near the Hague. Murray 1904 I p. I47 quotes from Granger, Hist. of England III p. 402, London 1779 and Lister Journey to Paris. London I823 p. 82: "The States of Holland presented a collection to Charles II". Thunberg I792-'94 I p. I9 visits I770 the Hortus medicus. Two travellers mention sirens and satyrs in the museum (Arlincourt I842 I p. 300, $\checkmark$ I 837 , Clausade 1835 p. I73 v I834). In the Hague were the Cabinets of the Stadhouder (see Oranje, Vosmaer) and near it the menageries of the Prince (Honsholredijk, Loo). The zoological gardens (Koninklijk Zoölogisch-Botanisch Genootschap van Acclimatisatie te 's Gravenhage) was founded r862, Dir. Dr. L. H. Verwey later R. T. Maitland, Dr A. C. Oudemans, Dr J. W. van de Stadt, W. Graaf van Bijlandt. (Zool. Garten II p. 233, V I 864 p. 407, IX p. 342, XI p. 323, XII p. 210, 374, XIII p. 333 , 343, XV p. 464, XVIII p. 391, XXII p. I57, XXXI p. 266 , XLIX p. 376 ; Nederl. Tijdschr. v. d. Dierk. II I865 p. I03, 207 ; Witkamp I872 p. XXVII; Loisel I907 p. 97, 98, I I8, I9I2 III p. 221 ; Blasius I880 p. 24; Musea 1938 p. I44 $\mathrm{n}^{\circ} 97$ ). Zoological collections also in Mus v. h. Onderwijs (Musea I938 p. I $38 \mathrm{n}^{\circ}$ 9I).

3 IO GRIffet. Druggist (still living in 172486 years old) Rotterdam. Cabinet. Rumphius I705 p. I60; Valentijn I726 III p. 562, I754 p. 46.

3 I I Grill, Anthony. Merchant at Amsterdam, settled I722, still alive 1774 (75 years old) (Björnståhl i782 V p. 453; Bijdr. Meded. Hist. Gen. Utr. XXXI I9Io p. 329 note); Argenville 1780 p. 355 mentions especially his minerals; we have two catalogues of this cabinet (if it have not been two!): Catalogus van sijn Rarieteitenkabinet, Amsterdam 1728, $8^{\circ}$. (Boehmer 1785 I p. 390, Murray I904 II p. 267) and Museum Grilliani ... Amsterdam Io. IV. I776, sold after his death (Boehmer I785 I p. 390, 
Vosmaer I790, Lugt $\left.1938 \mathrm{n}^{\circ} 2525\right)$. There were many members of the family in Amsterdam (Uffenbach I754 III p. 660 visited I7Io an assayer Anton Grill who possessed 2 parrots; Patin 1676 p. 162 a lawyer Grill, who possessed a cabinet of pictures, mentioned also by Misson 1722 I p. 34).

312 Groen, Warnar. Amsterdam. Cabinet of shells. Valentijn i726 III p. 56I, 565 (d), 1754, p. 25, 45, 49, 51, 54, 57, 63, 64, 65, 66, 67, 68, 69.

313 Groenewegen, Salomon van. Amsterdam. Cabinet of shells sold 2 I. VIII. I758 (Lugt I938 $\mathrm{n}^{\circ}$ IOI7).

314 Groenewegen, Jacob Cornelis, Hortulanus at Hortus medicus (1836I883) had a collection of insects (Sepp I762-1860 VI p. 92).

3 I 5 Groll, H. W., about I8I9-I900. Insects. Horn \& Kahle I935-'37 p. 346 (Rhynchota to de Vos and Mac Gillavry, Coleoptera to Everts and Veth).

316 Groningen. In Academia Groningana 16I4-I9I4, Groningen 19I4 much information about the University and its Professors can be found. For the anatomy see also p. 518-522, for the natural history collections, begun I 816 by van Swinderen see p. 3I seq., 40, 50 seq., especially $52-54$, 516-7. Cf. Th. van Swinderen, Index rerum naturalium in Museo academico Groningano, 1822 (A) with sequels (a Society: Genootschap ter Bevordering van de Nat. Hist. in Groningen, was founded to sustain the Museum). Mac Gregor 1835 p. 252 note (v 1833 ); Terwen I855 p. 693 ; Blasius I 880 p. $7-8$ ( $v$ I879). The Museum burnt down 1906. The new collections were given on loan to the Leiden Museum in 1934. Horn \& Kahle I935-7 p. 97. See also: Hegenitius I630 p. I5 (v between I6I6 and I627); Melle \& Postel I89I p. 14 (v I683); Uffenbach I754 II p. 235, 238 ( $v$ I710); Haller 1883 p. 95 ( $v \pm 1723$ ). According to Musea 1938 p. $147-8, \mathrm{n}^{\circ}$ I0O, 102 zoological collections may be found in Biologisch Archaeologisch Instituut der Rijksuniversiteit and Groningsch Natuurhistorisch Museum.

317 Gronovius, Abraham, Dr Jur., I.eiden 24. VI. I695-'18. VIII. I775. Brother of following. Librarian of the Leiden University. His books sold 30. V. I785 (B $\left.\mathrm{n}^{\circ}{ }_{485}\right)$. Perhaps no cabinet, but he edited Aelianus, De Nat. Anim. ( 1744 A). Haller I883 p. 109 ( \pm I725). Van der Aa I752-'78 V (b). Molhuijsen I9I I-'37 I kol. 985 (b).

318 Gronovius, Johannes Fredericus, $\mathrm{Dr}$ med., Leiden Io. III. I690-I760, where he was often "raad, schepen, burgemeester". Friend of Linnaeus, brother of preceding and father of the following. Naturalist, especially botanist (Flora Virginica 1739); Pisces belgii in Acta Upsal. 1744-'50 p. 36; Index supp. Lapideae I750; etc. Van der Aa 1852-'78, V (b); His books were sold after his death on 16. III. 1763 Leiden, the catalogue (B $\mathrm{n}^{\circ} 298$ ) containing p. 72 only some naturalia, most of which probably came in hand of his son (following). Argenville 1780 p. 348 ; Ferrner 19 Io p. 463, 484 (v 1759), Molhuijsen I9II-'37 I Kol. 989 (b). 
319 Gronovius, Laurentius Theodorus, son of preceding. Leiden I730-'77 (8). $D_{1}$ jur. "Raadsheer, Schepen" in Leiden. "Député de la Chambre des Comptes de la prov. de Hollande à la Haye". Naturalist: Museum Ichthyologicum 1754-'6 (A) (To Gronovius also is due the invention of preparing flat skins of fishes in a dry state, and preserving them in the manner of a herbarium. This collection came via France in the British Museum, see Gray in Ann. Mag. Nat. Hist. (2) 13, 1854, p. 4I); Bibl. Regni Anim. atque Lapid. (I760) (A); Zoophylacium Gronovianum (I758, '74, '8I) (A), etc. Large cabinet of corals, fishes, vermes, insects, reptiles, mammals, shells, stones, plants etc. etc. Argenville I780 p. 346, 348; Anonymus I767 p. 325 ; Voet I766 I p. 81, 87, 88, 89, I02, I03, II p. 4, I2, 34, 42, 51, 87; Pallas 1766 I p. 40, 4I, 18I, 209, 299, 301, 307, 334, 427; Boddaert I768 p. XIX, 50, 5I, 225, 26I, 370, 373, 379, 4I2, 540; Ferrner 1910 p. 463, 484 (v I759); Garampi I889 p. 201-'2 (v r762); Thunberg 1792-'4 p. I7. (v I770); van der Aa I852-'78 V (b); Argenville I780 p .346. His books (B $n^{\circ} 438$ ) were sold 5 . X. I778 seq., Leiden, followed 7. X. I778 by the Museum, the Index of which (following the Catalogus librorum) was prepared by Meuschen, forming also part 8 of his Miscellanea Meuschenii (preface of Boddaert to the posthumous part 3 of Zoophylacium Gronovianum, I781; the Catalogus librorum 1778; Vosmaer 1790). Cf. van Alphen whose Catalogue 1789 p. 146 describes a Sirena lacertina from Gronov's cabinet.

320 Grovestein, Mrs., "Épouse du Grand Ecuyer de la Cour à la Haye". (Argenville I757 p. 147, I780 p. 342). Pallas I7661 p. 204; Boddaert I768 p. 255. (Perhaps Grovestins?).

32 I Grovestins, Mrs. According to Vosmaer I790 the cabinet of Mrs Grovestins was sold to the Empress of Russia. See also Vosmaer I800 p. 35 I $\mathrm{n}^{\circ} 286$ (see Grovestein).

322 Guicherit, D. J., Cabinet sold 28. IX-IO. X. I8I8 in 's Gravenhage (part 2) (Lugt $1938 \mathrm{n}^{\circ}$ 9442).

323 Guillor, Jacob. Amsterdam, "Droguiste sur le canal qui est derrière la Bourse ou le Rokin, possède un des plus beaux (cabinets) et des plus nombreux" (Gersaint I736 p. 40).

324 Gutsche, I., Cabinet of shells etc. Sold I749 's Gravenhage (Vosmaer I790).

325 H..., surgeon in 's Gravenhage. Catalogue of Sale 8. XI. 1784 (B $n^{\circ} 48 \mathrm{I}$ part 2 failing) contains much nat. history.

326 HaAG, T. P. C., 's Gravenhage. Cabinet of birds etc. sold I760 (Vosmaer I790).

327 HaAgen (Hagen, van Hagen are probably the same) Practicus Poliater or Doctor in Amsterdam (Pallas 1767-'77, VII p. 27, VIII. p. I9 note; Houttuyn I76I-'73, 8 p. 228).

328 HaAn, Dr Wilhem DE, Amsterdam. 7. II. I80I-Haarlem IV I855. Con- 
servator at the Leiden Museum. Versl. en Meded. Kon. Akad. Wetensch Amsterdam III p. 399 (b); Alg. Konst- en Letterbode 1855, 21 April, 2 Juni, I858 p. 193 (b) ; M. S. and drawings in A (cf. Jaarboekje Natura Artis Magistra I857 p. 98); portrait in Boschma, Verslag Rijksmuseum Nat. Hist. Leiden 1936-37; Horn \& Kahle 1935-'37 p. Ior (Orthoptera to Mus. Leiden); Sepp I762-1860 V p. I70, VI p. 2. Catalogue of his books (no naturalia) sold 23. XI. I855, Leiden in E; G p. 269.

329 HaAn, De, Amsterdam. Cabinet of Shells. Valentijn 1726 III p. 56I, I754 p. I3, 45, 63, 64; Uffenbach 1754 p. 686 (v I710, speaks of de Hahn, this will be the same); Kalff I928 p. 207.

330 HaAR, Dirk TER, Nijmegen 25. X. I860-Kollum 29. VI. 1905. Notary in Kollum. Butterflies. Tijdschr. v. Entomol. 49 (b, portrait); his Dutch butterflies in coll. J. Th. Oudemans (Horn \& Kahle 1935-'7 p. 276). Wrote a well-known book on Dutch butterflies.

33 HaArlem. This town can boast of a mermaid. (Veryard I7or p. 7, v I682). De Hollandsche Maatschappij der Wetenschappen (founded I752; Verhandelingen $1754 \rightarrow$; Vrolik, W., Versl. Meded. Kon. Akad. afd. Nat. $\mathrm{V}, \mathrm{I} 856$, p. 13) possessed a collection of natural history which had been founded on a collection given by burgomaster Hasselaer of Amsterdam, who got it from Governor Mossel from the East Indies, and on the cabinet, of its Director D. van Lennep ( 1776 , see Muntendam 1938 p. 470). It was often visited when under the care of Van Marum (1777-1837), later on under Van Breda, it came, at least partly, to the Museum of the Zoological Gardens Amsterdam, where a M. S. Catalogue is present: Lijst der voorwerpen voor het Museum uit de verzamelingen van de Holl. Mij. te Haarlem verkregen: all sorts of lower animals. Argenville ı780 p. 357 ;Sander 1783 I p. 535 (v I777); Thunberg I792-'94 II 2 p. 257 (v I779); Björnståhl I782 p. 437 (v I775);. Honoré I779 p. I97 (v I776, if I may read Gesellschaft: Maskobey as Maatschappij); Eversman 1792 p. 79, 87, 89 (v I783); Forster I868 II p. 98, 99 (v I790); Rudolphi 1804 I p. I I9, I2I (v I802); Strick I8I8 p. I54 (v I817); Fliedner I83I p. 394 (v 1823); T'erwen I855 p. 72.

Teyler's Museum (Vrolik 1.c. p. 19) only collected physical instruments and fossils. Schaeffer 1794 p. I24 (v I787-'8); Forster I868 p. 98 (v I790); Anonymus I792 p. 77 (v I790); Sierstorpff I804 p. 555, 556 (v I802); Rudolphi I804 I p. I2I-23 (v I802); Niemeyer I823 p. 150 (v I806); Haupt I8I4 II p. 192-8 (v I8Io); Meiszner I8I9 p. 69, 71, 72 (v I8I ) ; Strick I8I8 p. I54 (v I8I7) ; Neill I823 p. I89 (v I8I 7); Fliedner I83 I p. 286, 394 (v I823-'4); Dethmar I838-4i p. I47 (v I835-'7); Trewen I855 p. 7I; Blasius I880 p. I6 (v I879); Murray I904 II p. 272; Musea 1938 p. $157 \mathrm{n}^{\circ}$ I Io.

In the Koloniaal Museum in the house of Hope, the Palace near the "Hout" (Blasius 1880 p. I7, v 1879) animal products were shown, this museum is now in the Koloniaal Instituut Amsterdam. 
332 HAAS, F. DE, Rotterdam. "Conseiller de nos Indes Orientales". Cabinet of shells sold 23. III. I746 (Vosmaer I790; Lugt I938 $\mathrm{n}^{\circ}$ 631). Lyonet bought the best shells from his (de Hase) collections (Hublard I9Io p. 87).

333 Hacquet see Acquet.

334 Hagen, van see Haagen.

335 Hagoort. Cabinet of diverse naturalia sold 1774 Amsterdam (Vosmaer 1790).

336 HaGLE, THE see 's Gravenhage.

337 HaHn, DE see de Haan.

338 Hahn, Prof. Dr Johann David. Heidelberg 9. VII. I729-Leiden I784. Prof. phil. nat. Utrecht (I753-'55); Prof. med. Leiden (1755-'84). "S'est formé surtout en zoophytes et autres animaux-plantes une collection nombreuse et choisie" (Argenville I780 p. 337; Bijdr. Meded. Hist. Gen. Utrecht 31, I9IO p. 404, 406, 414-27, 433); Beckmann 1912 p. 418 (v 1762); Björnståhl I782 p. 489, 490 (v 1775); Ehrhart I783 p. 234, 245 (v I783). His collection was sold Leiden Io. I. 1785 (Vosmaer 1790; Cat. in B see p. I79, 193), it was bought by the Leiden University (Siegenbeek I829 II p. 92). Van der Aa I852-'78. VI (b).

339 Hahn, Frederik Hendrik. Obviously a son of preceding. Insects (Cramer I779-'82 I p. 7I).

340 Hallegraeff. Zutphen. Insects. Sepp i762-I860 VII p. III.

34I Hanau, Ernst Frederik. Cabinet sold 24. XII. I822-3. I. I823, Amsterdam (Lugt $1938 \mathrm{n}^{\circ}$ 10363).

342 HARDerwiJK. This town is well known for the many celebreties that took their doctor-degree at its University. I only know one traveller mentioning its anatomy: Anonymus 1787 in Bernouilli's Sammlung VII p. I66 (kind communication Prof. Arndt Berlin). See also Bouman, H., I844-'47. Geschiedenis van de voormalige Geldersche Hoogeschool. Utrecht 2 dln. (UBA).

343 Harmsen, G، I. A., Haarlem. Shells. R.

344 HaRst, J. C. VAN DER, J. J.zoon, conservator of the shell collection of the Zeeuwsch Genootschap, I895-I9I8 (Gedenkboek I9I9 p. IO9).

345 Hartoog see Kinschot.

346 Hase, DE see de Haas.

347 Hasselt, W. van, Bloemendaal. Shells. R.

348 Hasselt, Dr Alexander Willem Machiel van, I8I4-I6. IX. 1902. Inspector of medical service East Indies. Specialised on spiders. Wulp I895 p. 20, 46, 48; Horn \& Kahle I935-'7 p. 287 (Spiders to Mus. Leiden); Nederl. Entomol. Vereen., Verslag 6. VI. 1903 p. 32-34 (b); Tijdschrift v. Geneesk. I902 (b) ; G p. I67.

349 Havart, Peter, Merchant in Derdrecht and Rotterdam. Cabinet of birds (Argenville I780 p. 338). Nozeman I770 I, however, figures birds from the cabinet of Peter Havart in Rotterdam. 
350 Havelaar, Lodewijk Jacobus. Sepp 1762-I860 VII p. 14I; Horn \& Kahle I935-'37 p. I06 (Lepidopt. via Verhuell to Mus. Leiden) p. 349; G p. I67.

35 I Haverhorst, Breda. Lepidoptera and Hymenoptera Aculeata. Lempke 1936 p. 239, also 1937 (Ts. v. Ent. 80) p. 245 (now in Mus. Rotterdam). R.

352 Haverschmidt. Birds. R. Collection bought by Zool. Mus. Amsterdam (Nat. Artis Magistra).

353 Heenck, Engelbert. Custos Cabinet Natural History Leiden (van der Klaauw 1926 , p. 126; Suringar 18672 p. 8-9).

354 Heent see Hent.

355 Heere, J. de, Governor of Amboina. Cabinet of Nat. Hist., Lepidoptera. Sold Amsterdam 18. IX. I77I (Lugt $1938 \mathrm{n}^{\circ}$ I960).

356 Heerholtz, C. W. His collection of birds was shown on the Amsterdam fair of 1834 .

357 Heerlen (Limburg). Geological Museum contains palaeontology (Musea 1938 p. $163 \mathrm{n}^{\circ}$ II6).

358 Heeteren, van. 's Gravenhage. "Directeur de la Cie des Indes Occid. de Rotterdam" (Argenville r780 p. 347).

359 Heeze, I. DE. Cabinet of shells sold Amsterdam I77I (Vosmaer I790).

360 Heidan, Carl, Leiden, Secretary of accounts. Thesaurus antiq., conchyl., corall. Lugd. Rat. I69o (Murray IgO4 II p. 28I) or I697 (Lugt I938 $\mathrm{n}^{\circ}$ 165).

361 Heyst, van. Collections from Surinam, sold to Zool. Mus. Amsterdam (Natura Artis Magistra).

362 Heinsius, Nicolaas (Daniëls zoon). 20 Juli i620 Leiden-7. X. I68I 's Gravenhage. Author and poet. Had a cabinet in Leiden (Kanold 1727 p. 59) if indeed Kanold meant this celebrated person. (Van der Aa 1852-' $78 \mathrm{VI}$ b); Molhuysen 191 I-'37 II Kol. 557 (b).

363 Helder, Den. Here the Zoological Station of the Nederlandsche Dierkundige Vereeniging was founded 1888 . It contains collections from the adjoining seas (Musea 1938 p. $165 \mathrm{n}^{\circ}$ II 7 ). A "Natuurhistorisch Museum" contains more general topics (Musea I938 p. I64, $n^{\circ}{ }_{116 a)}$

364 Hellebrekers. Rotterdam. Bird's eggs. R.

365 Helmolt. "Geheimschrijver van de Raad van Justitie tot Batavia". Cabinet of shells sold in Amsterdam (Valentijn i726 III p. 560, 564, I754 p. I4, $44,48,63,64,65,67)$.

366 Hem, Mr van Der. Lawyer. Amsterdam. Cabinet (Patin I676 p. I62 v i67 I, copied by Misson I722 p. 34).

367 Hemstra, Cornely van. Menagerie in St. Gerlach near Maastricht, later castle Groeneveld near Utrecht, later Touraine (Loisel I9I2 III p. 87).

368 Henrard, Dr J. Th., Oegstgeest (with his son J. B. Henrard). Conservator Herbarium Leiden. Shells. R.

369 Hens, P. A., Valkenburg, burgomaster. Birds. R.

370 Hent, Rentre. Amsterdam Cabinet (Kanold I727 p. 182) Probably Roeter, Ernst? See also Heenck? 
37 I Herman, Prof. Paulus. Halle 30. VI. 1646-Leiden 25. I. 1695. Prof. bot. Leiden. Collected in India, Africa, Ceylon ( 1672 seq.). He possessed a large herbarium himself and also a cabinet of animals in liquor (Melle \& Postel I89I p. 21, v I683) in his home next to the Botanical Gardens. Stresemann 1923 p. II5; Van der Klaauw $1926_{1}$ p. I, 46 note 25 ; Siegenbeek I829 II p. 74, 78, I52, I53, Toev. en Bijl. (b) ; Van der Aa I852-'78 VI (b). In literature I found a sale catalogue 29. VI. I7 I Leiden: Musei Indica catalogus, continens varia exotica animalia, insecta... quae collegit P. Hermann (Hagen 1862 p. 360, Dryander I798-1800 I p. 224, Boehmer I785 I p. 387).

372 's Hertogenbosch Nat. Hist. in Centraal Noord-Brabantsch Museum van Prov. Gen. v. K. \& W. in N. Brabant. (Musea 1938 p. $167 \mathrm{n}^{\circ} \mathrm{r} 20$; founded 1836. Vrolik Verslagen Meded. K. Akad. Wet. Amsterdam, Afd. Nat. V p. I7). Blasius 1880 p. 27-28. Murray I904 (Catalogus van de rariteyten die op de rariteyt- en konstkamer van de stad 's-Hertogenbosch... I2mo I700).

373 Heukelom, Frans van. Amsterdam 18I I-'72 "President Kamer van Koophandel". Cabinet of shells and books to Zoological Gardens Amsterdam of which he was a president during many years. Catalogue in $\mathbf{A}$ $n^{\circ} 3254$ a (1864).

374 Heuvel, Martin van Den, Amsterdam. Manufacturer of gilt leather. Menagerie (Fuchs I9I6 p. 7, 55, the editor supposed this to be Isaac Heuvel, but Jensen I9I9 p. 45 , note 2 corrected this).

Heuvel, Pieter van den, Amsterdam. Cabinet of shells (Valentijn i726 III p. $56 \mathrm{r}, \mathrm{I} 754$ p. 45 ).

376 Heuvel, Pieter van Den. Middelburg. Cabinet of shells (Valentijn i726 p. 563, I754 p. 47) Nagtglas I888-'93 II p. 376-7 (m).

377 Hijmans, H. M., born about 187o. Coleoptera (to Mac Gillavry \pm 1905) (Horn \& Kahle i935-'37).

378 Hilversum. Museum voor het Gooi en omstreken. (Musea i938, p. i70 $\left.n^{\circ} 123\right)$.

$379 \operatorname{Hocy}(\mathrm{s})$, Dr van. 's Gravenhage. Cabinet of nat. hist. (Björnståhl I782 V p. 372 , v 1774 ). This is probably van Hoeij.

380 Hoeis, Dr Cornelis van, 's Gravenhage (physician and "membre du conseil", "polyater et senator"). 2. II. I7I7 - buried 22. VIII. I803. Cabinet. Pallas 1767-'77 VIII p. 41, IX p. 9, 1766, p. 168, 203, 1766, p. 177, I79; Boddaert I768 p. XIX, 209, 210, 250, 465, I770 III p. 25 ; Houttuijn. I76II773, 17 p. 356; Voet I766 I p. 4, 5, 9 10, 25, 56, 85, 91, 92, 98, IOI, II p. 28,69; Anonymus I767 p. 328 (Vanhoué); Beckmann I9I2 p. 348 (v I762); Sander I783 I p. 60I (v I777); Titius I783 p. 222 (v I777); Björnståhl I782 V p. 372 (v I774 Dr van Hocy); Argenville 1780 p. 345; Molhuysen 191 I-'37 IX Kol. 381 (b).

38 I Hoeven, Prof. Dr Jan van der, 9. II. I80I-Leiden Io. III. i868. Prof. Zool. 
Leiden 1835-1868). Cabinet of skulls (Catalogus Craniorum... Lugd. Bat. I860, in UBA and A French ed. 1875). The sale catalogue of his library (E) mentions I zoophyte and 17 birds (p. 212). Van der Klaauw 1926 1 p. IO, 12, 21, 70, 75, 95, 1926 2 p. 2 seq.; Jaarb. K. Akad. Wet. Amsterdam I868 (b). Czermak I879 I p. I7 I, I73 (v I850); G p. 268.

382 Hoffmann, "Chirurgien major et pensionné de la Ville". Maastricht. Cabinet of fossils. Sander I783 I p. 609 (v I777) ; Argenville I780 p. 336; Vosmaer 1790 mentions his Sale Catalogue: Maastricht I784.

383 Hogendorp, Dirk, Graaf van, † 1759 Rotterdam "Raad der stad, rentmeester publieke kerken", "drossaard van Steenbergen", later "Ontvanger Generaal der Unie". Menagerie on his estate "De tuin Sion", N. W. of Delft, Corner Kerstange and Zwet. (Loisel I9I2 II p. 50; Biema i910 p. 85, v 1736). Van der Aa 1752-'78 VI (b).

384 Hollander, Pieter DE. 7. VIII. I7OI, Haarlem, buried 26. IV. I770 (Ekama I874 p. I03). Sale catalogue I4. VIII. I770, Haarlem contains nat. hist. shells (Lugt $1938 \mathrm{n}^{\circ}$. I86I).

385 Hollandsche MaAtschappij see Haarlem and van Marum.

386 Holleman, Wouter. Coleoptera (now Mac Gillavry) Horn \& Kahle I935-'37 p. I16.

387. Holthuizen, L. F., Amsterdam. Cabinet of insects (and birds?). Stoll I788 1 p. I29, I30, I33, I35, I44, I45, I54, I788 2 p. 67, 77, 79, 81, 85, 86, 93, I02, 104, I813 A p. 4, 7, 8, 10, 22, 23, 24, 28, 29, 32, 40, 43, 45, 46, 48, 50, 61, 62, 66, В p. 8, 9, I1, I5, C p. 7, 8, 12, I6, I7; Cramer I779-'82 IV p. II6, 207, 209, 214, 233, 235, Aanhangsel p. 22, 43, 54, 6I, 121, 125, 128, I32, I35, I37, I38, I4I, I44, I47, I50, 161, I62, I64, I67, I75 ; [Temminck I 808 -'9 VIII p. 82 : II (Holthuysen)].

388 Holthuysen. same as preceding? Cabinet of birds. Temminck i8o8-'9 VIII p. 82 : II.

389 Номоет. Cabinet of shells. Sold 1757 in Amsterdam (Vosmaer I790). Mentioned p. IX Catal. Cabinet Leers 1767 (see there).

390 Honert, J. van Den, $\dagger$ 1918. Dutch Macrolepidopt. to J. Th. Oudemans (Horn \& Kahle 1935-'37 p. 286).

391 Honselaersdy (Honsholredijk) near Delft. Here the Stadhouders (Frederik Hendrik) had a menagerie. Brom I9I5 p. I03 (v 1677); Morren, Th. Het huis Honselaarsdijk. Uitg. "Die Haghe". Leiden p. 58 [UBA] ; Tulp, Obs. Med., I64I, III Cap. LVI p. 274; Upmarck, Ein Besuch in Holland. (N. Tessin I687) in: Oud Holland I900 p. I46; Regnard I874 p. 37 (v I68I); Melle \& Postel I89ı p. 24 (v I863); Mountague I696 p. 53 (v I695); Motraye I727 II p. I84 (v I7I4); Shaw I709 p. 24 (v I700); Witkamp I872 p. VIII; Loisel I9I2 II p. 29 seq.

392 Hoogeveen, Catharina van. Wife to Robbert Padbrugge ("in syn leven buitengewoon Raad van Indiën en Landvoogd van Amboina”). Shells. Valentijn I726 III p. 560, I754 p. 44. (Commenced to collect about I683). 
393 Hoogeveen. Douairière. Collection of shells given 1883 to Leiden Museum (Horst \& Schepman 1908 Introd. p. VIII) ; G p. 168.

394 Hoop, D. van DER. I864-I925. European Coleoptera 1927 to Mus. Amsterdam (Horn \& Kahle I935-'37 p. 286). Everts 1922 p. XII.

395 Hoos, Johannes. Collection of shells sold Amsterdam 19. XII. I763 (Lugt $1938 \mathrm{n}^{\circ} \mathrm{1336)}$.

396 Horne, Prof. Dr Johannes van. Amsterdam i62 I-Leiden 5. I. I670. Prof. anat, et med. Leiden (I65I-I67I). Van der Aa I852-'78 VI (b); his signature in Album Amicorum Blasius UBA; Lucae I854 p. 72, I 24 ( $v$ about 1665 ). His museum is mentioned by Valentinus I7I4 II p. I9 and Kanold I727 p. 20I; Molhuijsen 19II-'37 VII Kol. 624 (b).

397 Houbakker, Jean. Amsterdam. Insects. Uffenbach i754 p. 583, 584, (v i7io). 398 Houḱ, van, see Hoeij.

399 Houtruyn, Martin. I720-about I786. Dr med. Hoorn, Amsterdam, Rozengracht. Well known for his work on the three kingdoms of Nature following Linne's new System (Houttuijn I76I--73). He possessed a large cabinet. Björnståh1 I782 V p. 467, 469 (v 1774); Sander I783 I p. 578 (v I777); Titius I783 p. 195 (v I777); Ehrhart I783 p. 262 (v I782); Smith 1796 I p. 22, 23 (v 1786); Knorr I770-'75 IV pl. XXVIII, XXIX, XXX; V pl. III, IV, V, VIII-XXV, XXVII-XXX; VI pl. I-XXX; Cramer I779-'82 I p. 60, 76, I22; II p. 7, I5, 50, 64, 65, IO3; III p. III; IV p. 197, 234, 236; Stoll I788 1 p. 66, 67, 69, 70, 7I ; I7882 p. 5I, 53, 54; I8I3 A p. 24; Francq I769-I8I I III p. I8I ; I773 p. 35 ; Argenville I780 p. 347, 35I-54; Vosmaer I790 (cabinet sold I788 and I789); Murray I904 II p. 292 gives two catalogues: Musaei Houttuiniani, I regn. anim. Amsterdam i786 (also Engelmann i86i II p. I607) and: Animalium Musaei Houttuynianae rudera Amsterdam 1787; Van der Aa 1852-'78 VI (b) ; Maitland I 855 p. I68.

400 Hove, M. TEN, 's Gravenhage, cabinet sold $175^{2}$ (Vosmaer 1790).

40 r Hovius, Jacob. 1724-180I. Physician Amsterdam. Collection of pathological bones to Collegium Chirurgicum Amsterdam 1773. Nuyens 1928 p. 48. Gedenkboek 1932 p. 185.

402 Huydecoper van MaArsseveen, Jonkheer Johan, had a menagerie on his estate Groeneveld near Eemnes I797-I835. (Terwen I855 p. 144).

403 Huygens, Theodorus "Raadt en Oud-Schepen” Amsterdam. Cabinet. Seba I734-'65 I p. 73; Lugt I938 n 539 (sold Amsterdam 9-IO. V. I74I.

404 Hurgronje see Snouck Hurgronje.

405 IJ see also $\mathrm{Y}$.

406 IJver, P. Cabinet (Vosmaer I790) sold Amsterdam i788.

407 IsAAC, Adriaan, mentioned in Gedenkboek I9I9 p. IO4 is probably a mistake for A. I. Snouck Hurgronje. (cf. Nagtglas I888-'93 II p. 434). 
408 JANSE see Volkert.

409 Janse, J. A., Amsterdam. Butterflies. Lempke 1936 p. 239. R.

4lo Jansen, Abraham Jacob, born 1912. Dentist. Amsterdam Mollusks. R.

4I I Jansz see Volkert Jansz.

412 Joснемs, 's Gravenhage. Acclimatised lama's. (Loisel I9I2 III p. 87, I907 p. II5).

413 Joncheere, W. De. Dordrecht. Butterflies. Lempke I936 p. 239. R.

414 JonG, DE, "Boekhouder O. Ind. Cie". Cabinet of shells. Uffenbach 1754 III p. 656 (v I710). Is this the Johan de Jong mentioned: Rumphius I705 p. $19,36,42,86,99$, II 2 ; Valentijn I726 III p. 56 I ; I754 p. 54 ?

415 Juliaans (Juliaan, Julianus), L. Druggist Utrecht. Well known Cabinet of shells, insects etc. Argenville I780 p. 337 ; Voet I 806 I p. 48 II p. II, 12, 44, 50, 68; Cramer 1779-'82 I p. 98, 127, I30, II p. I I, I8, 94, I25, III p. 42, IV p. I65 ; van Phelsum I774 p. 53 ; Boddaert I768 p. XXIII ; Houttuijn I76I-'73 I2 p. 92, I7 p. 333; Sander I783 I p. 599 (v I777).

4I6 JurriaAnse, Jan Hendrik, born i866. Lepidopt. exot. 1932 to Mus. Leiden (Horn \& Kahle I935-'37 p. I28).

4'7 KaAs, P., Loosduinen. Shells. R.

418 KaAten, ten, see Kate.

419 Kabos, Dr W. J. Diptera. R.

$420 \mathrm{KAHN}$, Dr Amsterdam. Anatomical Museum. (Catalogue in UBA).

42I Kaillard, Jan (probably Gaillard). Merchant. Amsterdam. Zoophytes. Uffenbach I754 III p. 548 (v I7IO).

422 KalF see Calf.

423 Kamerling, Delft. Cabinet of shells. Valentijn i726 III p. 56I, i754 p. 45.

424 Kampen. Even in this town there was a lion-tower where 1477,1483 lions, sent via Spain and Portugal, were kept and propagated, so that 5 à 6 were given to the town of Lübeck (Witkamp I872 p. V, I888 p. 268-7i ).

425 Kappelhoff, Dr. med. Amsterdam. Insects. Cramer I779-82 II p. I39.

$426 \mathrm{KAs}$, Rotterdam. Bought insects from coll. Eyndhoven (see there).

427 Kat van Barendrecht at Dordrecht. Collection of birds bought at auction Klinkenberg I84I, which he had drawn by Schouwman (Van der Aa I852-'78 s.v. Schouwman, Aert).

428 Kate, Lambert ten, Hermanszoon. Amsterdam. Corndealer. Cabinet (Uffenbach I754 III p. 65I, 654, v I7I0), sold Amsterdam I6. VI. I732 (Lugt $1938 \mathrm{n}^{\circ} 4 \mathrm{I} 6$ ).

429 Kate, Hendrik ten, nephew of preceding. Merchant Amsterdam. Cabinet Uffenbach 1754 III p. 677 v I710, he had bought shells of Schijnvoet); Pekarskij I862 p. 543-'58 (v I72I).

430 Kate, C. G. B. ten. Kampen. Birds. R.

43 I Kate, J. ten. Struyck 1718 (in title).

432 Kate, ten...... Shells. (Valentijn I726 III p. 56I, I754 p. 45). 
433 Kate, R. ten. Zierikzee. Shells. R.

434 Kattenburg see Cattenburg.

435 KeIZERSKRoON. In the tavern of this name a beautiful collection of birds. each in its environment was shown (Garampi I899 p. 226, v I764).

436 Keijzer, Dr IJsbrand. Zevenhuizen I824-Apeldoorn I896. Physician in Middelburg (Noordstr.) Compiled Catalogues of the shell collection of the Zeeuwsch Genootschap, I869 and I879. Gedenkboek I9I9 p. I09; Nagtglas I888-'93 II p. 537 (b) ; De Man 1902, De geneesk. school te Middelburg. I825-'66 p. 66 (b). His collection came to his son.

437 KeIJzer, Ir P., IJzoon, I854---I937, son of preceding. Secondary teacher Apeldoorn. He sold the collection of shells, begun by his father, in 1936, principally to Museum Enschede.

$43^{8}$ Kerckring, (Theodorus? Med. Dr., since 1678 Hamburg, where he died I693, 2. XI.) Utrecht. Cabinet of anatomy (Patin I676 p. I55 v I67I).

439 Kerkhoven, Augustus E., † 1924. Apeldoorn. Coleoptera to Mus. Leiden (Horn \& Kahle I935-'37 p. I33, 360); Everts I922 p. XII.

440 Kien van Citters, Willem Aarnout. 28. I. I74I-22. IX. I822. "Raadpensionaris" Middelburg, "Bewindhebber O. Ind. Cie ter kamer Amsterdam". Lived on his estate Brakenburg near Arnemuiden. Nagtglas I888-'93 I p. 126 (b). Cabinet sold Middelburg 21. VIII. I798 (Lugt I938 n $^{\circ} 5800$ ).

$44^{I}$ Kienjet, D. J., Leiden. Shells. $R$.

442 Kigelaer see Kiggelaar.

443 KiggelaAr, François. † I723. Amsterdam "Commies ter secretarije van den Heer van Beaumont”. Seba 1734-'65 I (Introd. of Boerhaave); Valentijn I726 III p. 562, 570 (d) ; I754 p. II , 25, 26, 28, 46, 5I, 52, 54, 56, 63, 64, 65, 66, 67, 69; Argenville I780 p. 342 (Kigelaër); 1742 p. 215 (Kisglaër à la Haye); Lesser I756 p. 69 (sale catal. I723). This sale catalogue is also mentioned by. Murray Ig04 II p. 308.

444 Kimpe, W., Middelburg. Shells. R.

445 Kinschot, Cornelia van, widow of Willem Hartoog. Cabinet sold II. VIII. I788 Amsterdam. (Lugt $1938 \mathrm{n}^{\circ} 434 \mathrm{I}$ ).

$446 \mathrm{~K}$ IP, see Taelman.

447 KlaAssen, H. J. Amsterdam. Insects. Everts I922 p. XII.

448 Klijnstra, B. H., 's Gravenhage. Coleoptera. Everts 1922 p. XII. His son F. B. collects the same insects. $R$.

449 Klinkenberg, G. J. van. Utrecht. Amateur but he dealt in naturalia also. His cabinet bought by Lith de Jeude (L. d. J. 1856 pref.). In A is: Extr. du Catal. détaillé du Músée d'Hist. Nat. de G. J. Klinkenberg à Utrecht 1839 , also a Dutch edition. Birds to Kat van Barendrecht.

450 KJ.öckner (Clockner, Glockner, Glöckner). Dr Jacobus Christophorus. Physician Amsterdam. Cabinet. Nozeman I770 I; Cramer I779-82 I p. 82, IV p. 98, IOI, I I2; Björnståhl I782 V p. 460, 465 (v 1775); Sander r 783 I p. $488,535,577,578$ (v I777); Thunberg I792-'4 $\mathrm{II}_{2}$ p. 257 (v 
1779). Vosmaer 1790 mentions the sale of his cabinet Amsterdam 1783: divers naturalia.

45 I Kloкman, G. J., Laag Keppel. Insects. (Lepid.) Lempke 1936 p. 239. R.

452 Knogh. Dordrecht. Cabinet (Voet I806 II p. 28).

453 Knolter(s), Leiden. Cabinet (Valentinus I7I4 II p. 19; Kanold I727 p. 201 ).

454 Koening, Cabinet of shells sold Amsterdam i6. V. I770, catologue prepared by Meuschen and collected in his Miscellanea. Schröter, J. S. Einleitung in die Conchylienkenntnis nach Linné. 1783. p. 71.

455 KoK Blomhof see Cock Blomhof.

456 Kogelaer. 's Gravenhage. Cabinet Uffenbach I754 III p. 393 (v r7IO).

457 KoK, Jac. Amsterdam. Cabinet, especially shells sold 6 VII I768 (Lugt - $1938 \mathrm{n}^{\circ} \mathrm{I697)}$.

458 Kommer, Jan De. Collection sold i5. IV. I767 (Lugt I938 n I607).

459 Koning, P. P. De. Leiden (Mus. Nat. Hist.) Shells. R.

460 Koning, Willem De. Dordrecht. Cabinet of shells. Valentijn 1726 III p. 563 , 577 (d), r754 p. 5, 13, 24, 26, 27, 47, 49, 61, 64, 65, 66, 69 (cf. Martinet, Katechismus der Natuur II 1785 p. 387 ).

46I Kooker, Druggist Haarlem. Cabinet of insects. Uffenbach I754 III p. 522 (v I TIO).

462 Koornneef. J., Rhenen, earlier Velp. Hymenoptera, Lepidoptera. Lempke I937 p. 245. Everts 1922 p. XII.

463 Kops. Haarlem. Cabinet of birds sold i 769 (Vosmaer I790).

464 Kortebrand. Rotterdam. Raritäten-Kammer. (Willebrandt i76I p. 96 V I757).

465 Koumans, F. P., Conservator Museum Leiden. Shells. R.

466 Kramer see Cramer.

467 Kretschmar, J. van "Capitein en Commandant Gardes Koning Willem III”. Sale catalogue of cabinet, Delft, I8. X. I694 in UBA (637 G 5). Rumphius I705 p. 2 I7 (Krijtsmar).

468 KRIJTSMaR see Kretschmar.

469 Kronenberg see Burgh, Cronenburgh, Croonenburg.

470 Kruseman, Dr Gideon, born Haarlemmerliede en Spaarwoude 3. IX. I904. Assistant Zool. Mus. Amsterdam. Collects Diptera. R.

47 I Kuiper, Jac. G. Joh., de Bilt. Shells. R.

472 KujJK van Mierop, Rotterdam. Cabinet of shells. Valentijn i726 III p. 562, I754, p. 46.

473 Kuyl, Apeldoorn. Shells R.

474 LAARs, Michiel. Cabinet especially shells sold Amsterdam 9. IV. I764 (Lugt $1938 \mathrm{n}^{\circ}$ 1373, Vosmaer 1790).

475 LaERS, Rotterdam. Cabinet (Argenville i 780 p. 338).

476 Labouchère, A. M. Some time "Consul-Generaal in Nantes". Insects. (Sepp. I762-I860, III p.IO7-8, IV p. I48, V p. 32, 44, 67, 69. 
477 Lacourt, A. W. Leiden. Shells. R.

478 Lafaljé see Faille.

479 Lafaille see Faille.

480 Lafaillie see Faille.

48i Lambergen, Prof. Dr Tiberius, Reidsum 1717-Groningen 8. VI. i763. Prof. med. Franeker (175I) Groningen (1754). The sale catalogue of his books (B $\mathrm{n}^{\circ} 3 \mathrm{II}$ ) contains an anatomical collection (p. I47), it was sold $\mathrm{I}_{5}$. V. I764 (Vosmaer I790 who also mentions a sale catalogue 1782 Groningen of his cabinet of nat. hist. Molhuysen I9II-'37 X Kol. 507 (b).

482 Lambert, Mr G. H., Rotterdam. Shells. R.

483 Landschot, Mr. Joan van, "Burgemeester van Leiden, Meesterknaap van Holland", and his son Mr Joan Alexander v: L. "Scheepen van Leiden bewindhebber $\mathrm{O}$. Ind. Cie" have been acclimatising many animals especially birds (Francq I769-I8I I III p. I79-I80).

484 Lassara, DE. "Colonel des Cent-Suisses de M. le Stadhouder" 's Gravenhage. His Cabinet was bought for the Prince of Oranje (Argenville I780 p. 339, 342). Hublard r9io p. 84 (m, La Sarraz).

485 Latiers, H. J. H., Kerkrade, later Steyl. Lepidoptera etc. Everts 1922 p. XII, Lempke 1936.

486 Leefmans, Dr. S. Heemstede, Insects (Lamellicornia). $\mathbf{R}$.

487 LeEnhofF DE L'EspierRe, van. Burgomaster of Doesburg (was dead in 1860). Insects. (Sepp I762-I860 VIII p. 135).

488 Leers, Mr. Arnoud, Heer van Ameyde en Herlaar, "Ancien échevin Rotterdam", "Directeur du Trafic au Levant" etc., born I719. Cabinet of shells sold 20. V. 1767 Amsterdam (A edited by Meuschen and in his Miscellanea, followed by a M. S. Index of prices; Vosmaer I790; Lugt 1938 $\mathrm{n}^{\circ}$ 1618; Schröter, Einl. Conch. Kenntnis 1783).

489 LeessberG, Mr A. F. A., 's Gravenhage. Coleoptera. Everts I922 p. XII.

490 Leeuwarden. Here is a Friesch Natuurhistorisch Museum (Musea 1938 p. I79 $\left.\mathrm{n}^{\circ} \mathrm{I} 35\right)$.

49 I Leeuwen, Jan van. Butterflies to J. Th. Oudemans about I896 (Horn \& Kahle 1935-'37 p. 287).

492 Leeuwen. T. van. Sale of a cabinet of anatomy Amsterdam I7... (Vosmaer I790).

493 LeIDEN. Of course wild animals were shown on the fair (Loisel I9I2 II p. 50). On the "Burght" Jacobaeus (1910 p. Io) saw in 1650 all sorts of animals (see also Ferrner I9Io p. 462, who mentions a menagerie in 1759). Living animals were also kept during a certain time in the Botanical Gardens (Jacobaeus I9Io p. I0, v 1650; Ogier I910 p. 266, v I636; Brereton 1844 p. 42, v I634; Neill I823 p. I6r, v I817; Anonymus 1819 p. $98, v 1819)$. The history of the zoological teaching has been written 1926 by Van der Klaauw. It seems unnecessary to repeat here all the catalogues of the two celebrated collections of Natural History, that in 
the Theatrum anatomicum and that in the gallery of the Botanical Gardens. I may refer to Van der Klaauw 1926 p. 39 note 3, p. 43 note 7; Murray 1904 I p. 29, I90, 208, 287, II p. 326; Dryander 1798-I800 i p. 222-3; Boehmer 1785 I p. 380 ; in UBA there are many editions. Adding to the literature of the subject I may give the travellers mentioning the collections, (cf. Van der Klaauw p. 40 note 4 , p. 44 note 8 ). The Museum in the galery of the Hortus Botanicus (often called Museum Indicum) is mentioned by Sincerus 1649 p. 237 (m r619); Hegenitius I630 p. I03 ( $v \pm 1627$ ) Brereton I844 p. 92 (v I634); Ogier I9ro p. 266 (v I636); Evelyn I879 p. 30 (v I64I); Joly I672 p. I31 (v I646); Jacobaeus r910 p. 10 (v 1650); Birken I668 p. 198 (m 166I) ; Ray I738 I p. 31 (v I663); Monconys I666 p. I5I (v I663); Lucae I854 p. 78 (m \pm 1665); Hoogewerff 1919 p. XXXII, 99, 102, 254 (v 1667-'68); Brown I682 p. 8 (v I668); Patin I676 p. 164 (m I67 I); Pacichelli I685 p. 650 (v I674); Brom I9I5 p. 148 (v I677, '8 $d$ ); Melle \& Postel I89I p. 22 (v I683); Tessin I9I4 p. 73 (v I687); Misson 1722 p. I8 (v I687); Mountague I696 p. 7I (v I695); Bowrey 1927 p. 48 (v I698); Shaw I709 p. 27 (m I700); Erndtel I7II p. 80 (m I707); Uffenbach I754 p. 404-8, 495 (v I7II); Mottraye I727 II p. I78 (v \pm I714); Wright I730.(v I722); Haller I883 p. 40-43, IIo-2 (v I726); Poole I743 II p. 74 (v I74I ; Bocage I764 p. 9I (v I750); Monsanto I752 p. 28, 30 (v I750); Hude I784 XIV p. 100 (v I755); Willebrandt 176I p. 92 (v \pm 1757); Fermer 1910 p. 466 (v I759); Garampi I889 p. 201 (v I762); Beckmann I9ı2 p. 368 seq. (v I 762); Garampi I899 p. 214 (v I764) ; Lynar I78I p. I26 (v I77I) ; Björnståhl I782 V p. 385 (v 1775); Sander I783 II p. 527 (v I777); Titius 1783 p. 201 (v I777); Ehrhart I783 p. 240-4I (v I782); Anonymus I790 p. 100 (v I784); Smith 1796 p. 9 (v I786); Schaeffer I794 p. I2I (v I788); Thunberg I792-'94 p. I6 (v I770); Grimm I775 p. 302, 303 (v I774); Honoré I 779 p. I 42 (v I775); Ireland I789 p. 80 (v I789); Anonymus I792 p. 82 (v I790); Sierstorpff I804 p. 548 (v 1802); Rudolphi I804 p. I25-26 (v I802); Haupt I8I4 p. 2I4 (v I8Io); Anonymus I8I8 p. 5r (v \pm I8I4); Neill I823 p. I6I (v I8I7); Strick I8I8 p. I96-9 (v I8I7); Carter I8r9 p. 240 (v I8I8); Anonymus I8I9 p. 97-8 (v I8I9). I may at last mention: Valentini I7I4 II app. IX p. 2I (d); Suringar 18672 p. 266; Schotel, De academie te Leiden p. 59; Pallas $1766_{2}$ p. 56; $1767-77$ VII p. 27, VIII p. 20, IX, p. I2; Terwen I855 p. I87; Kanold 1727 p. 59 (d). The catalogue of Vosmaer 1790 mentions a catalogue (of sale?) of the Hortus dated I 735 ; G p. 28 a. o.

The Anatomical Cabinet was mentioned by: Neumayer 1620 p. 290 v I614); Sincerus 1649 p. 237 ( $m \pm$ 16I0); Brereton I844 p. 4I (v I634) Joly 1672 p. I3I (v 1646); Ogier I910 p. 266 (v 1636); Hegenitius I630 p. I05-13 ( $v$ before I627); Evelyn I879 p. 30 (v I64I); Jacobaeus I9ıо p. Io (v 1650); Ray I738 1 p. 32 (v 1663 ); Payen I663 p. 
56 (v I660 d); Monconys I666 p. I5 I, I55, (v I663); Lucae I854 p. 78 (m \pm I665); Hoogewerff 1919 p. XXXII, LVII, 99, I02, 254 (v I667-'68); Brown i682 p. 8 (v I668); Patin I676 p. I64 (m I67I); Pacichelli 1685 p. $652-55$ (v I674 d); Brom I9I5 p. I48 (v I677-'78); Regnard I874 p. 38 (v.168I); Veryard I70I p. 8. (v I682); Melle \& Postel I89I p. 22 ( $v$ I583); Tessin 1914 p. 73 ( I687); Misson 1722 p. 18 (v 1687); Mountague 1696 p. $72-95$ (v 1695 d); Shaw I709 p. 27 (m I700); Erndtel I7I I p. 80 (v I706); Uffenbach I754 p. 437-38 (v I7II); Sartre 1896 p. 50 (v I7I9); Haller I883 p. II I (v I726); Mottraye I727 II p. I78 (v \pm I7I4); Poellnitz I7.34 (m I7.32); Seba 1734-'65 III p. I08; Bocage 1764 , p. 91 (v I750); Willebrandt I76I p. 92 (v \pm I757); Ferrner I9Io p. 466 (v I759); Garampi I889 p. 201 (v I762); Beckmann I9I2 p. 373 seq. (v I762); Heinecken I769 p. 61 (v I768);Coyer I775 p. 273-75 (v I769) ; Sander I783 I p. 527 (v I777); Aikin 1920 p. 220 (v 1784); Smith I796 p. IO, II (v I786); Schaeffer 1794 p. I2I (v 1787-88); Guislain I842 p. 9I (v I84I); Clausade I835 p. I97 (v I834); Grimm I775 p. 302305 (v I774); Feller I823 p. 233 (v I775); Honoré I779 p. I43 (v I776); Ehrhart I783 p. 230-34, 243 (v I782); Ireland I789 p. I34 (v I789); Anonymus I792 p. 8I, 82 (v I790 d); Sierstorpff I804 p. 548-49 (v I802); Rudolphi I804 p. 132 seq. (v 1802); Silliman I8I2 p. I63 (v I805); Haupt I8I4 II p. 2I3 (v I8Io); Anonymus I8I8 p. 5I (v I8I4); Neill I823 p. I63 (v I8I7); Carter I819 p. 239 (v I8I8); Otto I825 p. 457 (v I822); Duchesne I834 p. 268 ( $v$ I827); Ladenberg 1830 p. I69 (v I828); Horn I83I p. 35I (v I830); Mac Gregor I835 p. I68-69 (v I833); Dethmar I838-'41 p. I09 (v I835, '36); Czermak 1879 p. I73-75 (v I850); Blasius I880 p. 22 ( $v$ 1879). I may also mention the catalogue in Valentini I7I4 II App. IX p. 53; Terwen I855 p. I88; Van der Klaauw $1926_{2}$; Geijskes \& Van der Klaauw I934; Blasius I880 p. 22 (v I879); Schotel, De academie te Leiden in de $16 e, 17$ e, 18 e eeuw p. 6r.

On the Rijksmuseum of Natuurlijke Historie Miss Gijzen wrote a doctors thesis (published while this paper was in print); Six, Overzicht der diensten door het Rijks Museum van Natuurlijke Geschiedenis te Leiden aan de Dierkunde bewezen, Murray I904 II p. 330; Temminck \& Laugier I838; Terwen I855 p. I87; Meiszner I819 p. 8I ( $\mathrm{v}$ I8I7, mentions the collection Brugmans got in Paris in stead of the old collections of the Stadhouder, a donation of the King to the University); Carter I8I9 p. 24I (v I8I8); Anonymus I8I9 p. 97 ( $v \pm$ r8I9); Otto 1825 p. 458 (v I822); Fliedner I831 p. 278 (v I823-'24); Jaeck I826 p. 212 (v I824); Duchhesne I834 p. 268 (v I827); Ladenberg I830 p. I70 (v I828); Horn I831 p. 355 (v I830); Mac Gregor I835 p. I68 (v I833); Lax I838 p. I30, (v 1837); Dethmar I838-'4I p. I09 (v 1835-37); Guislain I842 p. 9 I +2 ( I84I); Czermak I879 p. I73 (v I850); Clausade I835 p. I95 $(v \pm 1834)$; Blasius I880 p. 22 ; Beets in Jaarb. v. Gesch. en Oudh. van 
Leiden en Rijnland 1932-'33 p. 7I; Musea I938 p. I89 n 143.

Natural History may also be found in Rijks Museum van Geologie en Mineralogie (palaeontology: Musea I938 p. $183 \mathrm{n}^{\circ}$ I39).

494 Lely, Jacob van Der. Delft. "Raad en burgemeester". Sale of his shellcabinet 5. IV. 1796, Delft (Lugt $1938 \mathrm{n}^{\circ}$ 5429).

495 Lely, W. Van Der. Cabinet sold Amsterdam 1773 (Vosmaer I790).

496 Lempke, B. J. Amsterdam. Lepidoptera (Lempke I936 p. 239. R).

497 Lennep, van. This large family:"(Nederlands Adelsboek I921 etc. etc.) had many members interested in natural history. It was not always possible to find out which member was meant by some short note in literature. Vosmaer 1790 mentions the sale of a cabinet David v. L. Amsterdam 177I (I77I died Mr. David van Lennep "Commissaris, raad, schepen, weesmeester”, Haarlem, 3. X. I72I-20. V. I77I). Argenville i780 p. 357 speaks of an important collection of birds of a Van Lennep in Haarlem and in 1776 the Hollandsche Maatschappij in Haarlem got a collection of birds and stuffed mammals from her deceased Director the above mentioned David van Lennep (Muntendam 1938 p. 470). Cramer I779-82 I p. 86, 94, I46, II 3, I I, I6, I8, 21, speaks of Jacob Cornelis Sylvius van Lennep, probably a son of David mentioned above who married a Miss Sylvius, though I could not trace him in the genealogy. Cramer says he was a brother of the following Cornelis (son of David) and after his death his cabinet of insects went to that brother.

498 Lennep, Mr Cornelis van. Haarlem 22. III. I75I-Amsterdam I. II. I8I3. "Commissaris der Stad Amsterdam" etc. (Nederl. Adelsboek I92I p. 75). He collected insects, got the collection of his brother (preceding) and also possessed a cabinet of shells and zoophytes. Van Lennep 1862 (b); 19io I p. 27 ; Cramer I779-'82 II p. 49, 55, 76, 81, 83, 88, 100, I16, I27, 132, 135, I38, 141, III p. 8, 23, 28, 29, 30, 37, 61, 78, 91, I40, 144, I45, I48, I69 IV p. 8, 70, 7I, 225. Appendix, dedication and p. 175; Sepp I762-1860 I p. 74, II I p 6, II 4 p. 76, 80, II 5 p. I2, III p. Ir8; Eijndhoven I86I (via Prof. D. J. van Lennep, son of Cornelis, the collection came in the possession of v. Eijndhoven, see Tijdschr. Ned. Ent. Vereen. p. LXXXII); Nemnich I809 p. 37r (v I808); Horn \& Kahle I935-'37 p. 287 (Lepidopt. ex parte via Felder to Tring); Molhuysen 191I-'37 I Kol. 1263 (b).

499 Leth, Hendrik DE. Cabinet sold Amsterdam 30. IX. I 766 (Lugt 1938 n $^{\circ}$ I 560 ).

500 Lidth de Jeude, Prof. Dr Theodoor Gerard van. Tiel 8. VII. I788-Utrecht 23. XII. I863. Prof..med. Harderwijk 18I 5, Utrecht I8I9 and Director Veterinary School. He had a large collection (Lidth de Jeude $185^{8}$ ) which for the greatest part was bought for the British Museum. In $A$ is a $M$ : $S$. Catalogue of his cabinet. He bought the collections Draak, van der Capellen, Klinkenberg, Muller. Jaeck I826 p. 246 (v I824); Dethmar I838I84I IV p. 60, 6I (v I835-'37); Van der Klaauw 1926 2 p.' 2. Molhuijsen I91 I-'37 I Kol. 1274 (b); G p. I68, 277. 
501 Lieftinck, M: A., born 1904. Conservator Zool. Mus. Buitenzorg. Odonata. R. Everts 1922 p. XII ; Horn \& Kahle I935-'37 p. I56.

502 Lier, Mr Jạn van. Assen. "Receveur Général de la ville”, auteur du "Verhand. Over de slangen en adders in Drenthe". (178I). Argenville I780 p. 358. Van der Aa 1852-'78 VIII; Vosmaer I790 (cabinet sold Amsterdam 1786).

503 Limburg, A. Cabinet of animals and anatomy sold Amsterdam i720 (Vosmaer 1790 ).

504 Lindemans, J., Ermelo. Hymenoptera. R.

505 Loches, DE, Mrs., granddaughter of van Segveldt whose collection came through her to $\mathrm{Mr}$ de Loches, lawyer in Alkmaar. Partly sold, rest given by $\mathrm{C}$. $\mathrm{H}$. de Loches-Rambonnet to Museum v. Onderw. 's Gravenhage.

506 Lodeesen, W. A., Sepp I762-1860, VII p. 76.

507 Lodeesen, J. W. † I894. Butterflies Sepp. I762-i860 VIII p. I35, Horn \& Kahle 1935 -'37 p. 158 (Lepidoptera via van Leeuwen to J. Th. Oudemans).

508 Lodewijk Napoleon, Ajaccio 4. IX. 1778-24. VII. 1846. King of Holland I 806 -'Io, bought I 808 Utrecht on the fair the circus Alpy. With this he began a menagerie first at Soestdijk, later at Haarlem (Paviljoen) then Hortus Botanicus Amsterdam. Alpy remained the manager, while Reinwardt (and G. Vrolik) had the supervision. It was sold i7. VII. I8Io (Witkamp I 888 p. $3^{16}$ ), some animals went to Paris (Stricker, in Zool. Garten XIX 1878 p. 380 ; L.oisel 1912 III p. 34 seq.).

509 Loendersloot, Gerrit. Physician, Swijndregt. Cabinet (Blankaart, preface of his: Schouburg der Rupsen etc., Amsterdam I688 (A)).

510 Loggen, N., Hilversum. Butterfliès. Lempke 1936 p. 239 R.

5 II Loo, Salomon van. Cabinet sold (Houttuijn I76I-'73, I3 p. I75).

512 Loo, "Het Kleine Loo" and "Het Groote Loo" were two country-houses on an estate of the Prince of Oranje, $\mathrm{I} \mathrm{km}$ east of 's Gravenhage, near the "Huis ten Bosch". Here "Mevrouw de Gouvernante", Widow of Stadhouder Willem IV, founded a menagerie. A. Vosmaer had the supervision (1770). It ended with the French invasion (1795), when the animals were sent to Paris. Ferrner r.9 10 p. 495 (v I759); Björnståhl 1782 V p. 368 (v 1775); Courtanvaux I768 p. $197-99$ (v 1767. d); Coyer 1775 p. 266 ( v I769 d); Percy I775 p. 39 (v I77I); Lynar I78I p. I29 (v I77I); Grimm I775 p. 293, 295-96 (v I774 d); Honoré I779 p. 68 (v I776); Sander I783 p. 603 (v I777); Titius I783 p. 213 (v I777); Forstęr.I868 p. 71 (v I790); Vosmaer, I766-I804; Pallas I766 2 P. II, 21, 68; (Boddaert I770), I767-'77; Loisel I912 II p. 29 seq., Witkamp I864 p. II ' 1872 p. IX; Evers, de menageriën op het Loo, in: Bijdr. Meded. Vereen. "Gelre" XV p. 533; Houel, Hist. Nat. des 2 éléphans male et femelle du Musée de Paris, Io3 (A); W. de. Vink; in: Die Haghe I903, p. 355-59.

513 Loo, "het Oude Loo" near Apeldoorn in Gelderland. This was the oldest 
menagerie of the Princes of Oranje, begun as a "faisanderie" after Stadhouder Willem III had built a palace where there had only been a small "jachtslot". On the ups and downs see Evers, G. A. De menagerieën op het Loo, in: Bijdragen en Mededeelingen der Vereen. "Gelre" XV p. 533-540. It ended like het Kleine Loo with the French invasion in I795, when all of value was brought to Paris. Witkamp I872 p. IX. Loisel I9I2 II p. 29 seq. III p. 36; Uffenbach I754 II p. 374 (v i7Io d); Shaw I709 p. Io (v I700); Thouin I84I p. 255 (v I795 d). See also Vosmaer in his Règne Animal (e.g. I783 Afrikaansche eland p. 5-6); Allamand in his Dutch edition of Buffon; Kanold 1727 p. 65 ; Houel, Hist. nat. des 2 éléphans male et femelle du Musée de Paris, I803 (A).

5 I4 Loosjes, F. E., Harderwijk. Shells R.

515 Loovers, Jan DE. Middelburg. Cabinet of shells. Valentijn 1726 III p. 563 , I754 p. 47.

5 I6 Lormier, W. Cabinet of stones sold 's Gravenhage I759 (Vosmaer I790).

517 Louis NapolÉon see Lodewijk Napoleon.

518 Lucassen, François Théodore. 1858-'93. Inisects. Horn \& Kahle 1935-'37 p. 369. (Coleoptera to Everts, van der Hoop, Veth, Valck Lucassen, Mus. Leiden).

519 LuCassen see Valck Lucassen.

520 Luchtmans, Prof. Dr. P., Leiden I. IV. I733-Utrecht 2. II. I800. Prof. med. Utrecht. Cabinet of insects (Argenville I780 p. 337 ; Schaeffer I794 p. 136 (v I787-'88); Voet I766 I p. 28, 29, 53, 78, 79, 98, II p. 43, $5^{2}$; Cramer I779-'82 I p. 95, 99, I04, 120, I26, II p. 22, 24, 25, 30, 45, 46, 5I, 54, 86, 93, 98, 99, 126. (M. S. in UBA H. S. Cat. II n ${ }^{\circ}$ i 400). Van der Aa $1852-78$ VIII (b). Molhuijsen I9II-'37 IV Kol. 936 (b).

52 I LÜCKer, E., Roermond. Insects. Lempke 1936 p. 239.

522 Luder, Joannes, $\dagger$ r719. Amsterdam. Cabinet of shells. Valentijn i726 III p. 56I (after his death his cabinet went to his sons Arnold and Henrik). In 1720 however it was sold (Vosmaer I790). Valentijn I754 p. 25, 45, $48,53,58$.

523 Lufneu, H., Rotterdam. Physician. Cabinet. Valentijn I726 III p. 562, I754 p. 46. It was sold Rotterdam I5. III. I745. (Catalogue in B $\mathbf{n}^{\circ} \mathbf{1 6 6}$ p. 172-I90; Vosmaer 1790).

524 Luijck, Abraham van, born 1874, Amsterdam, later Baarn. Coleoptera. Everts 1922 p. XII ; Horn \& Kahle 1935-'37 p. 162, 370 (to Mac Gillavry).

525 Luijcx Massis, Daniël. "Hooftparticipants-Bewindhebber van de WestIndische Cie ter Kamer Zeeland". Middelburg. Cabinet sold 8. IV. I77 I (B $n^{\prime} 376$; Lugt $1938 n^{\circ}$ 1914). To him Gronovius dedicated the 2nd part of his Zoophylacium.

526 Luiscius, I. Rotterdam. Cabinet sold i780 (Vosmaer 1790).

527 Luther. Amsterdam. "Kunstdreher". Shells etc. (Uffenbach I754 III p. 542, v I7I0). 
528 Lycklama Á Nijeholt, Jacob Hector. 1874-i933. Butterflies. Lempke 1936 p. 239. Horn \& Kahle I935-'37 p. I62 (Lepidoptera to Zoöl. Mus. Amsterdam.

529 Lyonet, Pieter, Maastricht, 21. VI. I706-'s Gravenhage Io. I. I789. "Sécrétaire des Chiffres de L. H. P. les Seigneurs Etats Généraux des Provinces Unies etc." He is especially well known for his: Traité anatomique de la Chenille qui ronge le bois de saule (1760), of which he engraved the plates himself, like he did it for Trembley's work on Hydra (1744). M. S. in Hist. Natuurw. Mus. Leiden; Microscope in A (cf. Lyonet, in : Verhand. Holl. Mij (I) III p. 378, 4I3); (vide etiam: Handel. Entom. Vereen. I I p. 38, 42, 59; Tijds. Entom. Vereen. IV p. I4, VII p. 19, VIII p. 2 ; Konst- en Letterbode I86I p. 194, 393) also M. S. and plates of his posthumous work edited by de Haan (1832). He had a large cabinet of shells, insects and other naturalia, also pictures. Van der Aa 1852-'78 VIII (b) ; Hublard I9Io (b) ; Vosmaer I800 p. 347 n $^{\circ}$ I64, p. $350 n^{\circ} 25^{2}$, p. $356 \mathrm{n}^{\circ} 470$. He translated Lesser's 'Testaceotheologia (la Haye I742); Lieftinck 1925 in Tijds. v. Entom. LXVIII p. 63; Wheeler, 1926,The Nat. Hist. of Ants. p. 125 (letter of Lyonet to Réaumur); p. 26r (note 154); Collot d'Escury 1844 p. 189, 192, 201-7; Van Gool I750-I II p. 278, 330; Snellen van Vollenhoven, in Album der Natuur I880 p. I-I4; Lamy $193^{\circ}$ p. 28; J. de Conch. 74, I930 p. 49 note; Lesser I756 p. 73; Beckmann I9I2 (v I762); Thunberg I792-'94 II p. 260 (v I779); Honoré I779 p. 6I (v. I776); Forster I868 p. 68 ( I 1790); Anonymus I792 p. I09 (v I790); Smith I796 I p. 27 (v I786); Titius I783 p. 214 (v I777); Pallas I767-'77 X p. 33 ; Schaeffer I794 p. II2 (v I787-'88); Houttuijn I76I-'73, I3 p. I75-6, 16 p. 57, 537 ; Anonymus I767 p. I I3, 326; Sander 1783 I p. 493, 501 (v I776 d); Argenville I780 p. 343-5; I757 p. I48; Björnståhl I782 V p. 366 (v I774); Forster I 8433 p. 89 (v I778). His cabinet was first offered for sale in one lot for $£ 2000$ till $\mathrm{r}$. XI. I789 (A) by the executors of the testament Mr. van der Meersch and Mr. Croiset (heir) (vide Bibl. d. Sc. 1760 Oct.-Dec.). Then it was publicly sold in the Hague 21. IV. 1796 seq. (Catalogue in $\mathbf{A}$ n $^{\circ}$ 3258, Murray I904 II p. 36r, Lugt I938 $\mathrm{n}^{\circ}$ 5440). See also Tobias. Molhuijsen I9I I-'37 VIII Kol. Iogo (b).

$530 \mathrm{M}^{* * *}$, le Baron DE. Cabinet of fossils sold 28. X. I748 (Lugt $1938 \mathrm{n}^{\circ} 690$ ). 53 I MaAden, H. van der. Katwijk aan Zee. Shells. R.

532 MaAR, F. E. R. DE, 's Gravenhage. Shells. R.

533 MaARen, I. A. VAN. Cabinet (animals in liquor) sold 's Gravenhage I74I (Vosmaer 1790).

534 MaAstricht, Natuurhistorisch Museum (Musea 1938 p. 213 n $^{\circ}$ I53). Lempke 1936 p. 239. The vicinity of the St. Pietersberg stimulated the collecting of fossils in this town.

535 Maatschappij, Hollandsche, see Haarlem. 
536 MACARÉ see Rethaan.

537 Mac Gillavry, Dr Donald, born 1869 Surgeon. Amsterdam, now Bergen (N. H.). Insects. Horn \& Kahle 1935-'37 p. 162; Lieftinck 1925 p. 66-67; Everts 1922 p. XII. His son Dr Henry James (born 1908) collected in the West Indies and his daughter Miss M. E. collects also (Lempke 1936 p. 239).

$53^{8}$ Maitland, R. T. † 8. XII. I904. Zoologist, conservator Zoological Gardens Amsterdam 1854-'64 and again I880-'93, in the time between Director Zoological Gardens The Hague.

539 Mayer, Amsterdam. Merchant. Cabinet of shells (Anonymus 1767 p. 326). Is this the same as Meijer?

540 Man, Dr. Jan Cornelis DE, $t$. Middelburg 2. I. 1909. Physician. Archief Zeeuwsch Genootschap 19!o (b), I9I5 (b) ; Gedenkboek I9I9 p. 27 (portrait), I07 seq.; Nagtglas ' $1888-93$ III p. I2I-4; he iunctioned as conservator of the Zeeuwsch Genootschap 1859-'o9; his collection of Butterflies was given to Zoological Gardens Amsterdam (Gids voor de bezoekers Natura Artis Magistra 1864 in A, Blasius 1880 p. 13). Molhuijsen r9I I-'37 II Kol. 864 (b).

54I MAN, Johannes Govertus DE, son of preceding, Middelburg 2. V. 1850Middelburg 19. I. I930. Conservator Museum Nat. Hist. Leiden (Blasius I880 p. $19 \mathrm{~V}$ 1879), later living in Yerseke. His collections partly to Zeeuwsch Genootschap, of which he was a conservator '1909-'i8 (Gedenkb. I9I9 p. I08), partly to Zool. Museum Amsterdam; G p. 274.

542 MaN, Mr. M. J. DE. Nijmegen. His collection of butterflies was given by his heirs to Zoological Gardens Amsterdam (Maitland, in: Tijdschr. Dierk. I p. LV).

543 Mandere, Johanna Suzanna van Der, widow of Mr Johannes Gualterus van der Poort d'Oostcapelle. Middelburg (Lange Noordstraat). Sale catalogue 7-8. V. 1795 in $B n^{\circ} 633$.

544 Marcus, Paul. Amsterdam. "Materialiste", Lesser 1744 p. 54, 1756, p. 73. Son in law of Seba: (who had a son in law named Jacob Marcus, but not Paul, see Engel in Svenska Linné-Sålsk. Årsskrift. XX (I937 p. 86)

545 Marets, Des. 's Gravesande. Cabinet. Uffenbach I754 III p. 373 (v 1710), see Desmarets.

546 MARK, van DER, "Baljuw te Warmondt", married the widow of L. Vincent (see there).

547 Marle, J. G. van, Aerdenhout. Collection of palaearctic birds together with Sillem (bought from Snouckaert). $R$.

548 Marre, Evert DE, Amsterdam, "Administrateur van het Ouderzydshuyszittenhuys". Cabinet of insects. Argenville I780 p. 355; Cramer I779-'82 I p. $36,38,52,65,124,137$, II p. 82 , I I3, IV p. I73, I76, I77, I80 (C. de Marre on p. 202), 21 I, 212, 235, 238, Appendix p. I73; Stoll 1788 1 p. 79, 80,8 r, 165, I788 2 p. 55 ; Nemnich I809 p. 37r (v I808). 
549 Martens, Jonkheer Jacob Carel. Utrecht 19. XII. 1817 - de Meern 2. VII. 1872. Cabinet of insects, shells, birds, which was given to Zoological Gardens Amsterdam (Maitland, Ned. Tijdschr. v. d. Dierk. I r863).

550 Martin, P. Henri, Marseille io. I. I793-Rotterdam-?-. "Dompteur". He sold his animals to Van Aken, who again sold them to the Zoological Gardens Amsterdam ( 1838 , Jaarboekje Natura Artis Magistra 1852 p. I 14). In I 836 a curious booklet was published : Geschiedkundige levensschets van den Heer Martin..., 's Gravenhage (van de Lyon).(A nº 222), and also: Beschrijving der dieren, welke zich bevinden in de menagerie

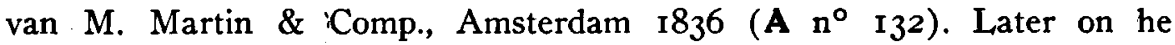
became Director of the Rotterdam Diergaarde (1857-66). Lax I838 p. 122-5 (v 1837); Witkamp I872 p. XXV; Loisel I9I2 III p. 307, 305 (Pichot, Les mémoires d'un dompteur, Paris I88I, p. IIO, I30). Life also in : Rotterdamsche Diérgaarde, Rotterdam 1858 , p. $49-52$ (A).

551 Martinet, Ds. Joannes Florentinus. Deurne 12. VII. 1729-4. VIII. I795. Clergyman, Dr. phil., his dissertation being: De respiratione insectorum (1753); Levensbericht by A. van den Berg. Amsterdam i726 (b); Collot d'Escury I844 VII p. 215-220; Harting, in Album der Natuur I883 p. I (b); Van der Aa 1852-'78 VIII (b). Eyndhoven I86I $n^{\circ}$ 123; Well known is his "Katechismus der Natuur" Amsterdam I779-82; Ehrhart 1783 p. 213 (v I782).

552 Martini, Dr. A. A. Physician in 's Hertogenbosch. His collections were sold 27-28. X. I778 (B n ${ }^{\circ} 440$ p. 56).

553 Marum, Dr Martinus van. Delft 20. III. 1750-26. XII. I837 Haarlem. Dr. med., Director of the Cabinet of Natural History of the Hollandsche Maatschappij der Wetenschappen at Haarlem (1777). He seems to have had also collections of his own, though this never is quite certain because he lived in the Museum of the Hollandsche Maatschappij: In I794 he became Secretary to the Holl. Mij. and 1815-'20 he also assisted Temminck in the direction of the Cabinet du Roi in Amsterdam. In I784 he became also Director of the Cabinet of Physics and Nat. Hist. (fossils) of Teyler's Genootschap. Sander. I783 I p. 54I (v 1776); Eversman 1792 p. 79 (v 1783); Smith I796 p. 23 (v I786); Schaeffer I794 p. I24 (v I788); Forster I868 p. 98,99 (v I790); Anonymus I792 p. 77 (v I790); Thouin I84I p. 235 (v I795); Sierstorpff I804 p. 55I, 555 (v I802); Niemeyer I823 p. I5I, I52 (v I806); Nemnich I809 p. 374 (v I808); Haupt I8I4 II p. 192-8 (v 18Io); Meiszner I8I9 p. 72 (v I8I7); Strick I8I8 p. 154 (v I8I7); Neill I823 p. I88 (v I8I7); Fliedner I831 p. 286, 289, 394 (v I823-'24); Jaeck I824 p. 225 (v 1824); Dethmar I838-4I p. I6I (v i835-37); Sepp i762-I860 III p. 66; Muntendam I938 p. 464-493 (b) ; Veen in Ned. Tijdschr. v. Geneesk. 82, 1938 p. 42-66 (b) ; Witkamp I 888 p. 306-8; Camper I88I p. I5 (letter. M. S.); Molhuysen I9I I-'37 X Kol. 588 (b) ; Cohen, I938, Proc. K. Ned. Akad. Wet. Amsterdam, XII p. 246. 
554 MatzedelaAR, Johannes. Cabinet sold 9-Io. III. I792 Leiden (Catalogue in $\mathbf{A})$.

555 MaUritz, Mr. Johannes. Collection given to Zeeuwsch Genootschap 25. VI. I77I (Gedenkboek I9I9 p. IOI).

556 Medenbach de Rooy, Dr. med. Nijkerk. Collection of insects. Sepp. 1762-1860 VII p. IV, 37, 50, 71, 76, 77, 121, 133, 150. Catalogue in Ontyd's Catalogue (6. XI. 1878 Amsterdam in E).

557 Meder, J. C., "Secrétaire de la Banque de Java". † I845. Cabinet of shells sold at Paris 1852 (Catalogue in Bibl. Dautzenberg now in Mus. Roy. d'Hist. Nat. Brussels $n^{\circ}$ 52352). Kalff I928 p. 205-6.

$55^{8}$ Meentzen, Johan Adolf. "Gewezen schepen tot Batavia". † Enkhuizen. Cabinet of shells which he took I7I4 to Holland. Valentijn I726 III p. 56I, I754 p. 45.

559 Meer, Ter. Leiden. Insects (Sepp. 1762-I860 VIII p. 8).

560 Meer, G. van Der, born I6. XÍI. I89i, now Eefde, Secundary teacher in Zutphen. Collection of Dutch birds and eggs. $\mathbf{R}$.

56I Meer, D. van Der. Delft. Cabinet. Valentinus I7I4 II p. 19; Kanold I727 p. I95.

562 Meerburgh, Nicolaas. Hortulanus Leiden. Cabinet (Sander I783 I p. 5 I8, v 1776$)$.

563 Meijer (see also Maijer). Some Meijer's could not be traced, so: Francq 1773 p. 35 .

564 Meyer, "Secrétaire de la Chambre des Etats à Utrecht". Argenville I757 p. 149; I780 p. 336 (sold at his death).

565 Mexer, Christianus Paulus. Merchant. Amsterdam, later Utrecht, Argenville 1780 p. 354 ; Pallas I766, p. I80, 262, 286; Boddaert 1768 p. XX, 224, 326, 355; Houttuyn I76I-'73 I7 p. 121, 174, 178, I83, 279, 319, 330, 351 ; Vosmaer I800 p. $35^{1} \mathrm{n}^{\circ} 286$ (earlier Amsterdam, now Utrecht); Sander I783 I p. 565 ( $v$ r777). Cabinet sold $4 . \mathrm{X}$. I802 seq. in Utrecht (Catalogue in $E$ and Libr. Dautzenberg in Mus. Roy. d'Hist. Nat. Bruxelles $\left.n^{\circ} 5^{2367}\right)$.

566 Meyer, Cornelis. Amsterdam. Cabinet: Boccone I674 p. 278 (v I673-'74).

567 Meyer, C. G. Cabinet of divers naturalia sold i780 Amsterdam (Vosmaer 1790).

568 Meyer, Johan Frederik, "Boekhouder op Amboina". Valentijn 1726 III p. 560,1754 p. 44 (did he return in Holland?).

569 Meyer, J. Gotlieb. Amsterdam. Merchant. Shells. Argenville i780 p. 354.

570 Meijere, Prof. Dr Johannes Cornelis Hendrik DE, born Deventer I. IV. I866. Professor of Entomology Amsterdam. Collects Diptera. Presented his collection to the Amsterdam Zoological Museum in 1935. Gedenkboek I932 p. 636, 277, 278, 279, 285; Horn \& Kahle 1935-'37 p. I7I; Everts I922 p. XII. R.

571 Mere, Jan van Der. Druggist. Delft. Ray i738 II p. 24 (according to Murray $1904 \mathrm{I}$ p. 60). 
572 Merian, Maria Sybilla. Frankfurt a. M. 12. IV. 1647-I3. I. I7I7 Amsterdam. Paintress who travelled to Surinam to paint insects. Chemnitz r76o p. 74; Collot d'Escury 1844 VII p. I99-200; Uffenbach I754 III p. $55^{2}$ (v I7Io); Maitland I855 p. I68; Horn \& Kahle 1935-'37 p. 173. (Collection to St. Petersburg, ex parte to Wiesbaden); Argenville I757 p. I74, I780 p. 336. Van der Aa 1852-'78 VIII (b); Molhuysen I9II-'37 VIII Kol. I 46 (b).

573 MessedalaAr, see following.

574 Messedaler, Druggist. Rotterdam. Cabinet (Argenville I780 p. 338). Anonymus 1767 p. 327 speaks of cabinet of shells of M. Messedalaar, apothicaire à Rotterdam.

575 Meulen, G. S. A. van der. Amsterdam. Lepidoptera. Lempke i936 p. 239. R.

576 Meulen, Jan Ter, Amsterdam, Insurer. Collection of birds, which he later gave to Zoological Museum Amsterdam.

577 Meulen, Willem van Der. Amsterdam. "Regent O. Z. Huijszittenhuijs, Capitein eener Burgercompagnie". Large cabinet of shells, insects, stones etc. As a Director of the Zeeuwsch Genootschap he gifted it with shells (Gedenkboek I9I9 p. I04)، He gave a collection of stones to the Botanical Garden in Amsterdam (Wagenaar, J., Amsterdam in zijn opkomst. II I765 p. 387). Francq I773 p. 35 ; Argenville I757 p. I49, I780 p. 350-5I (d); Anonymus 1767 p. 328 (Vandermeulen); Stoll I $788_{1}$ p. 83,87 ; Cramer I779-'82 I p. $36,37,57,64$, I23, I37, I39, I40, I48, II p. 23, 40, 46, 73, 89, IO3, I2I, I24, I39, III p. 38, 40,48, 50, 53, 74, 80, 87, 88, I I2, I I3, I I8, I 20, 126, 127, I35, I 39, I 43, I 58, I62, IV p. 22, 25, 30, 32, 35, 38, 40, 46,

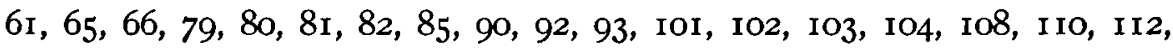
122, I23, I25, I29, I32, I34, I37, I43, I44, I46, I48, I49, I58, I59, I63, I67, I83, I85, I86, I90, 209, 215, 219, 230, 234; Van Phelsum I774 p. 59; Pallas $17666_{1}$ p. 55, I80, 283, 286, 386; Boddaert I768 p. XXIV, 68, 224, 35I, 355, 487; I770 V p. 27; Knorr I770-'75 V pl. I; Houttuijn I76I-'73, 8 p. 276 (p. 20 last part), 9, p. I77, I79, 53I, 534, 542, 549, I2 p. 24I, I 4 p. 448, I 7 p. I I7, I2I, I45, I63, I74, 330, 346, 433. His garden seems to have shown a menagerie: Camper (Oeuvres, I803, I p. 50) saw there in 1772 an orang-outan (Loisel 1912 II p. 50). His Cabinet was sold I782 Amsterdam (Vosmaer 1790, who also, under F, mentions Amsterdam 176I divers naturalia Ferguson van der Meulen); Sander I783 I p. 568 (v I777, writes van der Moelen); Titius I783 I p. I95 (v I777, van der Moelen).

578 Meulen, see also Mollem, Termeulen, Vermeulen.

579 Meuschen, Friedrich Christian I719-about I800. "Legationsrath". He made catalogues of shells for auctions, collecting them in a series: Miscellanea Conchyliologica (they were also issued separate) by F. C. M. See I Chais, 2 Mieden, 3, Oudaan, 4 Leers, 5 Dishoek, 6 Koening, 7 Zuylen van Nyevelt, 8 Gronovius, also Gevers, Schwenken. He is mentioned in Pallas 1767-'77 IX p. I4, 26 (insects!); Anonymus 1767 p. 327 (shells). His signature in album amicorum F. Ruysch II (UBA). 
580 Michielsgestel, St.,. (N. Br.). Natuurhistorisch Museum van het R. K Seminarie Beekvliet (Musea 1938 p. 218 n $^{\circ}$ I59) $\mathbf{R}$.

58I Middelburg. In earlier days animals were kept in the townhall (eagle, Payen 1663 p. $42 v \pm$ I660; Houttuyn I76I-'73 I 4 p. 127). Here the Zeeuwsch Genootschap der Wetenschappen (founded Vlissingen I769 (I765), I797 to Middelburg, Museum I80I) has its seat and Museum (Vrolik in: Versl. Meded. Kon. Akad. Amsterdam. Afd. Natuurk. V.p.'16; Gedenkboek 1919; Terwen I855 p. 376; Musea I938 p. 221 $n^{\circ}$ I64). I may refer to the Gedenkboek for further information and mention only Fliedner: 1831 p: 394 (v 1823-'24).

582 Mieden, van DER. Amsterdam, "Raadsheer". Catalogue of sale by Meuschen 30. IV. I766 (A $3126 ;$ Vosmaer 1790, divers naturalia).

583 Mieris, van, probably Willem; I662-i747 Leiden. Painter. Cabinet. Uffenbach 1754 III p. 423-4 (v I7IO).

584 Milan, Gisbertus Franc de Milan Visconti, Liber Baro etc. "Secretaris van de Staten van Utrecht." Argenville I780 p. 337. His cabinet of nat. hist. was sold Utrecht 4. XI. I782 (B n $\left.{ }^{\circ} 464\right)$; Lugt I938 $\mathrm{n}^{\circ} 3473$.

585 Mieropius. Uffenbach I754 III p. 394-95 (v I7IO). Probably Nieropius.

586 Moelen, van Der, see Meulen.

587 Moerbeek, Dr. Abraham van. Physician Amsterdam. His Sale-Catalogue 20-21. XI. 1788, Amsterdam (B $\mathrm{n}^{\circ}$ 524) mentions some anatomical praeparations and animals.

588 Molengraaff, G. A. F. Butterflies of Holland about I900 to J. Th. Oudemans (Horn \& Kahle I935-'37 p. 18I).

589 Mollem, van. “Zydebalen”, Silk manufactory near Utrecht. The owner's garden was from 1720 well known for its curiosities, grottoes etc. See S. Muller, Zydebalen. Utrecht I9I2, $4^{\circ}$ (UBA). Moreover he had a cabinet of shells etc. Haller I 883 p. 56, 6i (v I725-'26) ; Biema i9ro p. 90 (v I736); Poole I743 II p. 87 (v I74I); Garampi I889 p. I84 (v I762); I899 p. 218-9 (v I764); Anonymus I790 p. I $47-8$ (v I784); Seba I734-'65 III p. II2: Some authors have written Van der Meulen. See also Evelyn I879 p. 247 (visited I64I a silk manufactury of Mr. Seterveldt with an "old fashioned" menagerie).

590 Molm, see van Mollem.

591 Muilman. Dr. 's Gravenhage. Cabinet with animals in liquor sold 1789 (Vosmaer I790). A son in law of Seba was called Muilman (Engel 1937 Sv. Linné-Sålsk. Årsskr. XX p. 87).

592 Mulder, Prof. Dr. Claes. Leeuwarden 6. X. I796-Groningen 4. V. I867. Prof. chem. Franeker (1822) and Groningen (184I) after van Swinderen's death ( $185^{2}$ ) also Nat. Hist. (till 1866 ). His collection were bought I $84 \mathrm{I}$ by the University Museum Groningen (List edited by Mus. Groningen in A). Dethmar I838-'4r p. I87 (v I838); Van der Aa I852-'78 VIII (b). 
593 Muller, J. R. Amsterdam. Shells. R.

594 Muller, Leo, born 1898. Secondary teacher. Bussum. Shells R.

595 Muller, S. Cabinet bought by Lidth de Jeude (see Lidth de Jeude 1858 preface).

596 Nahuijs, Prof. Dr. Alexander Petrus. Monnikendam Io. I. 1737-Utrecht 6. IV. 1794. Prof. med. Harderwijk (I77I), Utrecht (1775). His cabinet especially stones and metals was sold $\mathrm{I} 6$. VI. 1800 in Utrecht (B $\left.\mathbf{n}^{\circ 1} 678 \mathrm{II}\right)$. Schaeffer I794 p. I36 (v I788); Ehrhart I783 p. 224 (v I782). Van der Aa I852-'78 IX (b).

597 Napoleon, see Lodewijk.

598 Neervoort van de Poll, Jacob R. H., I862-1925. Coleoptera. Horn \& Kahle r935-'37 p. 2 Ir.

599 Nereynen see Cocq van Nereynen.

600 Neuteboom, W., Heemskerk, Shells.R.

601 Neuville, Amsterdam. Banker. Cabinet (Argenville 1757 p. 149, 1780 p. 350 ).

602 Nierop, van (Nieropius, Mieropius). 's Gravenhage. Museum. Valentinus I714 II p. I9; Kanold I727 p. I98; Uffenbach I754 III p. 394-5 (v.1710, Mieropius).

603 Nies, C. Butterflies. R.

604 Nieurof, Amsterdam. Merchant. Argenville I780 p. 355, Valentijn I754 p. 9.

605 Nieuwhof, see Nieuhof.

606 Nieuwveldt. Rotterdam. "Secretaris van de Weeskamer". Cabinet sold I773 (Vosmaer I790).

607 Nijevelt, see Zuijlen.

608 Nijmegen. In this very old town the Dukes of Gelderland kept lions and other animals (parrots) (Witkamp 1872 p. V, Loisel I p. 156). Veryard I70I p. I6 ( $v$ I682 saw a monstrous child in the University). Haller I 883

- p. $5^{8}(v \pm \mathrm{I} 725)$. mentions the Reichswald.

609 NiJs, Isaac Jan. Boccone 1674 must have mentioned this man as a collector (Kerbert 1926 p. 3).

610 Nijvrelt. Catalogue mentioned by Cobres 1782 I p. I39, belongs to the Miscellanea of Meuschen (see there) $n^{\circ} .7:$ v. Z. v. N. = van Zuylen van Nijevelt, see there.

6II Nonnekens, A. C. Amsterdam. Coleoptera Everts I922 p. XII. R.

6i 2 Noortberg, J. Tergouw. "Echevin". Cabinet of minerals (Argenville I780 p. 358), sold 's Gravenhage 1765 (Vosmaer 1790 divers naturalia).

6 r 3 Nopf. Amsterdam. Cabinet (Argenville r780 p. 356).

6I 4 Nozeman, Ds. Cornelis. Amsterdam 15. VIII. I72I-Moordrecht 22. VII. I785. Clergyman in Alkmaar, Haarlem, Rotterdam (one of the founders - of the Holl. Mij. and Bataafsch Genootsch.). Van der Aa I852-'78 IX (b); Collot d'Escury I844 VII p. 243; Francq I769-I8I I, III p. I86; Maitland 
I855 p. I68; Sander I783 I p. 470 (v I777). Well known is the work on birds he edited with Sepp: Nozeman 1770. Molhuysen 1911-'37 X Kol. 678 (b).

$6{ }_{5}$ Nuwrel.t, Nyvrelt, Nuvrelt, see Zuijlen.

616 OdÉ, H., Delft. Shells. R.

617 Odon, Jacob. Cabinet of shells sold Amsterdam 6. IX. 1784 (Lugt 1938 $\left.n^{\circ} 3771\right)$.

618 Oets, Pieter. Amsterdam. Painter. Among the things from him and others sold Amsterdam 3I. I. I79I were 266 nrs nat. hist. (Lugt $1938 \mathrm{n}^{\circ} 4660$ ).

6rg Oltmans, Miss M. E. A. 's Gravenhage. Her shells came to following.

620 Oltmans, A., $\dagger$ 1874. His collection of shells (which he got from Miss Oltmans, see above) was bought by the Zoological Gardens Amsterdam I 844 (Jaarboekje Natura Artis Magistra I874 p. I88); Maitland in Ned. Tijdschr. v. d. Dierk. I 1863, p. LVI; Tijdschr. Ned. Dierk. Vereen. I p. I33. He became conservator of the Museums of the Zoological Gardens Amsterdam.

62 I OntiJd, Miss F. J. M., see Rethaan.

622 Ontijd, Dr. C. G. R. † I3. I. 1844 Brummen (68 years old). Physician. 's Gravenhage. Cabinet sold 6. XI. 1878 in Amsterdam (Catal. in E, contains also collection Medenbach de Rooy).

623 Oortman, A. Med. Dr. Cabinet, see Boddaert i768 p. XXIII.

624 Oortmans, Mrs. (Mej. or Juffr.) (Petronella de la Court, widow of Adam Oortmans). Amsterdam. Cabinet of shells. Rumphius 1705 p. 67, 86, 108, I33, I60; Valentijn I726 III p. 56I ; I754 p. 44, 48; Collot d'Escury I844 VII p. 329; Uffenbach I754 III p. 648, 9 (v I7IO).

625 Oortmans, Hendrik. Amsterdam. Cabinet of shells (Valentijn 1726 III p. 56r ; 1754 p. 45) sold Amsterdam I749 (Vosmaer 1790).

626 Oostcapel.le, see Mandere.

627 Oosterdijk Schacht, Prof. Dr. Hermannus. Amsterdam 8. VI. 1679Leiden 15. II. 1744. Prof. med. Leiden 1719. In the sale catalogue of his libray (B 162 ) is a collection of animals in liquore and sicco modo conserv. (p. 99-IOI). Van der Aa 1852--'78 IX (b); Suringar I867 Ned. Ts. v. Geneesk. (2) II 2 p. I99; Haller I883 p. 39, 107 (v I725).

628 Oosterdijk Schacht, Johannes, son of preceding. Leiden 26. X. I704Utrecht I8. VIII. I792. Prof. med. et philos. Franeker (I728), Utrecht ( $1729-1790)$. His collections (catalogue $B n^{\circ} 606$ ) contained especially minerals and microscopes of Leeuwenhoek (p. $201 \mathrm{n}^{\circ}$ 5). Schaeffer 1794 p. 136 ( $v$ I788).

629 Ooststroom, Dr S. J. van. Assistant Rijks Herbarium, Leiden. Coleoptera $\mathbf{R}$.

630 Oranje. The Princes of Oranje, Stadhouders of Holland (Maurits 15671625, Frederik Hendrik I584-1647, Willem II I626-'5o, Willem III 
I650-I702, Willem IV I7II-'5I, Willem V I748-i806) were all more or less interested in animals. They possessed a menagerie or a cabinet and the Officers of the East and West Indian Companies of fered them the curious animals the ships brought to Holland. In 1795 the French brought everything of importance to Paris. In 1813 , after the restoration of the Kingdom, King William I received back an adaequate collection, which Brugmans selected in Paris, gave his collections to the Leiden University where they formed the beginning of the Rijksmuseum van Natuurlijke Historie (see also G p. IO, 23-28). The Amsterdam zoological gardens have enjoyed the interest and protection of our Kings William I, II, III and of Queen Wilhelmina. There is still a collection in the possession of the House of Oranje and much about the old ones can yet be found in the Huisarchief. While I have dealt with the menageries s. v. Honselaersdijk, Loo, Soestdijk, I here only mention the cabinet of shells of Prince Maurits (Brom I9r 5 p. 126 v 1677-78) and the "Kabinet des Stadhouders". It was founded I756 (Argenville I780 p. 338) by the Widow of Willem IV, "Mevrouw de Gouvernante", who bought Vosmaer's cabinet and made him director of it, later on the cabinet Lassara and others were bought. The Cabinet was situated since Sept. 1766 in the N. E. edge of the Buitenhof, opposite the Palace of the Stadhouders. (Die Haghe I933 p. 54 seq., I22; Oud Holland X I892 note I p. 224). Vosmaer I 800 p. $34 \mathrm{n}^{\circ}$. I 53 mentions a catalogue, perhaps M. S., made 1778 for the Princess. As is well known he described many animals in Vosmaer $1766-1804$ of which many passed from the menagerie in the cabinet. He probably had a clerk at his disposition named G. C. Blanken, who figures as subscriber in a book of the time. Pallas of ten studied in menagerie and cabinet; Pallas $1766_{1}$ p. XV, $45,46,64,88,158,168,179$, I81, 190, 216, 219, 232, 326, 346, 350, 370; Boddaert 1768 p. XIX, XXXVI, 55, 57, 78, 108, 198, 210, 223, 225, 236, 270, 274, 290, 403, 429, 435, 46I ; Pallas I 766 2 p. 36, 56, 57, 6I-64, го6; Boddaert I770 I p. VII, VIII, I5, 21, 31, V p. 27, 29; Pallas 1767-'77 I p. $16,23,38$, II p. 5, VI p. II, I8, I9, 33; see also: Houttuijn 1761-'73, 17, p. 249, 397; Cramer I779-82 I p. 139, II p. I, 19, 39, 57, 58, 60, 77, 9I, III p. I0, 41, 54, 63, 8I, I36, I57, I64, I65, IV p. I3, I42, I45, 150, I5I, I8I ; Temminck I808-'9 VI p. 49; Voet I766 I p. 6, 10, II, 19, 20, 2I, 23, 30, 31, 40, 48, 53, 77; Stoll $1788_{2}$ p. 99. Of course it was often visited and because of its richness described. Ferrner 1910 p. 352 (v I759); Garampi I889 p. 204 (v I762) ; I899 p. 2I4, 266 (v I764); Beckmann I912 p. 344 (v I762 d); Courtanvaux I768 p. 19I-2 (v I767 d); Argenville I780 p. 338 (v I773 d); Heinecken I769 p. 64 (v I768); Coyer I775 p. 266 (v I769); Thunberg I792-'94 II p. 260 (v I779); Lynar I78I p. I3I (v I77I); Björnståhl I782 p. 367-8 (v I774); Grimm I775 p. 290 (v 1774 d); Sander I783 p. 487 (v I777); Maarsen in: die Haghe Jaarb. I936 p. 269 (v I778); Silva Rosa 1927 p. I8 (v I778); Honoré 1779 p. 59 
(v 1776); Titius 1783 p. 204-213 (v I777 d); Desjobert I910 LIX p. 8 (non v I778); Eversman I792 p. I88-I9I (v 1783 d); Smith 1796 I p. 27 (v 1786); Schaeffer I794 p. I IO-II2 (v I788 d); Ireland I790 p. 70 (v.r789 d); Forster I868 p. 69 (v I790); Anonymus I792 p. IOI (v I790 d); Radcliffe 1795 p. 65 (v 1794). See also Anonymus 1767 p. 327 (m); Denys de Montfort I I808; Stresemann 1923 p. 124; Van der Klaauw 1926 p. 8, 59, 60, 6I ; Horn \& Kahle I935-'37 p. 267, 383; Maitland I855 p. 169; Witkamp 1888 p. 313. About the collections in Paris see Cuvier \& Valenciennes, Hist. Nat. des Paissons, I828-'49, XIII p. 304 etc.; Proc. Zool. Soc. 1892 p. 317. When the cabinet selected by Brugmans was in the Hague but not yet given to the Leiden University it was visited by Strick I8I8 p. 197 (v I8I7); Jaeck I826 p. 212 (v I824).

63I Orsoy, Jan Arnold van, Amsterdam ("op de IJ-gracht, bij de Peperstraat"). Cabinet of anatomy, insects etc. sold I9. I1. I754 Amsterdam (B 223a).

632 Orville, Ds Wilhelm D', Hamburg i651 or I652-Amsterdam 31. III. I719. Clergyman Vlaardingen ( I678) Haarlem (I $700-$ o7). Molhuijsen I9I I'37 V Kol. 407 (b) : Valentijn I726'III p. 562, I754 p. 46, 50, 5I, 65; Lesser I756 p. 78 (m. catalogue I722); Uffenbach I754 III p. 509 (v 1710); Seba 1734-'65 II p. 134. The cabinet (shells, zoophytes, stones etc.) was sold

'. I722 Amsterdam. (Boehmer I785 I p. 375 who wrongly writes I622; he mentions a second edition of I736); Molhuijsen I9I I-'37 V Kol. 407 (b).

633 Otweiler Cabinet sold Amsterdam i766 (divers naturalia) (Vosmaer 1790).

634 Oudan, Michiel. Rotterdam \pm I702-30. V. I766 merchant. He seems to have been a nephew of Houttuijn, who I76I-'73, I2 p. 2I speaks of a drawing with 3 Odonata, by Rocho van Veen I68I, he got from-his nephew Oudaan (these are now in E). His cabinet of shells was catalogued by Meuschen (Miscellanea 3) and sold 18. XI. I766 Rotterdam (A; Murray I904 III p. 69; Vosmaer I790; Lugt 1938 n $^{\circ}$ I 562): Nederl. Leeuw XLIX I93I Kol. I43.

635 Oudemans, Dr. Anthonie Cornelis. born 1858. Secondary teacher in Arnhem. Collects Acarina. Everts 1922 p. XII ; Wulp 1895 p. 20 . R.

636 Oudemans, Dr Johannes Theodorus. Amsterdam 22. XI. I862-Putten 20. II. 1934. Some time conservator Zoological Museum Amsterdam (I8921902). During many years President Dutch Entomol. Soc. A great collector of Dutch insects especially Lepidoptera and Hymenoptera, which after his death came to Zoöl. Museum Amsterdam. Biography by Max Weber in Tijdschr. v. Entomologie Suppl. vol. LXXV, 1932 p. I, see also ibid. LXXVII I934, p. I67; Gedenkboek 1932 p. 279; Wulp I895 p. 50, 89; Everts 1922 p. XII ; Lempke I936 p. 239 ; Horn \& Kahle I935-'37 p. I99.

637 Oudshoorn, Jan Gosche van. Cabinet sold io. IV. i787 Amsterdam (Lugt I938 n $^{\circ}$ 4170; Vosmaer 1790).

638 Oukerke, Laurens van. Haarlem. 25. IV. 1748--buried 23. VI. I817. Married Geertruijda van der Vinne. Cabinet (Argenville I780 p. 357 Ovkerke); 
Nemnich I809 p. 37 I v'I808, insects (Laurens or Willem, following,?); Ekama I874 p. I03 (sold I9. V. I818 Haarlem, catalogue in B).

639 Oukerke, Willem van, Haarlem. 25. VII. I744-I6. VI. I795. Married Christina van der Vinne. Cabinet (Argenville I780 p. 357 : Ovkerke) ; Nemnich I809 p. 37I, v I808, insects (Willem or Laurens, preceding?) ; Ekama I874 p. Io3 (sold Haarlem I I. III. I8I6, also Lugt 1938 n $^{\circ} 883 \mathrm{I}$ ).

640 Ouwens, Prof. Dr. Wilhelmus. Delft I7I7-'s Gravenhage I5. III. I779. Prof. med. Franeker (I749-'63). 's Gravenhage, Cabinet sold I777. (Vosmaer I790). Van der Aa I852-'78, IX.

64I Ovens, Amsterdam, Painter. Cabinet of Shells Rumphius I705 p. Io8, I6I; Valentijn 1726 III p. 56r; 1754 p. I9, 45, The cabinet was sent to England after his death, to be sold there (Rumphius l.c.).

642 Padbrugge, see Hoogeveen.

643 Palier, J. C. 's Hertogenbosch. Professor and clergyman. Cabinet of divers naturalia, shells, fossils, corals sold: 27. V. I782 (B $\left.\mathrm{n}^{\circ} 459\right)$.

644 Paludanus, Bernardus. (Berent ten Broeke) I550-Enkhuizen I633. Physician in Enkhuizen. Hunger in Ned. Tijds. v. Geneesk. $72,1928{ }_{2}, \mathrm{n}^{\circ} 44$ (b); Sincerus r649 p. 237 ( $v \pm 1610$ ); Hegenitius 1630 p. $5^{8}$ ( $v$ before I627, d of cabinet) ; Rathgeb, J., Warhaffte Beschr: zweier Raysen... (with Index rerum omnium naturalium a Bernhardo Paludano... collectarum), $\because$ Tubingen, 1603 (UBA); Jacobaeus 1910 p. 77 (v Enkhuizen I674 and remembers Paludanus); Monconys I666 II p. I75 (v Enkhuizen I663). ("Nous n'y pûmes voir... le cabinet de Paludanus, parcequ'il n'y avoit que 3 semaines que M. Stemberque à qui il appartenoit, étoit aussi mort, et toutes choses estoient encore sous le seau"). This refers to what remained of the collection after part of it had been sold in 165 I to Duke Friedrich zu Schleswig-Holstein in Gottorf (see Olearius, Gottorfische Kunstkammer, Schleswig 1666 or 1674, Preface p. 5 A), later on this Kunstkammer went to St. Petersburg. Valentinus 17I4 II p. 19; Kanold I727 p. I95; Lesser I756 p. 79 (quotes Grotius, Poemat. 276); much literature gives Murray I904 I p. 95-6, III p. 208; Collot d'Escury 1844 VII p. 302; Molhuysen I9II-'37, IX Kol. $75^{2}$ (b).

645 Pama, Pieter. Cabinet of divers naturalia sold 30. I. I78 I Amsterdam (Lugt $1938 \mathrm{n}^{\circ}$ 3208).

646 Pancras, Mr. Gerbrant. Burgomaster of Amsterdam, Director East Indian Comp. Cabinet sold 20. III. I 726 (Valentijn I726 III p. 56I ; I754 p. I9, 45); Kalff I928 p. 207, 2 I0.

647 Patin, C., Amsterdam. Cabinet (Misson r722 I p. 34, v i687).

648 Pelt Lechiner, A. A. van. Arnhem. Birds eggs. Published in 2 yol..Oọlogia Neerlandica, the Hague, I9I0-'I3 (A). R.

649 Pereira, had a large aviarium on his estate near Maarsen (où on avait accouplé une poule et un lapin) (Desjobert I9Io LIX p. 340, v I778). 
650 Persijn. The house Persijn, near the Hague. Menagerie with rare birds. Ferrner I9IO p. 347 (v 1759).

651 Phelsum, Murk van. Med. Dr. Physican in Sneek. Describes animals from his own cabinet in van Phelsum 1774, p. 59.

652 Philips, Joan Caspar. Born before I700-about I765, and his brother George. Amsterdam. They induced Sepp to the collecting of insects (Sepp I762I860 I 3 p. 21, 4 p. 2). Joan Caspar was an engraver (Houttuyn I76r-'73, 9 p. I 47, 499, I3 p. 226, I4 p. 37) who worked for Houttuyn and had a cabinet himself; Wurzbach, Niederl. Künstler. Lex. II. I9Io. 'p. 326.

653 Pichart. Utrecht. Dr. Med. Cabinet of shells (Anonymus I767 p. 327, Argenville r780 p. 8I I).

654 Piepers, Mr. Marinus Cornelis, \pm 1836-1919. Lepidoptera from East Indies to Mus. Leiden (Horn \& Kahle I935-'37 p. 209); G p. I69.

655 Piet, D., Amsterdam. Lepidoptera. Lempke 1937 p. 245.

656 Pietterson, J. J. Cabinet of shells etc. sold 's Gravenhage 1769 (Vosmaer I790).

657 PinTo, David DE. $†$ I75I. His garden near Amsterdam was very beautiful, with grottoes etc. Weissmann in: Amsterdamsch Jaarboekje r888 p. I22; Seba 1734-'65 III p. II2; Haller I883 p. I03; v 1726; Biema I9Io p. 77-92 (v I736); Willebrandt I76I p. 90 (v \pm 1757); Björnståhl I782 p. 375 ( $\mathbf{v}$ I775).

658 Ploeger, Carel, Broek in Waterland "un des plus riches assuradeurs de Hollande" Collection of stuffed birds, minerals etc. (Desjobert I9IO, LIX p. I95, v I778).

659 Polak, R. A. Amsterdam. Teacher. Curator of Insectarium in the Zoological Gardens. Insects. R.

660 Poll, see Neervoort van de Poll.

66I Polvliet. Rotterdam, "iaissanderie" since I845 (Loisel i9I2 III p. 87).

662 Ponderus, Maria. Cabinet of shells sold 's Gravenhage i764 (Vosmaer I790).

663 Poort D'Oostcapelle, Johannes Gualterus van Der, see Mandere.

664 Porretr, Christiaan. Druggist. Lêiden. His cabinet of shells, minerals etc. sold Leiden 28. III. I628 (Lugt $1938 \mathrm{n}^{\circ} 2$ ).

665 Portz, J. D. V. Colonel. Amsterdam. Cabinet of minerals, fossils, stones etc. sold Amsterdam I8. III. I754 (Boehmer I785 I p. 396; Vosmaer I790).

666 Posthumus, Vincent, son in law of Simon Schijnvoet (Argenville 1742 p. 215-'16: cabinet Rumphius via Schijnvoet and Vincent Posthumus to the town of Amsterdam; but he corrects this in the later edition I780 p. 348 note; Argenville 1757 p. I47; Lesser 1756 p. 80: Posthumus got the cabinet of his father in law Schijnvoet, catalogue I744 Amsterdam). This catalogue of the shells is quoted by Boehmer I785 I p. 394 (Vosmaer I790). A second catalogue is mentioned by Cobres I782 I p. I3I and 
Murray 1904 I p. I82, III p. I67, dealing only with the minerals, fossils and stones.

667 Potr, Joh., Amsterdam. Museum. Valentinus I7I4 II p. I9; Kanold I727 p. 22, 182.

668 Pouderoyen, L. P., Rotterdam. Shells. R.

669 Priester, L. DE. Apeldoorn, earlier master of U.L.O. in Voorburg. Shells especially East Indies. R.

670 Prins, C. H. Overveen, Shells. R.

67 I Quaestius, Joh. Wijbrandus. Cabinet catalogue in 3 pts Leeuwarden 1827 (Engelmann 1846 p. 9).

672 Quina, Dr. Abraham. Cabinet (Uffenbach 1754 III p. 689 v I7Io).

673 RaAt, Henrik van. Rotterdam (near Groote Markt). Druggist. Cabinet of shells (Valentijn I726 III p. 562, I754 p. 46).

674 Rade, von. Merchant of birds. Amsterdam. (Uffenbach I754 III p. 609 V I7IO).

675 Radermacher. 's Gravenhage. Cabinet of Chinese Simplicia, sold i765. (Vosmaer I790).

676 Raije van BreukelerwaArd, Joan. Utrecht and Amsterdam. Cabinet of shells, insects, birds etc. Ireland I790 p. I80 (v I789); Frisch I8I6 p. 35 (v I797); Rudolphi I804 p. I09-III (v I802); Nemnich I809 p. 37 I (v I808); Haupt I8I4 p. 337 (v I8Io); Stoll I788, p. 91, 95, 98, 102, 139, I 56, I $59 ;$ I $788_{2}$ A p. 25,3 I, $35,40,4 I, 55,59,60,63,65,66,74,75$, B p. 8 , 9, I I, I2, I6, I7, I9, 24, 26, 27, C p. 9, I0, I2, I3, I4, I5, I8, 20, 22, 28, 29, 30, 3I, 32, 35, D p. 2, 4, 68; Cramer I779-'82 III p. 4I, IV p. 3I, 44, I4I, I 5I, 166, I7I, I74, I82, 196, 200, 20I, 203, 208, 210, 212, 217, 221, 222, 224, 227, 230, 236, 237, 238, 239, 240, 24I, 242, 243, 244, 245, Appendix p. 132, I52, I57, I58, I60, I63, I67, I69, I75, I79, I8r ; Temminck I808-'o9 I p. IX, 24, 27, IV p. 2I, V p. 38, VI p. 49, 55, VII p. 61, 66; Snelleman on H. Schlegel p. I77, part of collection to Leiden; Murray I904 III p. I22; The cabinet was sold 3. VII. 1827 seq. in Amsterdam in his house Heerengracht 29 between Utrechtschestraat and Reguliersplein (A with prices, E); Stresemann I923 p. I20; G p. I6I.

677 RaU, (Ravius) Prof. Dr. Johannes Jacobus. Raden (Schwäbische Kreits I668 - Leiden I8. IX. I719) Prof. med. Leiden (I7I3). Well known for his operations of the stone. His anatomical collections to University Leiden. Van der Aa 1852-'78 X (b); Index supellectiles anatomicae quam Acad. Bat. quae Leidae est legavit J. J. Rau. Lugd. Bat. I725, ed. B. S. Albinus (in UBA also Murray I904 III p. I2I); Idem, cujus venditio fiet 18. X. I77I (UBA); Suringar I866 2 p. 18-24; Francq I773 p. 38 note; Seba I734-'65 I p. 80 and introduction by Boerhaave; Valentinus 1720 Amphitheatrum zootomicum (D. Joh. Ravii, Collegium ana- 
tomicum), in A; Gedenkboek 1932 p. I8I; Geyskes \& van der Klaauw I934 p. 7; Uffenbach I754 III p. 62I, 625, 640 (v I7I0); Erndtel I7II p. 83 ( $v$ I706-'07); the later travellers mention Rau's collection in the Anatomy: Haller I883 p. 39, 52 (v I725); Grimm I775 p. 305 (v I774); Czermak I879 p. 175 (v I850).

678 Reclaire, Dr. A. Hilversum. Coleoptera, Rhynchota. R.

679 Reenen, Mr. G. C. J. van. Amsterdam Insects. Sepp i762-186o IV p. 83.

680 Rees, P. A. van. Zutphen, 23. XII. I8I7-Putten I9. XII. I902. "Vice-admiraal". For his life see Wereldkroniek 27. XII. I902; Catalogue of shells, La Haye 1900, identified by G. B. Sowerby in $\mathbf{A}$ and in possession of Mrs M. Payne Best-van Rees.

68 I Reformé. Amsterdam, "Kapitein". In a M. S. on insects (Elliger?) in A. 682 Regnard, Maastricht. Consul. Cabinet of fossils. Sander 1783 I p. 608 (v 1777 ).

683 Reigersbosch, Jan. Insects. Sepp. i762-i860 I, 6 p. I9.

684 Reyners, General. Insects. Sanders i783 p. 582 (v I777).

685 Reynst, see Reinst.

686 Reiner, D. DE 's Gravenhage. Anatomical collection, bought by the King. Rudolphi I804 I p. I39-I40 (v I802); Horn I83I p. 345 (v I830).

687 Reinkingh, W., 5. I. I82I-IV. 1905. Schoolteacher. Shells to Zoological Gardens Amsterdam. (Catal. in M. S. in A).

688 Reinst, Georg = Gerrit Reynst. † 29. VI. 1658. "Lid vroedschap". Amsterdam (Keizersgracht, E. side, north of Hartenstraat), son of the Gouverneur-Generaal. Fuchs I9I6 p. 6, 39 (v I663); Valentinus I7I4 II p. I9; Kanold 1727 p. 182; Jensen 1919 p. 45.

689 Reinwardt, Prof. Dr. Caspar Georg Carl. Lüttringhausen 5. VI. I773Leiden 8. III. 1854; Prof. med. Harderwijk (180I); Director of gardens, menageries and cabinets of King Lodewijk Napoleon (Soestdijk, then Haarlem, then Amsterdam); Prof. Chem. Pharm. et Hist. Nat. Amsterdam (1810). In 18I5 he went to the East Indies as Director of Agriculture where he collected much, but the collections were shipwrecked during the transport to Europe. In 1823 Prof. chem., bot., geol., mineral. Leiden. Gedenkboek I932 p. 56, 25I, 275, 66I (b); Van der Aa I852-'78 X (b) ; Collot d'Escury I844 VII p. 286, 339; Veth, P. J. Zevental Levensschetsen I884 p. 95 ; Hoffmann I868 II p. 340 (v I836) ; Horn \& Kahle I935-'37 p. 222; Molhuijsen I9I I-'37 IV Kol. I I35 (b) ; G p. 31, 87.

690 Renard, P., Amsterdam. Cabinet of minerals etc. sold I782 (Vosmaer I790).

691 Rengers, Hans Willem Baron. 's Gravenhage "Lieutenant Général de la Cavallerie, Président du Conseil de la Guerre, Chambellan de Mme la Princesse d'Orange". Cabinet. Argenville I780 p. 346; Voet I766 I p. 27, $35,45,59,67,74$, IO0, IOI, I02, I07, II p. 19, 21, 22, 28, 46, 47, 69 Stoll I $788_{1}$ p. 20, 21, 22, 23, 24, 25, 3r, 58, 6I ; I788 2 p. $28,30,33,35,36$, 
37, 4I, 44, 48, 49, 75; Cramer I799-'82 I p. I 5 Intr., I9, 26, 40, 48, 54, 55, $61,67,79,85,88,92,93,97,104$, I I4, I I9, I22, I24, I25, 128, I29, I33, I40, I4I, I44, II p. 8, 12, I4, I8, 28, 29, 39, 43, 45, 47, 52, 53, 58, 59, 61, 62, 76, $78,79,84,95$, IOI, I02, I 10, I28, III p. 2, 4, 10, 22, 26, 42, 48, 63, 64, 65, $76,78,96,98$, I21, 123, I24, I37, 140, I57, 165, I66, I72, IV p. 44, 52, 64, $65,67,69,70,73,83,95,96$, I I 5, I 16, I 19, I24, I26, I4I, I42, I 54, I58, 175, I96, 210, 24I, 242, 245; Pallas I767-'77, VIII p. 45 IX p. 24, 27.

692 Renner. Amsterdam. Cabinet. Valentijn I726 III p. 56I; I754 p. 45 ; Kalff I928 p. 207.

693 Rensselaar, Anthoni van, Willemszoon. Amsterdam, lived on his estate Craailo near Naarden. Cabinet of shells, insects, birds. Cramer I779-'82 II p. IO, IV p. 207, 232; Nozeman I770 I; Argenville I780 p. 355 ; Sander I783. I p. 576 (v I776), spelt wrongly Reuszelaer.

694 Rensselaar, Johan Jacob van. Amsterdam. Probably brother of preceding with whom he had together the cabinet (Nozeman I770 I); Sepp I782I860 I poems in preface. See literature under preceding.

695 Rentre, zie Roetert.

696 Resner (Resnerus). 's Gravenhage (gentilhomme de Zéelande). Cabinet. Kanold 1772 p. I98; Misson 1722 I p. 14 (v I687) ; Uffenbach I754 p. $3^{88}$ (visited I7IO Miss Bayet, "eine Verwandtin und gewesene Haushalterin von Herrn Resnerus").

697 Rethaan Macaré, née Ontijd, Mrs. Florentine Jacobine Martina, I8I2Utrecht 30. IX. I887. Cabinet of shells begun 1824. Catalogue of sale 6. VIII. I888 Utrecht (A parts to Schepman, Zool. Gardens Amsterdam etc.); G p. I69.

698 Reuve, DE, Delft. Cabinet. Gersaint I736 p. 20.

699. Reverhorst, M. C. van, Leiden. Burgomaster. Cabinet sold 16-20. VI. I8I7 Leiden (Lugt I938 p. 9165).

700 Revier, Le. Delft. Museum. Valentinus I7I4 II p. I9; Kanold I727 p. 195 (m); Uffenbach I754 III p. 339 (v r7ro).

7OI Riemer, P. DE. Rotterdam. Cabinet of Anatomy. Catalogue I83I in UBA.

702 RiemsdijK, Jonkheer Adriaan Willem Gerrit van. I803 castle HelmondUtrecht I87r. "Lid van het Munt College, Inspecteur-Essayeur-Generaal." Cabinet of shells to his son (follows).

703 RiemsdiJK, Jonkheer B. W. F. van. Former Director Rijksmuseum (Art) Amsterdam. He gave the collection of shells of his father (preceding) in I92I to the Zoological Museum Amsterdam.

704 Rijk, J. C. Major. Meersen. Butterflies. Lempke 1936 p. 239. R.

705 Rijkevorsel, Mr. Dr. Corneille van, Cabinet of nat. hist. sold 19-3I.X. I778 Amsterdam (Lugt $1938 \mathrm{n}^{\circ}$ 2899).

706 Ritsema, Coenraad, Cz. I846-i929. Conservator entomology RijksMuseum Nat. Hist. Leiden. He was 1876-'80 conservator of Entomol. Soc. The collections were given I880. to the school of Agriculture in Wageningen. Blasius I880 p. 19 (v I879); Horn \& Kahle I935-'37 p. 226; G p. 273. 
707. Roding, G. M. Amsterdam. Shells. R.

708 RoedaAg, A. Leiden. Cabinet of anatomy, sold I753 (Vosmaer I790).

709 Roede, DE. Dealer in objects made of naturalia (Uffenbach I754 III p. 595, V I7IO).

710 Roeters (Roetert), Dr Ernst. 1616-85. Amsterdam. In de vergulde Ketel, Singel between Warmoesgracht and Hartenstraat. "Schepen, Vroedschap". His cabinet was well-known. Boccone I674 p. 278 (v I673); Monconys I666 II p. I69 (v I663 s. n. Rentre Heent); Hoogewerff r9i9 p. 68, 69, 70 (v I667); Kanold I727 p. 182 ; Elias Vroedschap van Amsterdam I p. 488; Fuchs I9I6 p. 5, I9 (v I663); Valentijn I726 III p. 56I (m); I754 p. 45; Murray I904 II p. 232 mentions a catalogue of sale Amsterdam I685. In the Amsterdam Archives Mr. Voorthuyzen was so kind to call my attention to the inventary made by notary G. Steeman after his death dated 7. III. I685 (Not. Arch. 2642); Vosmaer I790 mentions: Ernst, Dr. Roetter, Amst. I685, div. nat. and curiosities; Jaarb. Amstelodamum XIV I9I6 p. 205, 206.

7 I I Roeters, Mr. Jan. 1614-27. XII. I667, brother of preceding, lawyer, Amsterdam, "commissaris voor de kleine zaken, secretaris van Amsterdam". (Elias, de Vroedschap van Amsterdam I p. I84, 367, 368, 494, II 653; Jaarb. Amstelodamum XIV I916 p. 207, 253 seq.); Fuchs 1916 p. 7.53 (v I663); Hoogewerff I9I9 p. 60 seq. (v I667).

712 Roeters van Lennep, Mr. Herman Christiaan. Amsterdam 20. XII. I820Twello ("de Parkeler") I 5. VIII. I879. Merchant Amsterdam till I862, later Twello. Cabinet of shells sold I8. VII. I876 seq., catalogue in A, also M. S. Catalogue presented in 1885 to the Zoological Gardens Amsterdam by the heirs. The Society had bought many shells at the auction.

713 Romijn, Dr. Gijsbert. Apeldoorn Io. I. I868-Haarlem 20. I. I930. "Inspecteur van de Gezondheidsdienst." Made large collections of Dutch aquatic animals.

7 I4 Roo, W. DE, Cabinet of birds etc. sold 's Gravenhage I760 (Vosmaer I790)

7 I5 Rooy, A. L. DE, litt. human. stud. Harderwijk in I8IO, collected insects (Sepp I762-I860 IV p. 92).

716 Rooy, see Medenbach de Rooy.

717 Roon, Gerrit van. Rotterdam I870-I928 Coleoptera. Everts 1922 p. XII; Horn \& Kahle I935-'37 p. 230; M. S. in E.

718 Roozendaal. Here the Dukes of Gelderland had a menagerie (Witkamp I872 p. V; Loisel 1912 I p. I 56 seq.).

719 Ros, Joannes. Amsterdam. The only man in Amsterdam who knew how to clean the shells for cabinets perfectly (Valentijn I726 III p. 56r ; I754 P. 45).

720 Rosendael, J. B. J. van. Merchant Dordrecht, $\dagger$ Amsterdam I6. III. I884. Collection of shells bought 1859 by Zoological Gardens Amsterdam.

721 Rossum, van. A Gorgonarian in Zoological Museum Amsterdam comes from collection van Rossum. 
722 RotTERDAm. Here again the fair is the place where many wild animals were shown (Brown 1682 p. 4, v I668, lions, leopards; Witkamp I872 p. VIII repeats this; Evelyn I879 p. 27 v I64I : elephant; Dethmar I838-4I p. I27). The last mentions a piece of wood with shipworms in the "Beurs" (Exchange).

The anatomy-hall of course contained anatomical collections (see: de Bils, Aan alle ware liefhebbers der anatomie, Rotterdam 1659 in UBA, inciting to the foundation of an anat. cabinet) and other things (hair of Mary Stuart, Haupt I8I4 p. 282) ; Uffenbach I754 p. 309 (v I7Io); Honoré I779 p. 28 (v I776); Björnstâhl I782 p. 363 (v I775); Schaeffer I794 p. I08 (v I788); Haupt I8I4 p. 282 (v I8Io); Meiszner I8I9 p. 83 (v I8I7 "comical anatom-theatre"); Horn I831 p. 344 (v I830); Mac Gregor I835 p. I4I, 264 ( $v$ I833). In 1740 a catalogue was issued (UBA), mentioned by Vosmaer under A. Later on Rotterdam had a complete medical school like other towns (Terwen I855 p. 268).

The druggist-guild also possessed all sorts of natural curiosities (Cohen, in: Pharmac. Weekbl. $\mathrm{n}^{\circ} 5$ I930 p. 94).

Of course we must mention the "Bataafsch Genootschap voor de Proefondervindelijke Wijsbegeerte", founded I77 I by Steven Hoogendijk. It issued "Verhandelingen" since I774. (Vrolik in Versl. K. Akad. Wet. Amsterdam V p. 36). It is mentioned by Honoré I779 p. 286 (v I776); Sander I783 p. 469, 477 (v II77); Björnståhl I782 V p. 360 (v I 775); Haupt I8I4 p. 286 (v I8Io); Meiszner I8I9 p. 9 (v I8I ) ; Fliedner I83 I p. 393 (v I823-'24); Mac Gregor I835 p. I38 (v I833); Dethmar I838-'4I p. IO9 (v I835); Beets I932-'33 p. 80; Nyegaard 1929 p. I46 (v I836). It seems however that this Society was not specially interested in natural curiosities. The Zoo: "Rotterdamsche Diergaarde" was begun in 1855 . Jaarbericht I868-'69, tevens bevattende de korte Geschiedenis, p. I63; Jaarbericht II I869_'7I ; also in Der Zoolog. Garten VI p. 65, VIII p. 20I, IX p. 307, XI p. 381, XII p. I59, XIII p. 335, 280, XXX p. 182, 250, XXXIII p. 284, XXXIX p. 352, XL p. 257, XLI p. 355, XLIV p. I84, XLVIII p. 33, LVI p. I36, LVII p. 129, LIX p. 50; Witkamp I872 p. XXIV; Loisel I907 p. 85 ; I9I2 III p. 212 ; Terwen I855 p. 268-'69; Flower, Special Rept n² 2, Cairo Nat. Print. Dept. I908; De Rotterd. Dierg., Rotterdam I858 (UBA A); Blasius I880 p. 25 (v 1879); Musea 1938 p. 253 ( $\mathrm{n}^{\circ}$ 194); G p. 177.

Since 1927 Rotterdam possesses a beautiful Natuurhistorisch Museum "Wethouder Hoffmann" (Musea 1938 p. 249, n 190 ; Lempke 1936 p. 245). The cabinet of the Erasmiaansch Gymnasium is worth mentioning.

723 Rousset. 's Gravenhage. Worms and shipworms. Jordan i736 p. I88 (v I733). 724 Roy Dr. Cornelius Henricus À, Physician Amsterdam. † I4. VII. I833. Van der $1852-' 78 \mathrm{X}$ (b); His library (Catalogue 1830 in A) was extremely rich. He had a cabinet: Stoll 1813 A p. 74, B p. 28, E p. 2, 3, 4; Nemnich I809 p. 370 ( $\mathbf{v} 1808)$. 
725 Roy, J. P. À. Amsterdam. Cabinet. Sepp. 1762-1860 III p. 67.

726 RozendaAl, see Roosendaal.

727 Ruysch, Prof. Dr. Frederik. 's Gravenhage 28. III. 1638-Amsterdam 22. II. I731. Physician in the Hague, then Amsterdam (I667 praelector, I668 professor anat. of the surgeon's guild, I672 "stadsvroêvaêr", I685 prof. bot. Athen. Amstel.). Molhuysen I9I I-'37, III Kol. I 108 (b) ; Van der Aa I852-'78 X (b) ; Scheltema, Het leven van F. R., dissert. Leiden I886 (b) ; Schreiber, Hist. vit. et met. F. R., Amsterdam 1723 (b) ; Gedenkboek I932 p. 37, I80 seq., 265, 668 (b) ; Collot I844 VII p. I93-'94, 320-'2I (b) Maitland I855 p. I6I; Nuijens I928 p. 30 ; Album Amicorum F. Ruysch in UBA. He possessed a large anatomical and zoological cabinet, published in; Mus. anat. R. I69I ; Thesaurus anatomicus I-X, I707-'26 (more editions of each), Curae post. seu Thes. max. I724; Thesaurus animalium I710 (1725) etc. (on his works see Molhuijsen 1.c.). His first collection was sold 1717 for $f 30.000$ to Czar Peter of Russia (Scheltema, Peter de Groote I842 I p. II8-I2I, II p. II,79, Beckmann, I777, Physic. Ökon. Bibl. VIII p. 493 (Libr. Techn. Hoogeschool Delft); (Raptschinsky 1936 p. I23) and the second (Catalogue I73I, UBA) to the King of Poland after his death for $f 20.000$. His collections are mentioned in: Rumphius I705 p. 3I8; Valentinus I7I4 II \& III App. XIIX (= I8) p. 59; Valentijn I726 III p. 56I, I754 p. 45 ; Lesser I744 p. $34,43,63 ;$ I756 p. 82-83; Kanold I727 p. 22, I82; Argenville 1742 p. 215; I757 p. I47; I780 p. 342 ; Wagenaar, Amsterdam in zijn opkomst etc. I767 III p. 240; Seba 1734-'65; Museum Petiverianum I695-I703 p. 46, 95 (A); Czermak I879 p. I74I75; Smith I796 I p. II ; Honoré I779 p. 64; Hoogewerff I9I9 p. 79, 80, XVIII (v I667-'69); Boccone I674 p. 278 (v I673-'74); Brown I682 p. 23 (v I668); Erndtel I706-'7 p. 83 (v I706-'7); Uffenbach I754 III p. 639 (v I7IO) ; Haller I883 p. IOI, 5I, 54, (v I725-'26); Murray I904 I p. II6-II7, III p. I47; Cole I9I4 p. 33; van der Klaauw 1926 p. 4 ; Geijskes \& van der Klaauw I934; Horn \& Kahle I935-'37 p. 234.

728 Ruysch, Dr. Hendrik, Amsterdam $\pm 167 \mathrm{I}$, son of preceding. Physician. Amsterdam. Schreiber, Vita Henr. Ruyschii (b) ; Gedenkboek 1932 p. 669 (b) ; He helped his father and edited 1718 in Amsterdam a new edition of Jonston's Theatrum animale. His Album Amicorum in UBA; Haller I883 p. 52, IOI (v I725-'26).

729 Ruloffs, Bartholomeus, Amsterdam. “Organiste, Chef d'orchestre”. Cabinet of shells etc. sold 22. XII. I80I (Lugt $1938 \mathrm{n}^{\circ} 6344$ ).

730 Rulters, Amsterdam. Museum. Valentinus I7I4 II p. I9.

731 Rumphius, Georg Eduard, 1634-15. VI. I702. Merchant for the East Indian $\mathrm{Cy}$ in Amboina. Rumphius Gedenkboek, Haarlem 1902 (b etc.) ; Collot I844 VII p. 307-8, 310, 314-7; Greshoff in: Encycl. Ned. Indië 1902 p. 464-8 (b); Greshoff in: Guide Expo. Marseille I906 p. 35-40; Maitland I855 p. I68; Murray I904 I p. I47, III p. I46; Henschel, Clavis 
Rumphiana, acc. vita Rumphii 1833 (A); Harting, in: Album der Natuur I885 p. I ; Sirks, M. J. I9I5, Indisch Natuuronderzoek p. 25; Veth, H. J. Dissertation 1879 Leiden, p. 5-8; Kalff in: Indische Verlofganger 1930 -31 p. 45-6; id. 1928, p. 206, 208; Bloys van Treslong Prins in: Tijdschr. Bat. Gen. LXIX, 1929, p. 426-35 (!) ; Rumphius is known to the whole wolld for his Amboinsche Rariteitkamer 1705 and later editions, wherein he figured his zoological collections. According to Argenville 1742 his collections came via Schijnvoet and Vincent Posthumus to the town of Amsterdam but $I 780$ he corrects this. It was Schijnvoet who took care that the book was published as is clear from the text. It is certain that Rumphius sold shells in 1682 to Cosimo III de Medici of Toscane. Le collezioni di Giorgio Ev. Rumpf, acquistate dal Granduca Cosimo III de Medici una volta esistenti nel Museo di fisica e storia naturale di Fírenze, Firenze 1903; Greshoff in Album der Natuur 1909: Nic. Witsen als Maecenas: "on Witsen's instigation R. sent shells to Cosimo"; Godée Molsbergen in Med. Hist. Inst. Roma (2) I, I93I, p. 163-7 mentions shells after Rumphius on marble inlaid tables in the Palazzo Pitti in Florence; Kalff I928 1.c.; Horn \& Kahle I935-'37 p. 234: "Sammlg. weder in Mus. Stor. Nat. Firenze, weder in Mus. Civ. Stor. Nat. Genova”; Pulle however in Encycl. Ned. Indië, 2d ed., I9I9 p. 640-5 points to the Correspondence on the shells, which now are in Staatsmuseum Wien; Chemnitz I760 p. I 30 says that the Cabinet of Rumphius was in the possession of von Gärtner in Wien. See further: Valentijn I726 III p. 560; I754 p. I, 43; Argenville I742 p. 26, 27, II4, II5, 215; I757 p. 6, I47; 1780 p. I8-I9, 348-9; Lesser I744 p. 33, 43; I756 p. 35, 48; Uffenbach 1754 III p. 675; Molhuijsen I9I I-'37 III Kol. I IO4 (b).

732 Rurynes, de (or de Ruvynes). Maastricht. "Colonel d'Infanterie". Cabinet corals, minerals, fossils (Argenville $\mathrm{I} 78 \circ \mathrm{p}$. 8I I).

733 Rüschкамp S. J., F., earlier Valkenburg, now Maastricht. Coleoptera. Everts 1922 p. XII. R.

734 Rutger, Johan: Amsterdam. Shells (Lesser I756 p. 82).

735 Ruvynes, see Rurynes.

736 Salp. Offered curious animals to King of France (to get a coat of arms) - (Regnard I68I).

737 Sandifort, Eduard, Dordrecht I4. XI. I742- Leiden 12. II. I814. Prof. anat. Leiden. Described the Anatom. Museum of the University I792. He had also a private anatomical collection which 1802 was offered for sale on the Anat. Theatre (Rudolphi I804 p. 132). Sander I783 I p. 527 (v I776); Grimm I775 p. 305 (v I774); Aikin 1920 p. 220 (v I784); Schaeffer I794 p. 122 (v I788); Smith I796 p. Io (v I786); Anonymus I792 p. 87 (v I790).

$73^{8}$ Sandifort, Prof. Dr. Gerard, Son of preceding. Leiden 3I. I. I779- II. V. I848. Prof. anat. et phys. Leiden. Van der Aa I852-'78 X (b); Van der 
Klaauw 1926 p. 126 a.o.; Geyskes \& van der Klaauw 1934 p. 13; Maitland I855 p. I67; Rudolphi I804 p. I3I (v I802); Meiszner I8I9 p. 76, 77 (v I8I7); Otto I825 p. 456 (v I822).

739 Sarraz, see Lassara.

740 SAS, see Ypelaar.

74I Scaliger, Joseph Justus, Agen 5. VIII. I540--Leiden 2r.I.1609. Prof. Leiden. According to Murray I904 I p. 25 he had a collection of nat. history (he refers to it as: "In nostris musarum thesauris" in: De Subtilitate, Exercitatio I12, p. 422, Francof. I612). Collot 1844 VII p. I83. Van der Aa $1852-' 78 \mathrm{X}$ (b).

742 Schaghen, see Genege.

743 Schaid, Balthazar, born Strassburg, druggist in Amsterdam. Cabinet of shells. Valentijn I726 III p. 56r, 568 (d) ; I754 p. 45, 52-66; Seba I734 - I765 Il p. 9I ; Uffenbach I754 III p. 683 (v I7Io Scheid); Pekarskij 1862; Kalff 1928 p. 207 (speaks of Balthasar Schmidt, which is wrong).

744 Scheepmaker, G., Wzoon. Amsterdam "Instituteur". Cabinet of shells, of which he gave to Zoological Gardens Amsterdam in 1844, see also Kort Verslag Wetensch. Werkz. Commissie Bijdr. t. d. Dierk. p. Io, I5 (A). Catalogue of Sale 24-26. I. I855. Amsterdam (B $\mathbf{n}^{\circ}$ I752). Part of it came to Voorhoeve.

745 Scheid, see Schaid.

746 Schelling, Ir. A. H. Groningen. Shells. R.

747 Schelling, Ir. H. G. J., Architect. Utrecht. Shells. R (bought 1937 coll. De Vos tot Nederveen Cappel).

748 Schep, see Sepp.

749 Schepens, Johannes, Amsterdam. Silversmith. He was an entomologist, in A ( ${ }^{\circ} 3537$ A) a M. S. treatise on the honey-bee. He made microscopical preparations especially of the honeybee and the bean (Nemnich I809 p. $372,373, v 1808$, when Schepens had done this about 40 years).

750 Schepman, Mattheus Marinus. 1847 - Bosch en Duin 19. XI. 1919. "Rentmeester van Rhoon en Pendrecht, Albrandswaard en Piershil" I872-I909, one of the founders of the Nederlandsche Dierkundige Vereeniging. Cabinet of shells now in Zoological Museum Amsterdam, bought 1920 (Sale Catalogue 9-Io. VI. 1920 in A). Verslag Rijks Mus. Nat. Hist. Leiden I9I9I920 p. 6; Journal de Conchyliologie 64 p. 345 (b).

751 Schepman, W. A. Ivangh, Rhoon. † 7. IV. 1878. Insects. Everts 1922 p. XII.

752 Scheveningen. Here was the cheek of a whale in the church. Ferrner igio p. 342 (v 1759); Hoogewerff I9I9 p. 109 (v 1667-'69).

753 Schijnvoet, Simon. 's Gravenhage I652-Amsterdam 24. VIII. I727. "Onderschout, Hoofdprevoost, Geheimschrijver Aalmoezeniersweeshuis”. He lived Kerkstraat between Spiegel- and Vijselstr., in "'t Spingend Fonteyn" He was a good engraver and garden-architect and possessed rich collections of antiquities, coins and natural history. He edited the Rariteit- 
kamer of Rumphius I705. Collot I844 VII p. 200; Two letters in UBA; Van der Aa I852-'78 X (b); Valentijn I726 III p. 56I, 563 (d); I754 p. I5, 26, 28, 45, 48, 50, 5I, 52, 58, 62, 63, 64, 65, 66, 67, 68, 70; Argenville I742 p. 215-6 says that he possessed the Collection Rumphius, but later corrects this; I757 p. I47 and I780 p. I8, 348-49; Gersaint I736 p. 40 made the same mistake; Uffenbach I754 III p. 668 (v I710); Blankaart, Schouburg I688, preface, p. II, 44; Pekarskij I862 (Schijnvoet's museum was visited by Czar Peter, and Schijnvoet had many connections with Russian resident Christoffel van Brants); Amstelodamum Jaarb. XXVI 1929 p. I26, I37. His cabinet passed to his son in law Vincent Posthumus, who 1744 offered it for sale in two catalogues, mentioning that it originally came from his father in law Schijnvoet. The catalogue of shells is quoted by Boehmer I785 I p. 394 and Vosmaer 1790, that on fossils, stones and minerals by Cobres I782 I p. I3r. See also Murray I904 I p. 182, III p. I67, Lesser I744 p. $34 ;$ i756 p. 36,80 ; Lugt $193^{8} \mathrm{n}^{\circ} 377$. In $\mathbf{B} \mathrm{n}^{\circ}$ I0 4 the sale catalogue of Schijnvoet's books 2. II. I728, no nat. hist.

754 Schlosser, Dr. Joan Albert, † i768 or '69 Physician. Amsterdam (i755). He had a large cabinet, from which he began to describe (1768) the Lacerta Amboinense. After his death it seems (Sander I783 I p. 594, v 22. VIII. 1777) that Boddaert got the cabinet. He actually continued the description: De Chaetodonte Argo I770, De Testudine Cartilaginea I770, De Rana bicolore I772, De Chaetodonte Diacantho I772. Vosmaer I790 mentions a sale catalogue Amsterdam i769. See also Ferrner i910 p. 356 (v I759); Anonymus I767 p. 327 ; Pallas I766 1 p. 266 (Boddaert 1768, p. 33I) ; $1766_{2}$ p. 60 (Boddaert I770 III p. 7, 24, 25, V p. 26); 1767'77 VIII p. 39, X p. 31 ; Houttuijn I76I-73, I7, p. 399; Navorscher 58, p. 293.

755 Schmidt, see Chatelain.

756 Schmitz S. J., H., earlier Sittard, now Bonn. Insects: Diptera. Everts 1922 p. XII. R.

757 Scholte, (cf. Scholts) Valentijn 1726 III p. 562, I754 p. 46.

$75^{8}$ Scholten, I. A., Cabinet sold Amsterdam 1773 (animals in liquor) (Vosmaer I790).

759 Scholten, L. H., Lobith, Lepidoptera. Lempke 1936 p. 239.

760 Scholts, Dr., Rotterdam. Rumphius i705 p. 337 (cf. Scholte).

761 Schoonhoven, widow. Cabinet of divers naturalia sold Amsterdam 1775 (Vosmaer 1790).

762 Schorer, Daniel Steven. "Oud burgemeester, schepen en raad van Middelburg, Bewindhebber Oost. Ind. Cie. ter kamer Zeeland." Cabinet of shells, birds, etc. sold 9. V. I79I at his house: Vlissingsche straat, Middelburg. (B $n^{\circ} 572$ p. 57 ).

763 Sснот, Jacob Elias, Amsterdam. Cabinet of Shells. Valentijn 1726 III p. 56I, 566 (d) ; 1754 p. I9, 45, 50, 52, 56, 62, 63, 64, 65, 66, 67; Uffenbach I754 III p. 690 ; Kalff I928 p. 207. 
764 Schot. "President de la Cour de Brabant" Lyonet bought his cabinet (Hublard I9io p. 87).

765 Schouwman, Aart, Dordrecht 4. III. I710-'s Gravenhage 5. VII. 1792. Painter. Van der Aa 1852-'78 X (b); Van Gool 1750-'51 II p. 346 (b portrait 212); he made many drawings for Vosmaer I766-I804 (some originals in A) and Pallas; Boddaert I770 I p. VIII.

766 Schrammerdam, see Swammerdam.

767 Schravenwand, Utrecht. Cabinet of shells. Anonymus i767 p. 328, Argenville $\mathrm{I} 780$ p. 8I I.

768 Schrieck, Otto Marcelis van. Painter. (After Immerzeel II p. I99 says Jensen 1919 p. 49 note this man is meant by Monconys I666 II p. I6I, when he writes that he visited 20 . VII. I663 "à Ouater reik Otho et ses tableaux") Monconys 1.c. saw his butterflies on a place called Waterrijk.

769 Schröder, Samuel. German druggist in the Hague (Seba I734-'65 II p. I05).

770 Schubaert, Toers Diesbergen. Harderwijk I8. II. I805-Utrecht 4. X. I854. Prosector Anat. at University and Veterinary School Utrecht. Had a cabinet of anatomy, collected also shells and insects. Catalogue of sale I. VII. I856, Utrecht (in A) ; M. C. Verloren, J-evensschets van T. C. Schubaert, $1854, \mathbf{b}$ (in $\mathbf{A}$ ), also in preface of Catalogue; part of his collections came in the possession of Eyndhoven (see Eyndhoven I86r). Horn \& Kahle 1935-'37 p. 250.

771 Schuylenburgh, Burgomaster of Haarlem, probably Mr. Coenraad, 's Gravenhage Ir. VII. I7I4-Haarlem 21. XI. 1754 (see H. H. Roëll in Ned. Leeuw I906). Gersaint 1736 p. 20 ; Lesser 1756 p. 83.

772 Schuyт, P. J. M. 1870-1927. Leeuwen (Gelderland). Lepidoptera. Lempke I936 p. 239, Everts 1922 p. XII ; Horn \& Kahle I935-'37 p. 252 (Dutch Lepidopt. now in Zool. Mus. Amsterdam).

773 Schultz, Dr. H., Physician. Utrecht. Insects. Cramer I779-'82 I p. IOO, -. II p. II, 22.

774 Schutt, see Schutte.

775 Schutte, Dr. J. G., Physician, Utrecht. Cabinet. Argenville I780 p. 337 (especially shells and insects); Boddaert 1768 p. XXIII (J. G. Schutt Med. Dr.) ; Cramer I779-'82 I p. 98, 120, II p. 24, 31, 33, 43 (G. I. Schutt, med. Dr. Utrecht). His cabinet was sold Utrecht I778 (Vosmaer I790).

776 Schuuttrup, A. C. Amsterdam. Insects. Cramer I779-'82. Appendix p. I49.

777 Schwammerdam, see Swammerdam.

778 Schwenke, see Schwenken.

779 Schwenken, Martinus Wilhelmus. I707-'85. Med. Dr. 's Gravenhage. Grimm I775 p. 289-'90 ( 1774 cabinet divers animals and a garden); Forster I843 p. 90 ( $v$ I778); Ehrhart I783 p. 238 (v I782 "liest in seinem Garten den hiesigen Apothekern ein Collegium"). Murray I904 mentions an Index Musaei Schwenkeniani, 1785 by Meuschen (also Vosmaer 1790 who mentions the sale of the cabinet 1785 ). His library was sold 26 . IX. I785 
(B 490) but only contains p. I39 a microscope of Leeuwenhoek. Baster (Natuurk. Uitsp. 1765 II 2 p. $5^{8}$ ) got a fresh water Medusa from Schwenke.

780 Seba, Albertus. 2. V. I665. Etzel-Amsterdam 2. V. 1736. Apothecary in Amsterdam. Author of the well-known Thesaurus (Seba 1734-'65), in which he described his cabinets. The first he sold 1717 to Czar Peter of Russia, the second was sold long after his death on I4. IV. I752 seq. in Amsterdam. In Svenska Linné-Sällskapets Årsskrift, XX, I937 p. 75IOO I have collected all I know about him, so may refer to that paper and add here some omissions : Kort verslag Wetensch. Werkz.heden verr. d. L.eden v. d. Commissie v. Bijdr. t. d. Dierk., Verg. 21. XI. I852, in A [Roeters van Lennep shows a shell of Seba III t. 59 f I, 2, Delphinula arion, see also Catalogus Roeters van Lennep p. $33 n^{\circ} 621$ ] ; Catalogue Leers... 2nd title, after Preface; Francq van Berkheij, Catalogue of his books, sold I783: I p. I04, I09, I24-25, I49, I 59 from which it follows that he assisted Vosmaer in the edition of vol. III and IV of the Thesaurus; In the FamilyArchives Vosmaer, where I was kindly admitted there are many important letters on the edition of the Thesaurus: Pallas and Houttuijn assisted Vosmaer, Houttuijn made the Dutch Index, the French translation was made by Dr. Ducloux under the supervision of Allamand, after Massuet in Amsterdam had begun the work. It follows from these Archives without doubt that Vosmaer edited about half the third and the whole fourth part of the Thesaurus; Pallas I766 p. 219 (Boddaert I768 p. 274); Argenville 1742 p. 216; I757 p. 147; I780 p. 349; Lesser I744 p. 55-56, I756 p. 73-75, a poem he had destined for Seba's III vol., but which reached him after his death, so that it was not used; Valentijn I754 p. 27, $45,48,49,5 \mathrm{r}, 52,63,64,65,66,67$; Houttuijn I76I-'73, 4 p. 326; Björnståhl I782 V p. 4I6 (v I775); Sander I783 I p. 522 (v I776); Dict. Sc. Nat. 6I p. 219; Gedenkboek I932 p. 25I; Vosmaer I790 cabinet sold I752; Horn \& Kahle I935-'37 p. 253 ; History Coll. Br. Mus. Nat. Hist. II 1906 p. 5,43 (s.v. Lidth de Jeude).

781 Segveld, see Segveldt.

782 Segveldt, Johan van. 's Gravenhage. "Bewindhebber der W. Ind. Cie". "Offiecier de la Cour". Cabinet of shells. Gersaint 1736 p. 40; Valentijn I726 III p. 562, 57 I (d) ; I754 p. 27, 46, 55, 63, 64, 66; Hublard I9ro p. 89; Argenville 1742 p. 215 ; I757 p. 147; I780 p. 342. Murray I904 III p. I69 mentions a sale catalogue I739. [N.B. In I739 a miss Segvelt married a Mr Oldenbarnevelt, her daughter married Mr de Loches, see there].

783 Segvelt, see Segveldt.

784 Segwaard, Bartholomeus van. Dordrecht. "Oud-Raad, Schepen, Ontvanger der verponding”. Cabinet of shells. Valentijn I726 III p. 562, 573 (d) ; I754 p. $5,7,8$, I I, I2, I3, I5, I9, 25, 26, 27, 29, 46, 47, 49, 52, 54, 55, 56, 63, 64, 65,$66 ; 67,68,69,70$. 
785 Sellius, Prof. Seba got a shell from him for reproduction (Seba $1734-65$ III p. I52).

786 Semmelink, J., 8. VI. I837-i9i2. Medical Officer in East India ( $1859-282$ ). Physician in the Hague. Shells given to Museums Groningen, Amsterdam etc.

787 Sepp, Christiaan. Born Goslar-Amsterdam begin VIII. I775. Amsterdam. Etcher of maps and insects. The last he collected in Sepp i762 $\rightarrow$ (30 first plates are of his hand). Lugt $1938 \mathrm{n}^{\circ} 2460$ mentions the sale of his cabinet 5. XII. 1775 seq. Amsterdam. Van der Aa I852-'78 X (b). Molhuijsen I9I I-'37 V Kol. 719 (b).

788 SePP, Jan Christiaan, eldest son of preceding. Amsterdam 8. XI. I73929. XI. I8I I. Followed his father in work and study. Bookseller. Van der Aa I852-'78 X (b); Molhuysen 191 I-'37 V Kol. 725 (b); Collot 1844 VII p. 338, 209; Maitland I855 p. ı68; Björnståhl I782 V p. 468 (v I774); Nemnich I809 p. 370 (v I808); Tischbein I86I p. I20 (v I772: "Schep", Tischbein made the first plates for: Nozeman I770); Stoll I788 1 p. IOI; Argenville I780 p. 356; Francq I769-I8I I III I p. 22.

789 Sepp, Jan, 1778-i9. XII. I853 Amsterdam. Bookseller. Continued the work of his father (preceding) and grandfather. Entomologist. Molhuijsen I9I I-'37 V Kol. 724 (b).

790 SePP, Cornelis 20. IX. I81o Amsterdam-3I. I. I868. Only bookseller, continued the work on insects with the help of Snellen van Vollenhoven. Horn \& Kahle 1935-'37 p. 255 tell us that no insects are left in the cabinet yet preserved in the family-archives. Molhuijsen I9II-'37 III Kol. I 68 (b).

791 Serik̀re, Ds. Guillaume DE. Naarden 3. I. I788-'s Gravenhage I7. IX. I868. Clergyman Zutphen ( I8I I), Deventer, E. India. Later historian, Governor of the "Moluksche eilanden" (I84I-'45). Kalff in Kol. Tijdschr. IV I9I5, p. 47-72. Shells to Zool. Gardens Amsterdam (I9. IV. 1847).

792 Siemens, Miss T. J. Haren (Gron.) Shells. R.

793 Siккемa, H., Wageningen. Insects. Lempke i936 p. 239. R.

794 Sillem, J. A., Bussum. Collection of palaeartic birds together with van Marle (bought from Snouckaert). $\mathbf{R}$.

795 Sipman, Dr. Johannes Philippus, Physician once with Rumphius in Amboina. Returned with beautiful cabinet to Holland, went again to India and gifted many shells to the "Opperlandvoogd" Joh. van Hoorn and others. See Rumphius I705 p. I67; Valentijn I726 III p. 560; I754 p. 44.

796 Slabber, Martinus. 3r. X. I740 Middelburg-'s Gravenpolder 30. IV. I835. Burgomaster Goes, Administrator of Baarland, Bakendorp, Oudelande. Later Receiver of taxes for part of Zuid-Beveland (settling in 's Gravenpolder). Nagtglas I888-'93, IV, p. 634 (b); Van der Aa I852-'78 X (b); Museum of stuffed animals, especially birds, in 1824 sold to the Leiden 
Museum (Verhandeling over het opzetten van vogels 1816 ). Letters M. S. in Zeeuwsch Genootschap. He published: Natuurkundige Verlustigingen, Amsterdam I778; also in: Werken Zeeuwsch Genootschap I and following and in: Verhand. Holl. Mij X, I768.

797 Smit, D. Amsterdam. Cabinet sold 1761 (Vosmaer I790). cf. 805.

798 Slosser, see Schlosser.

799 Sluiter, T., Amsterdam. Broker. Cabinet divers naturalia sold 1757 (Vos. maer 1790).

800 Sluijter. Cabinet mentioned in Catal. Leers, p. IX.

80I Smit collection of Coleoptera given to Zoological Gardens Amsterdam (Guide Natura Artis Magistra I864 p. 25).

So2 Smit, D. Amsterdam. Cabinet sold i76r (Vosmaer 1790). cf. 805.

803 Smit, Daniel DE. Middelburg. Cabinet of shells. Valentijn I726 III p. 563, I754 p. 47.

804 Smit, H. G. DE, 's Gravenhage. Shells. R.

805 Sмiтh, Diderick. Merchant. Amsterdam. Argenville I757 p. I49; I780 p. 350 ; Cabinet sold I3. VII. I76I seq. (shells etc.) (Lugt I938 $\mathrm{n}^{\circ}$ I I66). cf. 802.

806 Smith, see Chatelain.

807 Smits van Burgst, C. A. L. Ginneken. Hymenoptera. Everts 1922 p. XII. R.

808 Smour, Jan. Captain of a ship, showed a rhinoceros in Amsterdam I74I (Witkamp 1864 p. IIO).

809 SNel, Jan. Rotterdam. Cabinet (v. Gool I750-'5I p. 339: possessed the insect-drawings of $\mathrm{K}$. B. Voet the elder).

810 Snellen, J. Dordrecht. Cabinet of shells, insects and other animals (Argenville I780 p. 338). (See following).

8I I Snellen, Johan. Rotterdam (same as preceding?) Pallas I766 1 p. 319 (Boddaert I768 p. 394); I767-'77 IX p. 5. Cabinet sold Rotterdam I787 (Vosmaer I790).

812 Snellen, Pieter Cornelis Tobias, 30. VIII. I832-29. III. I9I I. Rotterdam. Merchant. Wulp I895 p. I 5 (president Dutch Entom. Soc. I889). Published a catalogue of Dutch Lepidoptera 1867 and I882. Sepp I762-I860 VIII p. III, 43, 47, II9, I23, I30; Horn \& Kahle I935-'37 p. 262 (Lepidoptera in Mus. Leiden). M.S. in E; G p. 169.

S13 Snellen van Vollenhoven, Mr. Samuel Constant. Rotterdam 18. X. i81622. III.. I880. Conservator for entomology Museum Leiden (1854) President Dutch Entom. Soc. I852-'72 and 1878-'80: Wulp I895 p. 70 a.o.; Tijdschr. v. Entomol. XXIV p. LXXXIX (b, portrait works), portraits also ibid XIV. He gave the continuation of Sepp 1762-I860 (see VII p. I06, I26, I82, VIII p. IV, 58). Sale catalogue of his books 8. XI. I880 in E. Horn \& Kahle I935-'37 p. 262 (collections to Mus. Leiden, or via Ent. Soc. to Wageningen); G p. 270.

814 Snijder. Lepidoptera to Mus. Leiden. Lempke I936 p. 239. 
8 is Snouck Hurgronje. Collection of shells to Zeeuwsch Genootschap (Gedenkboek I9I9 p. I04). N. B. the Adriaan Isaac mentioned there are probably the christian names of Snouck Hurgronje, as these of ten appear in the family.

816 Snouckaert van Schaubburg, Mr. Dr. René Charles Edouard Georges Baron. 's Gravenhage Io. V. 1857-Territet 20. VIII. 1936. Collections of birds (stuffed by K. H. ter Meer). About 1900 he sold his stuffed birds to Zoological Gardens Amsterdam and to America and collected only skins, which collection was sold afterwards, partly to the Amsterdam Zoological Museum, and partly to Messrs Sillem and van Marle. He founded Igor the Dutch Ornithological Society with others and was its president I90I-'II. Disagreeing about the free collecting of birds he founded the Club van Nederlandsche Vogelkundigen (president till 1930). See Scheygrond in : Orgaan der Club v. Ned. Vogelk: IX 2-3, Nov. I936 p. 97 (b) with portrait.

817 Soemmering was a well-known anatomical preparator buying e.g. on the sale of Prof. van Geuns I3. VI. 1796, where also anatomical preparations of his hand were sold.

818 Soestdijk. During some time there was a menagerie near this Palace of the Stadhouders (e. g. under Willem III 1675, see Loisel I9I2 II p. 29; Tessin I9I4 p. 70 v 1687 also mentions a menagerie). King Louis Napoleon here commenced his menagerie (under Alpy, supervision of Reinwardt) which later went to Haarlem and Amsterdam (Witkamp 1864). In I810 Haupt (I8I4 p. 122) praises the game there.

819 Son, J. van. Collection of shells. Given by his brother P. and his nephew W. to the Zoological Gardens Amsterdam 20. X. 1904.

820 Sonnenberg-Gallant. Amsterdam. A painter who for $\mathrm{I}-6$ ducates stuffs birds, sells them and has a cabinet of his own. Witkamp I864 II p. III; Nemnich I809 p. 369 (v I808); Haupt I8I4 p. 338, 339 (v I8Io).

821 Spex, Jacob “Klerk ter Stadhouderlijke Secretarije”, 's Gravenhage. Cabinet of shells, fossils etc. sold I6. X. I776 ("ten huijse van den Raadsheer van Nispen op de Paviljoensche Gracht") (B $n^{\circ} 423$, p. I40; Lugt $1938 n^{\circ}$ 2593 ;. Vosmaer I790).

822 Stadhouder, see Oranje.

823 Stärcke, Dr. Aug. Psychiater, den Dolder. Formicidae. R.

824 Statius Muller, Prof. Dr. Ph. L., Prof. Hist. Nat. etc. Erlangen was a Dutchman. Many animals from his Cabinet in Knorr, Deliciae Naturae, which work he translated in Dutch (Dordrecht $177 \mathrm{I}$, see A XII, XIII, XIV, XXI, B IV-VI, C 5, D III, F IV-VI, G III-IV, H V-VII, K I-III, V, L II-IX, XI).

825 Stavoren. In this town Uffenbach I754 II p. 347 (v I7IO) saw a stuffed crocodile in the church.

826 Stautenberg. Shell-dealer in Amsterdam (Titius I783 IX p. I9I v I777). 
827 Steengracht, Cornelis, "Heere van Weehl, Slangenburg, Oost- en WestSouburg, Gedeputeerde wegens de Provincie Zeeland in de Generaliteitsrekenkamer". 's Gravenhage. Cabinet of all sorts of animals, sold I5. IV. I782 (B n ${ }^{\circ} 456$ p. 48 ).

828 Stel, Adriaan van DER. "Extraordinaris Raad van Indië, Landvoogd van Amboina" had a cabinet of shells (Valentijn I726 III p. 560, 563; I754 p. $13,19,44,47,62$ ). See also Houttuyn I76I-'73, I I6, p. 73 ; Kalff I928 p. 206.

829 Stem, van der. Amsterdam. Cabinet. Kanold 1727 p. 22.

830 Steijl. (Tegelen, Limburg). Missie-Museum possesses stuffed birds, shells, insects etc. (Musea, I938 p. $255 \mathrm{n}^{\circ}$ I98). Everts 1922 p. XII. Here Br. Berchmanns is a well known entomologist.

83 I Stilting, Physician. Utrecht. Cabinet (Boddaert iz7o V p. 29).

832 Stilting, I. Cabinet sold Amsterdam I77r divers naturalia (Vosmaer 1790) (the same as the preceding?).

833 Stoll, Caspar (Gaspard Stol). "Commies ter Admiraliteit" Amsterdam. Argenville I780 p. 354 (insects) ; Cramer I779-' 82 I p. 5, 6, 8, I2, I6, 51, 54, 70, 71, 106, I08, I12, I21, I22, I3I, I 50, II p. 8, 21, 35, 48, 83, 86, III p. 3, 8 , I7, 33, 43, 46, 47, 50, 52, 57, 66, 68, 70, 71, 75, 78, 79, 88, 93, 100, 102, IO9, I IO, I I I, II2, I I4, I I 5, I I 8 I I I53, I59, I60, I63, IV p. I, 9, I I, I2, I5, I6, 20, 25, 27, 28, 32, 36, 4I, 43, 46, $47,50,65,67,72,76,77,87,96,97$, I00, I18, I21, 137, I40, I45, I48, I64, I66, I69, I7I, 180, I93, I98, 201, 209, 214, 218, 221 ; Sepp 1762-1860 III p. I 18 , VI p. 9I. He edited himself Stoll $1788_{1}$, I788, and 1813. Maitland I855 p. I68.

834 Stoopendaal, Daniel. Cabinet sold Amsterdam 3. IV. I797 seq., divers naturalia (Lugt $1938 \mathrm{n}^{\circ} 5570$ ).

835 Storm, Joannes. Gardener of the Hortus medicus in Amsterdam (1750I803) and his wife had a cabinet with stuffed birds, butterflies and other insects in the chief building and a smaller cabinet of birds in a gardenhouse near the Muidergracht (Wagenaar, Amsterdam in zijn opkomst... I765, II, p. 387).

836 Stoutenburg, Theod. Joh. Cabinet of shells sold 27. VIII. 1799 (Lugt I938 $\left.n^{\circ} 5968\right)$.

837 Strohmeijer. Dealer in Naturalia. Amsterdam (Titius I783 IX p. I9I v I777).

838 Stroo, Cornelis. Alkmaar. Broker. Cabinet of nat. hist. sold 29. VII. I8I I (Lugt $1938 \mathrm{n}^{\circ} 805^{\mathrm{I}}$ ).

839 Struyck, Painter. Amsterdam. Cabinet. Houttuyn I76I-'73, I 9 p. I46, 526; Hagen 1862 II p. 394 mentions : Verschijden uijtlandsche Insecten, geteekent na het Cabinet van de Hn Seba, J. ten Kate etc. door Struijck, 6 vol., gr. $\mathrm{f}^{\circ}$. I718. This work appeared again in T. O. Weigels Catalog XIII 1862 p. 48 , but could not be traced further.

840 Suchtelen, see Sugtelen. 
841 Sugtelen, van. Burgomaster of Hoorn. Cabinet. Valentijn i726 III p. 562, I754 p. 46,48 , says it passed in the hands of Beudeker.

842 Superville. Cabinet of stones, minerals etc. etc. sold the Hague i748 (Vosmaer I790).

843 Suringar, Prof. Dr. Gerard Coenraad Bernhard. Lingen 8. IV. I802-Leiden I2. I. 1874. Prof. anat. chirurg. physiol. Amsterdam (1830). Prof. med. Leiden (I843-'72) (Horn I83I I p. 367 v I830). Murray I904 III p. 201 mentions two catalogues: one of anatomy, the other of cranes which he gave to the Leiden Academy, dated resp. I866 and I876.

844 Swammerdam, Jacob, Janszoon, father of following. Amsterdam 3I. I. 1606 - I678. Druggist in "de Star" (Oude Schans near Montalbaentoren, see van Seters, in De Groene Amsterdammer 15. II. 1930; Schierbeek in: Ned. Tijdschr. v. Geneesk. 8I, Io, 6. III. I937 (also b); Cole, Annals of Science II 1937). He had a large cabinet of which we possess different descriptions: the rare: Catalogus musei instructissimi, sale catalogue (Latin and Dutch) I679 [A, UBA], Vosmaer I790, Lugt $1938 \mathrm{n}^{\circ} 6$; Horn \& Kahle I935-'37 p. 273; Murray I904 I p. I I I, III p. 202 ; contains objects from all departments of nature and art fossils, vegetabilia, corals, birds, crabs, echinoderms, insects, fishes, mammals, serpents, shells etc.; in Recueil des voyages de M. Thévenot (ed. of I68I!); Valentinus 1726 III cap. I9 p. 458, Appendix von Kustkammern p. I9; Act. med. Hafn. III Obs. IV p. 8, LV p. 87, according to Uffenbach I754 III p. 5I0, 688, 689; See also: Boccone I674 p. 36, 278 (v I673-'74); Fuchs I916 p. 6, 3I (v I663); Jacobaeus I9Io p. 80 (v I674); Brown I682 p. 23 (v I668); Monconys I666 II p. I7I (v 1663); Hoogewerff I9I9 p. XVIII, XLIX, LXI, 369 noot 2, 39I-2 (v r667); Kanold I727 p. 22, I82.

845 Swammerdam, Jan. Amsterdam, i2 II. I637-17. II. 1680, son of preceding. Dr. med. (I667). His constitution was weak so that he never practised as a physician. Devoted himself wholly to scientific investigations, especially on insects. Lived with his father, but had a cabinet of his own. The Grand-duke of Toscane offered $f(200$ for it, if he himself followed thither, but Swammerdam refused (1668). When lateron (1675-76) he hoped to live in silence he tried in vain to sell it. He had taken the resolution to sell the cabinet in May 1680 when he died in February. Boerhaave in his Preface (b) to the Biblia Naturae of Swammerdam (1737-'38, 2 vol.) says nothing about the cabinet. Thevenot got the manuscripts and heiress was Margarita Volckers, wife of Dr. med. Daniel de Hoest. Whether she got the cabinet? His relation to her is not clear; Collot I814 VII p. I87, I90, I92, I94, I98, 40I ; Van der Aa I852-'78 X (b) ; Molhuysen I9I I-'37 IX p. IO2I (b) ; Harting in: Album der Natuur I876 p. I (b) ; Lidth de Jeude. Oratio... 21. I. I8I9 (A, UBA) ; Von Baer, Reden... I864; Duncan, Intr. t. Entomol. I840 p. I7; Gedenkboek 1932 p. 250; Blankaart, Schouburg p. 28, 31, IOI, I I7, I22, I42, I49, I56, I72, 175, I94, 
Collect. Medico-Phys. p. 29; Wulp I895 p. 72 ; Vandevelde in Verslagen en Meded. Kon. Vlaamsche Akad. v. Taal en Letterk. April 1930; id. in: Jaarb. Dodonaea IV 1937 p. I29 (b) ; Dict. Sc. Nat. 6 p. 222 ; Kerbert, in Bijdr. t. d. Dierk. Feestnr. I888 p. 5; Uffenbach I754 III p. 688; Maitland I855 p. I62; Sinia in Isis IX I880 p. 33 ; id. Dissert. I878 (b); Stoeder, Gesch. Pharm. Nederl. I891 p. 89, I65; Jöcher, I75I Allg. Gel. Lexic. IV p. 950; Banga, Gesch. Geneesk. p. 445, 447, 518, 546, 56r, 577, 599; Van der Boon, Gesch. ontd. ontl. v. d. mensch, I849 p. 50; Jacobaeus I9ro p. 80 (v I674); Brown I682 p. 23 (v. I668); Boccone I674 p. 278 (v I673'74); Boehmer I785-'89 II p. I23, 526, 27I ; Lesser I756 p. 86, 91, 64I ; Valentijn I726 p. 56r, 563; I754 p. 45, 49; Rumphius r705 p. I08; Herdenking van J. Swammerdam's 200-j. Sterfdag, I7. II. I880, Gen. Bev. Nat. Gen. Heelk. A'dam; Cole in Nature Febr. 6. 1937, p. 218, Febr. 13. I937, p. 287, Ann. of Sc. II 1937. Molhuysen 191 I-'37 X Kol. 995 (b).

846 Swart, Steven. Cabinet nat. hist. Sold 15-22. V. 1794, Amsterdam (Lugt I938 n $^{\circ}$ 5189).

847 Swinden, Prof. Dr. Jan Hendrik van. 's Gravenhage 8. VI. I746-Amsterdam 9. III. I823 Prof. Phil. Franeker (1767), Amsterdam (1785-1819). Cabinet of shells, minerals sold 9-I2. I1I. I824 Amsterdam (Lugt 1938 $\mathrm{n}^{\circ}$ I06II). Gedenkboek I932 p. 687 (b) etc.; Van der Aa 1852-'78 X (b). Molhuysen I9I I-'37 IV Kol. I289 (b).

848 Swinderen, Theodorus van, Groningen I4. IX. 1784-II. IV. I85I. Prof. hist. nat. etc. Groningen (18I5-'5I). Album Acad. Groningen... p. 623 (UBA) ; Academia Groningana I6I4-I9I4 p. 3I, 40, 4I, 50-54 (UBA). Van der Aa 1852-'78 X (b); Collot 1844 VII p. 287; Hoffmann I868 I p. 26r, II p. 337 (v I836) ; Mac Gregor I835 I p. 252 (v I833); Dethmar I838-4I III p. 238 ( $v$ I835-37). For us he is especially interesting because of the large Museum of Nat. Hist. which he founded (see Groningen). Murray 1904 II p. 268. Molhuysen I9I I-'37. II Kol. I4Io (b).

849 Taelman Kip, W. F. Cabinet of nat. hist. sold Amsterdam I6. III. I80I with others (Lugt $1938 \mathrm{n}^{\circ} 6216$ ).

850 Tarelinck, Christiaan van. Cabinet of nat. hist. Sold Amsterdam i8. VIII. I798 (Lugt I938 n $^{\circ}$ 5799).

85 I Tebure, see de Boer.

852 Tемminck, Jacob. Amsterdam. "Thesaurier der O. Ind. Cie"., lived Heerengracht near Leidschegracht, where he had his collection of birds. Nozeman I770 I; Thunberg I792-'94 I p. 68 (v I77I); Forster I868 p. 87 (v I790); Le Vaillant Voyage dans l'intérieur de l'Afrique... 2 tom. (UBA), Paris, I790 I p. I (v I780), Id. II. Second voyage dans l'intérieur de l'Afrique... Paris, an IV (UBA). p. 373 (v I784). Molhuijsen 19I I-'37 V Kol. 897 (b) ; G p. 45,267 etc.

853 Temminck, Coenraad Jacob. Amsterdam 31. III. 1778-30. I. 1858. Son of Bijdragen tot de Dierkunde, Afl. 27 
preceding, continued the cabinet. He often stuffed the birds himself and had a special method to prepare fishes. House quite filled with birds. In \pm 1820 he sold his collection to the Government for the Museum Leiden, of which he became the first director. Levensberigt by W. Vrolik in Versl. Kon. Akad. Amsterdam, afd. Nat., Gewone verg. 27. II. I858 (b) Collot I844 VII p. 243; Witkamp I864 II; Konst en Letterbode 1858 p. 67; Van der Aa 1852-'78 XI b; Jaarboekje Natura Artis Magistra I869 p. I93; Nemnich I809 p. 27I, (v I808); Haupt I8I4 p. 337 (v I8Io); Meiszner I8I9 p. 66 ( $v$ I8I7, birds, mammals esp. apes); Fliedner I83 I I p. 253 ( $v$ I823, shortly afterwards, he says, sold to University Leiden). In 1807 he edited: Catalogue systématique du cabinet d'ornithologie et de la collection de Quadrumanes de C. J. Temminck. See also the sale catalogue of his library 27. IX. 1858 (in E) $n^{\circ} 583-5$. And also Temminck I808-'o9 I, 28, 30, II 14, III, 2, I0, 12, I4, I9, 21, VI 58, VII 72, VIII p. 74, 76, 79; Stresemann I923 p. r20; Molhuysen I9II-'37 IV Kol. I299 (b) ; G p. 32, 45 a.o.

854 TEN ..., see ... (e.g. ten Hove, see Hove).

855 TER ..., see ... (e.g. ter Haar, see Haar).

856 Teroede, Amsterdam. Cabinet of shells. Valentijn i726. III p. 56r, i754 p. I2, 45.

857 Terschelling, West. Near the lighthouse "Brandaris" there is a Museum of birds that were killed when flying against the tower, blinded by the light (Musea 1938 p. $283 \mathrm{n}^{\circ} 228$ ).

858 Tesch, Dr. P., Haarlem, Coleoptera, Everts 1922. p. XII R.

859 Teyler, see Haarlem.

860 Thierens, see Witte.

86ı Thijsse, Dr. Jac. P., Bloemendaal. Born 25. VII. I865. Propagated the popularization of nature study. (Gedenkboek, De Levende Natuur, 25. VII. I935), collected all sorts of animals.

862 Tilburg. Natuurhistorisch Museum (Musea I938 p. $259 \mathrm{n}^{\circ} 203$ ).

863 Timmermans, Mej. Dr. A. Oegstgeest. Shells. R.

864 Toвias, D. Cabinet sold 21-22. IV. 1885 in Amsterdam, see: Catalogue de la préc. et sup. coll. de Coquilles etc., formée par M. Lyonet et augmentée par M. D. Tobias [A], bought by Museum Leiden.

865 Toorn, Mrs. A. A. J. H. van Der, Arnhem. Collection of shells to Leo Muller. R.

866 Tour, Baron Du. Cabinet of birds sold 's Gravenhage r776 (Vosmaer I790).

867 Toxopeus, Dr. Lambertus Johannes. Born 1893. Collection of insects, especially Lycaenids. Studied in Amsterdam, now secondary teacher in E. India. Horn \& Kahle 1935-'37 p. 28I.

868 Trap, P. W. M. Leiden. Insects. Sepp I762-1860 VI p. 53, 73, 103, 149, I60, 165, I69, I83, 195, VII p. 25, 29.

869 Trembley, Abraham. Genève 3. IX. I700-12. V. 1784. Studied in Leiden and 
was some time (I740-44) governor to the children Bentinck on Sorgvliet. Here he wrote his Mémoire pour servir à l'histoire d'un genre de polypes d'eau douce (1744). He probably collected nat. hist. like Lyonet, who engraved the plates for him. Collot I844 VII p. 204-5. Lamy I930 p. 6.

870 Treuer. "Gesandter der Baadensche, Darmstädter und Anspacher Höfe" 's Gravenhage. Sander I783 I p. 486 (v I777) (is this envoyé Truyer?, see. there).

87 I TRICHT, Barend van. Insects, Coleoptera (1922 to Mac Gillavry, Horn \& Kahle 1935-'37 p. 282).

872 Trotz, Prof. Mr. Dr. Christianus Henricus. I703 Kolberg-17. VI. I773 Utrecht. Prof. jur. Franeker I74I. Lived his last years in Utrecht ("pone Templum S. Petri") where his cabinet, with all sorts of animals, was sold 26. IX. 1774 (Catalogue B n 407 p. I35) Van der Aa 1852-'78 XI.

873 Truyer. Envoyé, 's Gravenhage. Cabinet of shells sold I783 (Vosmaer I790) (cf. Treuer).

874 Tutein Nolthenius, P. Insects. Horn \& Kahle I935-'37 p. 284 (to Lycklama à Nyeholt, Coldewey and Entom. Lab. Wageningen).

875 Tuyll van Serooskerken, F. L. S. F. Baron van. Macrolepidoptera to J. Th. Oudemans. Horn \& Kahle 1935-'37 p. 284 .

876 Twenr, M. Cabinet sold I789 Leiden (Vosmaer I790).

877 UbaGhs, Casimir. Maastricht. Fossils. Catalogue Liége r885 (A).

878 UijtTenbogaART, see also Utembogart and Uuttenbogaert.

879 Uijtrenbogaert, Dr. Daniel Louis, born 1872. Rotterdam, now Heemstede. Coleoptera. Everts I922 p. XII. R.

880 Utembogart, Christiaan. Cabinet. Patin 1676 p. I54 (v I67i).

88I UTRECHT. In this town the old travellers always went to the "English church" or "Collegiate church of St. Mary" to see the elephant's tooth and the 3 horns of the unicorn each about 3 feet long, hollow, out of which one could drink, that were shown there in the Clerk's House or library: Joly I672 p. I48 (v I646); Brown I682 p. 26 (v I688); Veryard I70I p. 6 (v I682); Mountague I696 p. I97-8 (v I695); Beckmann I912 p. 427 (v I762, could not see them).

The anatomy-hall of course contained a collection of anatomy and natural curiosities. Dryander I798-r800 I p. 223 gives a catalogue in fol. 1707: Nicolas Chevalier. Description de la chambre de raretez de la ville d'Utrecht, which probably is this collection, visited by: Bocage I764 III p. IO2 (v 1750); Ferrner I9I0 p. 397, 429 (v I759); Lynar I78I p. I39 (v 1771); Sander I783 p. 59I (v I777); Schaeffer I794 (v I788); Ireland I790 p. I75 (v I789); Thouin I84I p. I55 (v I795); Huber I8II p. I22 (v I809); Haupt I8I4 p. I02 (v I8Io); Anonymus I8I9 p. 2I I (v I8I9); Fliedner I83I p. 4 I8 (v I823-'24); Jaeck I824 p. 244 ( $v$ I824, refers also to cabinet of the town-druggist); Horn I83I p. 38 I, 383 (v I830); Dethmar 
I838-'4I p. 65 (v I835-'37); Guislain 1842 p. 89-90 (v I84I); Czermak I879 p. I68 (v I850). See also Terwen I855 p. I27. In the later years Bleuland gave a catalogue (Murray 1904 III p. 228) Descriptio mus. anat. I826, and Icones anat., $4^{\circ}, 1826$. Kernkamp editing Fermer I9ı gives p. 397 in a note interesting informations about the time 1759; Loncq, Histor. schets Utr. Hoogesch. tot I8I5 (Utr. I886); Muller. De Universiteitsgebouwen te Utrecht. 1899 .

Thanks to the activity of Lidth de Jeude Utrecht had a zoological garden (later "Tivoli"). Terwen I855 p. I28-9; Witkamp I864 II p. 27; Dethmar 1838-'41 p. 60-6I (v I835-'37); Guislain I842 p. 93 (v I84I). The zoological institute was and is housed in the ancient "Statenkamer", Janskerkhof and of course a zoological collection was made here. It was visited 1850 by Czermak (I879 p. 164) and 1879 by Blasius (I880 p. 26). See also Terwen 1855 p. 128 etc.

Of course the Veterinary school had and has cabinets of its own. Horn I83I p. 381 (v 1830); Dethmar I838-'4I IV p. 73 (v I835-'37) and Guislain 1842 p. 90 ( $v$ I84I) mention them.

We may finish with: Museum ten dienste van het Onderwijs, founded I9I6 (Musea 1938 p. $267 \mathrm{n}^{\circ}$ 2I I) and Museum van het Staatsboschbeheer (Musea $193^{8}$ p. $272 \mathrm{n}^{\circ} 215$ ).

882 Uuttenbogaert, Johan. Amsterdam. Since 1638 "Ontvanger der gemeenelandsmiddelen" (Etched by Rembrandt I639). Cabinet of shells, minerals and pictures. Hoogewerff I9I9 p. XXV, 63, 64, 81, 83 (v I667-'68). Elias. De vroedschap van Amsterdam I p. 26r.

883 UuttewaAl, Dr. J., Voorst. Insects. Sepp i762-I860 VIII p. 9I, IOI, I30.

884 V. D. V. Initials of a collector (animals in liquor, insects) whose cabinet was sold I. X. I777 in Utrecht. (Cobres I782 I p. I46).

885 VaArt, H. van Der. Santpoort. Coleoptera. Everts 1922 p. XII. R.

886 Valck Lucassen, Fr. F., Vorden. Coleoptera. Everts 1922 p. VII. R.

887 Valckenier, Wouter. Amsterdam "Bewindhebber Oost-Ind.-Cie." Cabinet (about I704). Valentijn I726 III p. 560; I754 p. I9, 44, 45.

888 Valckenier. 's Gravenhage. Cabinet Uffenbach I754 III p. 378 (v I7IO). 889 Valkenier, Pieter. "Gewesener Holländischer Abgesandter in der Schweiz". Lesser I744 p. 53; I756 p. 7I.

890 Valentijn, Ds. François. Dordrecht I7. IV. I656-6. VIII. I727. Clergyman Ambon (1686), Banda (was in Dordrecht 1695-1705), Batavia, Ambon (I707-r712), Dordrecht I714-'27 (some time 's Gravenhage). He wrote the large compilation Oud en Nieuw Oost Indien (1724-'26), in which we find so many of the old cabinets (Valentijn 1726, 1754). He was an eager collector himself as we infer from his work i726 III p. 560, 563, 578,$583 ;$ V p. 20 , II 3 seq. See Lesser 1744 p. 38, I756 p. 4I ; Van der Aa I852-'78 XI (b); Molhuijsen I9II-'37, V Kol. 989 (b); Collot I844 VII 
p. 313, 318; Rumphius Gedenkboek I902; Encyclop. v. Ned. Indië, 2e dr. IV I921 p. 5OI-8; Kalff in: De Indische Verlofganger III $n^{\circ} 27,1925$, p. 213-4; and in: De Indische Gids 22, 1900, II p. 907-939.

89i Van... see... (e.g. Van der Meulen, see Meulen).

892 Vanhoú́, see Hoeij.

893 VARI, Lajos, born 27. IX. I9I6 Budapest. Preparator Zoöl. Mus. Amsterdam. Lepidoptera $\mathbf{R}$.

894 Vecht, J. VAN DER. Insects. Horn \& Kahle I935-'37 p. 288 (I928 to Mus. Leiden) $\boldsymbol{R}$.

895 VeEn, Hendrik I85I-I926. Insects. Horn \& Kahle I935-'37 p. 288 (to Kol. Inst. and Mac Gillavry).

896 VeEn, Pieter. Haarlem. Insects. Sepp I762-I860, III p. I42, I54, I59, I62, IV Preface, p. 2, 16, 20, 21, 30, 50, 74, 102, I1 I, 136, V p. I 54

897 Velde, van DE. Insects. Cabinet sold Utrecht 1777 (Vosmaer 1790). Cramer I779-'82 II p. II.

898 Velden, Jacob van De. Cabinet (nat. hist.) sold 3. XII. I781, Amsterdam (Lugt $1938 \mathrm{n}^{\circ} 3327$ ).

899 Velten, see Blauw Jan.

900 Vereijle. Amsterdam. Cabinet of shells. Argenville I780 p. 356.

901 VerhaAr, C. P. D. 's Gravenhage. Shells. R.

902 Verheijk, Dr. Hendrik. Physician. Rector of the Lyceum Amsterdam. Cabinet, especially of Birds. Argenville I780 p. 335; Nozeman I770 I; Björnståhl I782 p. 46I (v I775).

903 Ver-Huell, C. J., Doesburg (Huize Kemnade) Insects. Sepp 1762-1860 V p. $18,36,56$.

904 Ver-Huell, Quirijn Maurits Rudolph. Zutphen II. IX. I787-Arnhem Io. V. I860: Rear-admiral. Excellent draughtsman. Van der Aa 1852-'78 XI (b) ; Alg. Konst en Letterbode I860; Tijdschr. Ned. Entom. Vereen. IV p. 55, XI p. I89, I92 (b) ; Drawings in E; Wulp I895 p. 32 ; Eijndhoven I86I p. 46, n० I22; Sepp I762-I860 V preface p. 7I, 80, 87, I03, I I 5, I 22, 124, I36, I 50, VI, preface p. 2, 34, 39, 42, 46, 49, 62, 65, 71, 81, 102, I03, I I4, I I 7, I26, I27, I33, I39, I42, I74, I79, VII p. II, 12, 33, 52, 97, I02, I I3, 143, I5I, I66, I68, I8I, 197, VIII p. III, I5, 18, 58, 62, I19; Molhuijsen 19II-'37, III, Kol. I29I (b). Insects to Leiden Museum (G p. I70).

905 Verloren, H. (later Verloren van Themaat), Utrecht r. IX. I8I4-22. II. 1885. Jur. cand., Utrecht. Cabinet. Sepp I762-1860 V p. I36, VI p. I00I02. Album Studiosorum Rhen. Traj. p. 292 (I832).

906 Verloren van Themaat, Dr. M. C. Amersfoort. Insects. Horn \& Kahle I935-'37 p. 50 (sub voce Dalen). Cabinet sold I6. VIII. I938.

907 Vermande, A. and others. Some natural history sold 6.V. r8II. Haarlem (Ekama 1874 p. I03; Lugt $1938 \mathrm{n}^{\circ} 7985$ ).

908 Vermande, widow A., née de Waal. Cabinet sold 21. XI. I820. (Ekama I874 p. IOZ). 
909 Vermeulen. Amsterdam. Merchant. Cabinet of birds and insects. (Thunberg $1792-' 94 \mathrm{II}_{2}$ p. $257, \mathbf{v}$ 1779). Perhaps this is Willem van der Meulen (see there).

9 Io Vermeulen, (Mr. Pieter, Haarlem 4. II. I732-I2. VI. I81o. Burgomaster etc. of Haarlem, "Hoog Bailluw van Kennemerland" etc. Perhaps he is meant by): Argenville 1780 p. 357 . Boddaert 1768 p. XX also names a cabinet Vermeulen. See also Van der Meulen.

91 I Vermeulen, F. P., born 29. VII. 1870. IJmuiden. Secretary to the Society of fishery-ship-owners. Cabinet of zoology, especially shells. Presented many objects to Zoological Museum Amsterdam.

912 Vernhout, Johannes Hendrik, born 24-X-I866. Conservator Museum Leiden (1908-'16) and Zeeuwsch Genootsch. (Gedenkb. 1919; G p. 280).

913 Verrier, J. DU. Cabinet sold Rotterdam 750 (Vosmaer 1790).

914 Versluys, Prof. Dr. Jan, born Amsterdam I873. Prof. Zool. Vienna. Coleoptera Everts. I922 p. XII. R.

915 Vervel, Antoni, druggist, Dordrecht. Cabinet especially shells. Valentijn I726 III p. 563, 578; I754 p. 5, 26, 47, 62, 65.

916 Vescher. Amsterdam. Cabinet. Journal des Sçavans de l'an MDCLXXXI, Tome IX, Amsterdam I682 p. 49-5I. (Perhaps Visscher?).

917 Veth, Huibert Johannes, 1846-1917. Coleoptera, Everts 1922 p. XII; Horn \& Kahle 1935-'37 p. 289 (to Mus. Leiden).

918 Viandt. Cabinet mentioned in Velten M. S. \pm I700, (v I7OI).

919 Vincent, Levinus. Amsterdam 1658-Haarlem 8. XI. 1727. Merchant Amsterdam, later Haarlem. Married Jannetje van Breda, whose brother Anthonie van Breda had begun the collection which was so well-known in 1674. Se remarried "Baljuw" van der Mark at Warmondt. I may refer to the article of $\mathrm{Mr}$ Wijnman in Molhuysen 191 I-'37, X Kol. I I04-6 to which I had the pleasure to contribute, for the record of his own publications on his cabinets and also for the biographic literature. Since that time the following occurred to me: Lesser I744 p. 62; I756 p. 88; Cramer I779-'82 IV p. 67; Valentijn I754 p. I3, 43, 45, 49, 50, 5I, $52,63,64,65,66,67$; Maitland I855 p. 168; Argenville I780 p. 345 (Bäutt see Bout); Knorr I770 V p. 35; Petiver, Museum 1695-1703 p. 96. The collection came to Jhr. P. Bout (see there).

920 Vinne, Jan van Der. Haarlem 3I. I. I699-8. XI. I753. Painter. Cabinet sold i3. V. 1754. (Ekama 1874 p. 103; Lugt $1938 \mathrm{n}^{\circ} 837$ ).

921 VIs, Jacob. Shell-collection to Zoological Gardens Amsterdam.

922 Visscher, see Vescher.

923 Visscher, J. DE. Cabinet sold Amsterdam i769. (Vosmaer I790).

924 Visvliet, Mr. Egbert Philips van, 1736-'99 Middelburg. (Brakstraat). Dr. med. Cabinet of anatomy (p. 175) animals in liquor, shells (p. 188) zoophytes etc. sold $3-5 . \mathrm{X}$. 1799 in Middelburg (B $\mathrm{n}^{\circ}$ 677). Nagtglas I888-'93 IV p. 864. 
925 Vlaming, Cornelis DE. Returned I7I5 from Batavia with 400 drawings of fishes, called "Zeetooneel". Used by Ruysch in his edition of Jonston (I718), by Valentijn I726 and by Vosmaer 1754 when he edited Renard. (Six, Overzicht der diensten door het Rijksmuseum voor Nat. Gesch. te Leiden aan de Dierkunde bewezen).

926 Vlietstra, A., Beverwijk, Shells. R.

927 Vlissingen. Here the "Zeeuwsch Genootschap" was founded, see Middelburg.

928 Vlugt, Willem van Der. Haarlem Cabinet. Cramer I779-'82 I p. I29, II p. I3.

929 Voet, Carel Burchart. Zwolle 22. VI. I67I-Dordrecht 1745. Painter and "Commies ter Recherche" Dordrecht. Cabinet o fshells and insects. He also had birds, animals in liquor and minerals. He made drawings of several insects and their metamorphosis. Valentijn I726 III p. 562, 575 (d); I754 p. 5, 6, 23, 25, 27, 28, 46, 48, 59, 63, 64, 65, 66; Van der Aa I852-'78 XI (b); van Gool I750-' 5 I I p. 329; Maitland I855 p. I68; M. S. with portrait in E; Voet I806 I p. 47; Molhuysen I9II-'37 X Kol. II27 (b).

930 Voet, Mr. Dirk, son of preceding. Zwolle “Gemeensman", Cabinet, especially shells. Valentijn I726 III p. 563 ; I754 p. 47, 66.

93 I VoEt, Henrik, brother of Carel Burchart preceding, Zwolle, Burgomaster and Postmaster of Overijsel. Cabinet especially shells. Valentijn I726 III p. $563 ;$ i754 p. 47.

932 Voet, Joan Eugenius. Dordrecht 24. I. I706--28. IX. I778. Son of Carel Burchart preceding. Physician. Dordrecht. He also possessed a cabinet, especially shells and insects, about which he edited his Catalogus systematicus coleopterorum 2 vol. (Latin, French, Dutch 1766, with new title page I806). He mentions his own cabinet in I p. 27. When Valentijn left Dordrecht Voet took his place in the Dordrecht Malacological Society (Liefhebbers van Neptunus Cabinet), see Valentijn I726 III p. 563 ; He is mentioned also by Valentijn I754 p. 47. His shells were sold I779 in the Hague. (Vosmaer I790); Molhuysen I9I I-'37 X Kol. I I27 (b).

933 Voet, Dr. Carel Burchart, son of Joan Eugenius preceding. Physician. 's Gravenhage. He also had a cabinet especially insects, as his father mentions it: Voet I806 I p. 8, II, I6, I7, 20, 22, 23, 26, 31, 32, 46. See also: Houttuijn I76I-'73 I I3, p. I75-6; Argenville I780 p. 337 (elle embrasse les productions des trois règnes, mais les insectes s'y distinguent principalement), 346; Anonymus I767 p. 328; Cramer I779-82 I p. 5 Introd.; Pallas I767-'77 VJ p. 3, IX p. 83; Schaeffer I794 who visited the Hague I787-'88 mentions a cabinet of minerals of Voet; G p. 30.

934 Voigt, J. G. from Freiburg, was a dealer in naturalia in Amsterdam and was also a collector himself. Rudolphi I804. p. I I (v I802); Nemnich I809 p. 369 (v I808). He died 82 years old and his cabinet of shells was sold Amsterdam I9. X. I837 seq. (A); G p. 4I. 
935 Volkert (Janse) or Volkard Jansz., probably $\dagger$ ı68ı. Merchant Amsterdam (Nieuwendijk near Dirk van Hasseltsteeg). He had a cabinet which was visited by Knorr von Rosenroth (v I663), see Fuchs 1916 p. 5, II ; Boccone r674 p. 278 (v I673-'74); Valentinus I7I4 II p. 19.

936 Volkertsz (Volkaartsz, Volckers, Völckersen), Johann. Amsterdam. Cabinet especially shells, which he had etched by Savry: Valentijn 1726 III p. 561 ; 1754 p. 45 ; Kanold I727 p. 182 ; Rumphius I705 p. 67, I6I ; Uffenbach I754 III p. 688 (v 1710).

937 Voorhoeve, Mr. J. Rotterdam. Cabinet (containing collections of Scheepmaker) sold 2. IV. I872 (B 2255a).

938 Vos tot Nederveen Cappel, Lodewijk Hendrik Daniel De. I846-i934. Velp. "Griffier van de Staten". Cabinets of shells and insects, the first bought by Ir. H. G. J. Schelling, Utrecht, the insects (Horn \& Kahle I935-'37 p. 29I) to his son, (follows), Klijnstra, Valck Lucassen, Uyttenboogaart, Mac Gillavry, Leiden Museum (Lempke I936 p. 239). Everts 1922 p. XII.

939 Vos tot Nederveen Cappel, J. J. de, son of preceding. Velp. Coleoptera.

940 Vos tot Nederveen Cappel, H. A. de. Apeldoorn. Lepidoptera ẹtc. Everts 1922 p. XII.

941 Vosmaer, Aernout, Rotterdam 23. X. I720-'s Gravenhage I5. I. I799. He was Director of the menageries and of the "Natuur- en Kunstcabinetten des Stadhouders" which were founded by the Widow of Prince Willem IV in 1756 after she had bought Vosmaer's own cabinet (see s. v. Oranje). It was continued under Willem $V$, who was much interested in it and made Vosmaer "Raad" between I778 and I783. Vosmaer described many of the animals under his care in his work: Natuurkundige Beschrijving eener uitmuntende Verzameling etc. Amsterdam 1766-I804 (also French edition). They were mostly founded on animals that had lived in the menageries (see s. v. Loo) especially on "het Kleine Loo" near 's Gravenhage. The honours gradually bestowed on the author show how his scientific importance increased. There can be found much more about Vosmaer in the Koninklijk Huisarchief and in the Archives of the family Vosmaer in Leiden, as I know from kind informations of Mr. C. J. J. G. Vosmaer. Van der Aa I852-'78 XI (b) ; Collot I844 VII p. 246-'52; Konst- en Letterbode 25. I. I7.99, $\mathrm{n}^{\circ} 265$ p. 25 ; De Navorscher I857 p. I 59 (vr. 208, 308), $185^{8} \mathrm{n}^{\circ} \mathrm{I} 8 / 19$. Of course he was often visited, referring to: Oranje, I may add here: Ferrner I9Io p. 354, 495 (v I759); Garampi I889 p. 204 (v I76I '63) ; Beckmann I9I2 p. 344 (v I762, much important information!) ; Björnståh1 I782 p. 367, 4I6 (v I774); Grimm 1775 p. 290 (v I774); Honoré I779 p. 59 (v I776); Sander I783 I p. 486, 489, 49I (v I777); refers to Catal. Library of Crevenna, Italian Merchant in Amsterdam I775; Titius I783 p. 204-'I3 ( 1777 ); Forster I843 p. 88-9 (v I778). He is also mentioned by Strick I8I8 p. I97; Francq I769-I8I I III p. I80; Argenville 
I780 p. 338, 34I ; Pallas I766, p. XV, 158, I93 (Boddaert I768 p. XXXVI, I98, 240); $1766_{2}$ p. X, 5-6, I 18 (Boddaert I770 I p. VIII, IV p. 9); Houttuijn I76I-'73 4 p. 326,-I8 p. XI ; Van der Aa I852-'78 s.v. Jan van Lier; Loisel 1912 II p. 32, 34, 53, 303. Vosmaer often helped Meuschen in editing his catalogues (see ${ }^{\circ} 3$ Oudaan $\mathrm{I} 766$, which contains a Systema testaceorum of his hand p. 52, see also p. 53-57); he edited part III and IV of Seba's Thesaurus (1734-'65) as is certain through letters in the family archives between V. and Van Homrigh; the work of Renard, Hist. Nat. d. plus rares curiosités de la mer des Indes 1754 was edited through his care. Pallas i767-'77 VI p: I I quotes: A. Vosmaer, Geslagten der Vogelen Amsterdam $175^{8}$ in 8 vol.

The M. S. catalogue which Vosmaer made of the sales of Cabinets in his time is now in B (see also p. 248 of this paper). The sale catalogue of his books (in A, also Lugt $1938 \mathrm{n}^{\circ} 6039$ ) sold 17 . III-I. IV. I800 in his house (Hooge Nieuwstraat), shows us the richness of his library. We especially refer to p. $86 n^{\circ} 367,368$ (earlier sales? 1764 and 1765 ), p. $88 n^{\circ} 388$, p. 426,427 (his cabinets).

942 Vreeswijk, G. edited: Het kabinet der mineraalen, Amsterdam i67o continued 1675 (Vosmaer I800 p. 8I $\mathrm{n}^{\circ} 259$ ).

943 VRIENDS (Vrients), Bernhardus. Haarlem buried I2. V. I791, nearly 65 years old. Silk manufacturer Haarlem (Groote Houtstraat near Paarlaarsteeg). Cabinet of diverse naturalia of all classes sold 27. IX. I79I seq. (Ekama I874 p. 103). He is mentioned by Cramer I779-'82 I p. 36, 58, $86,87,94, \mathrm{I} 28, \mathrm{I} 44, \mathrm{I} 45, \mathrm{146}, \mathrm{I} 47, \mathrm{I} 49$, II p. 13, 16, 28, 29, 56, 75; Francq I73 p. 36 ; Argenville I780 p. 357 ; Sepp I762-1860 III p. II8 (butterflies went to C. van Lennep in I79I). Vriends himself buys on auctions Leers (through Meuschen) and Elout. He was visited by Sander I783 I p. 540 (v I776); Thunberg I792-'94, II 2 p. 257 (v 1779); Titius I783 p. 199 (v 1777 ).

944 VRies, Tsjeerd Gs. DE. Leeuwarden. Collection of birds and eggs. R.

945 VRIEs, J. C. DE. Lepidoptera (Horn \& Kahle 1935-'37 p. 292, now in Leiden Museum); G p. I7I. Probably the same as 946 .

946 VRIEs, J. DE. Cabinet mentioned in Sepp I762-I860 VI p. I77. See 945.

947 VRIESE, DE. On 20. III. I 863 shells were bought for the Zoological Gardens Amsterdam on an auction de Vriese. G. p. I7I (some birds to Leiden).

948 VRIJDAG, Daniel. Amsterdam. Cabinet sold I7-I9, 24. I. I825. (Lugt I938 in 10798).

949 VRoEg, Adriaan, 's Gravenhage. Cabinet of animals from all classes offered for sale 6. X. I764. He who wanted to buy the whole museum was asked to apply to $\mathrm{Mr}$ Vroeg, Vijverberg, the Hague. Pallas $1767-77$ informs us VI p. 12 that a part went to the Kabinet of the Stadhouder. Stresemann 1923 p. I20 quotes Pallas.

950 Vrolik, Prof, Dr. Gerardus, Leiden 25. IV. I775-Amsterdam Io. XI. I859. 
Prof. bot. anat. phys. Amsterdam (1797-1859). Gedenkboek I932 p. 55, 7I, 167, I85, I86 (portrait), 187, 268, 275, 704 (b) ; Van der Aa 1852-'78 XI (b); Molhuysen I9II-'37, III Kol. I366 (b) ; UBA H. S. Cat. II $\mathrm{n}^{\circ}$ I4I9; his books were partly sold 3 . XII. I860 (A), this catalogue contains a list of his works and a biographical note. He was visited by Rudolphi I804 p. I07-8 (v I802); Nemnich I809 p. 348 (v I808); Haupt I8I4 p. 337, II p. 99-IOI (v I8Io); Jaeck I826 p. 232 (v I824); Horn $1831-33$ p. 363,368 ( $v$ i83o!). After his death his anatomical and zoological cabinet came to his son (follows). With Reinwardt he had the supervision of the menagerie in the Hortus medicus (see Lodewijk Napoleon).

951 Vrolik, Prof. Dr. Willem, son of preceding, Amsterdam 29. IV. I80I22. XII. 1863. Prof. anat. Groningen (1829) Amsterdam (1831-'63). Gedenkboek 1932 p. 6I, 7I, I86 (portrait), I87, I89, 275, 704 (b); Van der Aa I852-'78 XI (b); Molhuysen I9I I-'37 III Kol. I367 (b) ; UBA H. S. Cat. $\mathrm{n}^{\circ}$ I396; Jaarboek Kon. Akad. van Wet. Amsterdam 1863 (b by Van der Hoeven); after his death his rich library was sold I6. I. I865 (with list of his works and biographical note, in A) and agin 15-17. IV. I868 (A). After his death Dusseau made a Catalogue of the collections G. and W. Vrolik. The anatomical part, known as "Museum Vrolik", is now preserved in the Anatomical Laboratory Amsterdam. The zoological part went to the Zoological Gardens Amsterdam. Kerbert 1926 p. 4 note 4 tells us that the collections were saved for the town by a committee of citizens for $f$ I2000.- Willem Vrolik has done much for the Zoological Gardens Amsterdam. He was visited by Arlincourt I842 II p. 49 ( \pm 1837$)$; Andersen I855 p. 407 (v I847, prof. Frøhlich); Czermak I879 p. I70 (v 1850); Blasius 1880 p. 14 mentions him.

952 Vroonhof, P., Cabinet sold 1773 Amsterdam (anatomy etc.) (Vosmaer I790).

953 WaAl see Vermande.

954 Wachendorff, Prof. Dr Evert Jacob, Utrecht 2. XI. 1703-22. XII. 1758, Prof. med. bot. chem. Utrecht 1743 . Cabinet, with 200 animals in spiritu and 32 dry animals, sold $I$ r. VI. 1759 seq. (B $\mathbf{n}^{\circ} 259$ ). He had lived Utrecht "Nieuwe Gragt onder de Linden" (In municipal library Utrecht a M. S. List of what was bought for Academy). Bijdr. Meded. Hist. Genootsch. 3I p. 404, 4I8, 427, 428; Molhuysen I9II-'37 V Kol. I038 (b).

955 Wageningen. In the collections of the "Landbouw Hoogeschool" a.o. the collection of insects of the early Entomological Society, also that of Snellen van Vollenhoven.

956 Walcheren, J. C. H. van. Brummen. Eijndhoven I86I bought butterflies at his auction, which after Eijndhoven's death were bought by Verloren. Sepp 1762-1860 VII p. 209, VIII p. I16. Horn \& Kahle I935-'37 p. 294. 
957 Walle, P. VAN DE. Cabinet of animals in liquor sold Amsterdam 1722 (Vosmaer I790).

958 Wasmann S. J., Erich, Valkenburg collected ants, termites and their guests. Everts 1922 p. XII; Lieftinck 1925 p. 67.

959 WassenaAr, Johan Hendrik van, Heere Van Wassenaar, Obdam etc. etc. 's Gravenhage, Cabinet of shells, zoophytes, minerals, art etc. Valentijn I726 III p. 562 ; 1754 p. 46 ; Argenville I757 p. I49; I780 p. 343 ; it was sold 25. X. 1769 seq. in Amsterdam and contained 860 nrs nat. hist. (Lugt $1938 \mathrm{n}^{\circ}$ I784; Vosmaer I790).

950 Water, Jona Willem te, Zaamslag 28. X. 1740-Leiden 19. X. I822 Prof. theol. et hist. Middelburg ( 1780 ) Leiden ( 1785 ). Cabinet nat. hist. sold 28. X. I823 Leiden (Lugt $1938 \mathrm{n}^{\circ}$ 10527). Collot I844 VII p. 279; Molhuysen I9II-'37 IV Kol. I438 (b).

96I Weber, Prof. Dr Max, Bonn 5. XII. 1852-7). II. 1937 Eerbeek. Prof. Zool. Amsterdam (I883-I922). Gedenkboek I932 p. 276, 285, 706 (b); de Beaufort in Vakblad v. Biologen XVIII 7, March 1937, p. I25 (b); id. in Tijdschr. Kon. Nederl. Aardr. Gen. (2) LIV, I937, 2 p. I53; d'Arcy W. Thompson in: Obituary Notices Roy. Soc. London $n^{\circ} 6$, vol. 2, p. 347-55, Jan. 1938; id. in Nature 1937. He raised the Zoological Museum Amsterdam to its present state (Kerbert 1926). He had a private collection of coleoptera. (Everts 1922 p. XII).

962 Weijenbergh, Dr Hendrik, I842-'85. Insects from Argentinia (Horn \& Kahle I935-'37 p. 301), M. S. in. E.

963 Wempe, H. E., Wormerveer, Burgomaster of Westzaan. Shells. R.

964 Werrently, Ds Georg. Henr., Clergyman in Batavia, later Amsterdam. (Seba 1734-'65 I p. 162).

965 Wertheim, A. C., 22. IX. I889-XII I932. His collections 1923 to Zool. Mus. Amsterdam.

966 Werumeus, Arnold Sicco. Collection nat. hist. sold Groningen ri. V. i702 (Lugt $1938 \mathrm{n}^{\circ} \mathrm{I} 82$ ).

967 Westerhoff, Dr. Abraham, 's Gravenhage Physician. Cabinet of nat. history, anatomy, zoophytes, shells, insects etc sold I8. IX. I782 in the Hague (in aedibus defuncti in platea “'t Voorhout") ( $\mathrm{n}^{\circ} 462$, Vosmaer I782).

968 Westerman, Dr Gerardus Frederik. Amsterdam 8. XII. I807-9. V. I890. Bookseller, birdcollector, founder of the Zoological Gardens Amsterdam. Eigen Haard I890 (b) ; Bijdragen tot de Dierkunde I 888 Feestnr. (Maitland p. 3) ; Abrahams en Portielje, Artisboek I922. II p. 294 ; Zool. Garten XXXI p. 255, Blasius I880; Het geslacht Westerman (b).

969 Westerveen, A., “Advocaat der O. Ind. Cie.", Cabinet sold Amsterdam r76r (Vosmaer I790). Valentijn I726 III p. 56r, I754 p. I9, 45; Catal. Leers p. IX.

970 Wichers, Jan Gerard, Governor of Surinam. He returned i79I to Holland 
(Jutfaas) with drawings of butterflies from Surinam which H. J. Scheller had made for him. Via J. Calkoen they came to Sepp who edited them as: Surinaamsche Vlinders I-III, Amsterdam.

97 I Wickevoort Crommelin, van, Haarlem i890. His collection of birds in Mus. Leiden ( $\mathrm{G}$ p. 163).

972 Wiel, P. van der. Amsterdam. Coleoptera. Everts 1922 p. XII. R.

973 Wijga, P. Medical Officer, once Ceram, ( \pm I896) now Amsterdam. Shells. R.

974 Wijnbelt, J. C. Amsterdam. Microlepidoptera. R.

975 Wijnpersse, Prof. Dr. Dionysius van De. Middelburg 18. III. I724-8. X. I 808 Leiden. Clergyman, Prof. phil. Groningen (I752), Math. Leiden ( 1769 - I805). Árgenville I780 p. 348 (cabinet ne consiste qu'en minéraux); Smith I796 I p. I I (collection of pathological bones); Pallas I766 1 p. 34I (Boddaert I768 p.422) ; Houttuijn I76I-'73 I I7 p. 83; Nagtglas I888-'93 IV p. Io09 (b) ; UBA H. S. Cat. II p. 201 n $^{\circ}$ I242; Van der Aa 1852 -'78 XII (b); Molhuysen I9II-'37. V. Kol. I I50 (b).

976 Wijnpersse, J. T. van. Cabinet of Anatomy sold Leiden 1788 (Vosmaer I790). Nagtglas I888-'93 IV p. I009 (b).

977 WILDE, Everhardus DE, "Chartermeester van het Collegie der Edel Mogende Heeren Raden van Staten". Cabinet of Natural History, sold at The Hague 7. IV. I763 (B).

978 Wilde, Iz. A. J. DE, born Poortvliet (Zeeland) I877. Headschoolmaster and teacher at secondary school Amsterdam. Shells. R.

979 Willem, Mrs DE, "épouse de M. Willem conseiller à la Cour de Justice de la Province de Hollande". Cabinet (Argenville I780, p. 347).

980 Willemse, Dr. A., Well (Limburg) Insects. Everts 1922 p. XII. R.

981 Willemse, C. J. M., Eijgelshoven (Limburg). Orthoptera. Everts 1922 p. XII. R.

982 Willemsen. Zutphen. Insects. (Sepp. I762-I860 VII p. III).

983 Wrllink, J., Winterswijk. Coleoptera. Everts 1922 p. XII. R.

984 Willink van Collen. Ladies. Bird collection in the castle of Gunterstein, Breukelen. Presented to Zool. Museum Amsterdam.

985 Wilmink, F., Leiden, Shells. R.

986 Wind, Dr. Paulus DE. Middelburg 8. V. 1714-r. II. I77I. Lector anat. Middelburg Cabinet of animals and anatomy sold 17. XII. I77I Middelburg (B 382). Nagtglas I888-'93 p. 977, 978 (b). His collection of “Stones" from the bladder etc. in Museum Zeeuwsch Genootschap.

987 Winterswijk. Het Museum (Musea r938 p. $285 \mathrm{n}^{\circ} 23 \mathrm{I}$ ).

988 Wisselingh, Ir. T. H. van. Heemstede, "Hoofdingenieur bij 's Rijks Waterstaat". Lepidoptera. Lempke I936 p. 239.

989 Wit, Willem DE. Amsterdam. Shells. R.

990 Witte Thierens, Henderick DE. "Eerste commies en kassier ten comptoir 
generaal van Zeeland". Cabinet of dried Zoophytes, like plants in a book p. $106 \mathrm{n}^{\circ} 9$ in his catalogue of sale Middelburg $\mathrm{I}-2$. VI. I763.

991 Witsen, Jonas. "Membre de la municipalité d'Amsterdam" (Valentijn writes: "geheimschrijver"). His cabinet is mentioned by Seba I734-'65 III p. 3I ; Valentijn I726 III p. 56I ; it was sold I6. VIII. 1790 in Amsterdam (Lugt $1938 n^{\circ} 4620$ ) (2e partie: Hist. nat. $192 \mathrm{~ns}$ ): (Was this Jonas Witsen, Amsterdam 6. VIII. I705-9. XII. 1767? See Molhuysen I9II-'37 IV Kol. 1473).

992 Witsen, Mr. Nicolaas, Corneliszoon. Amsterdam 8. V. I641-i717. Burgomaster etc. Wellknown for his connections with Czar Peter. Van der Aa I8: 2-'78 XI (b); Molhuysen I9II-'37 IV Kol. I473 (b); Greshoff in Llbum der Natuur I909 (! b); Gebhard, Leven van N. W. (uitg. Prov. Utr. Gen. I88I-'82) ; Jaarb. Amstelodamum XIII 1915 p. 65 ; Scheltema, Peter de Groote, 1842; Wagenaar, Amsterdam in zijn opkomst... III I767 p. 239; Raptschinsky 1936; Collot I844 VII p. I99; Pacichelli r685 I p. 639 (v I674); Patin I676 p. I6I (v I67I : M. de Witzen, sécr. de la Ville); Misson I722 I p. 34 (repeats Patin); Bowrey 1927 p. 4I (v I698); Uffenbach I754 III p. 687 (v I7IO); Jobi Ludolfi, Diss. de Locustis, pl. 20, grashoppers which Witsen gave him (behind Jobi Ludolfi, Historia Aethiopica, Frankf. a. M. I68I, UBA); Valentijn I726 III p. 56I ; 1754 p. 19, 45; Seba 1734-'65 I p. 53, 138. His cabinet (nat. hist. and minerals $425 \mathrm{nrs}$ and 42 drawers) was sold 30 . III. I728 seq. in Amsterdam (Lugt $1938 \mathrm{n}^{\circ} 368$ ).

993 Witsen. "Majoor", Amsterdam. Cabinet of shells (Valentijn I726 III p. 56I). 994 WITT, DE, "secretarius te Dordrecht" had a curious cabinet (naturalia?) Kanold 1727 p. 33.

995 Wittpen, J. H. E. Amsterdam. Lepidoptera etc. Everts 1922 p. XII, Lempke I936 p. 239. $\mathbf{R}$.

996 Woertman, J. J., Utrecht, "Schepen". Cabinet (nat. hist., anat.) sold Utrecht 5-8. III. I8IO (Lugt I938 $\mathrm{n}^{\circ} 7715$ ).

997 Wolff van Westerrode, Mr. de, Harderwijk “Overste-luitenant". Cabinet of insects. Sepp I762-I860 III p. 98, I47, I67, IV p. 26, I05, I I2, I I4.

998 Wolthers, Harmannus. Groningen. "amplissimo senatui civitatis Groninganae adscriptus, et, cum rebus humanis eximeretur, in Supremum Belgii Faederati concessum adlectus". His cabinet with many animals, shells, zoophytes etc. (p. 53) was sold 28. IV. --3. V. I789 Groningen (B $n^{\circ} 540$; Lugt $1938 n^{\circ} 4432$ ).

999 WTTENBOGaERT see Uuttenbogaart.

1000 WTteWAaL $=$ Uuttewaal.

1001 Wulp, Frederik Maurits van DeR, 's Gravenhage I3. XII. I8I8-'27 X. I899. "Referendaris bij de Rekenkamer". One of the founders of the "Nederlandsche Entomologische Vereeniging" 1845 and its secretary 1870-'94. He wrote the history of its first 50 years (Wulp I895; portrait); Tijdschr. 
v. Entom. I900 (b) ; M. S. in E. He collected Diptera (Diptera Neerlandica 1877). Horn \& Kahle r935-'37 p. 308 (Mus. Amsterdam and Mus. Leiden).

$1002 \mathrm{Y}$, see also IJ

1003 YpelaAR, Abraham, Amsterdam. Manufacturer of microscopical objects with Sas. (garden IJzicht on the Hoogendijk). Witkamp I864 II p. 9; Nem nich I809 p. 392 (v 1808).

1004 Zaunslifer, Cabinet of divers naturalia sold Amsterdam i75I (Vosmaer 1790).

1005 Zeeuwsch Genootschap, see Middelburg.

1006 Zijverts, see Zyverts.

1007 Zöllner, A. J., Rotterdam. Coleoptera. Everts 1922 p. XII. R.

ioos Zuijlen van Nijevelt. This probably is the name in the 7 th Catalogue of the Miscellanea Meuscheniana, a Catalogue of the Museum of v.Z.v.N., quoted as Nyvrelt, Nywrelt, Nuvrelt, sold 9. IX. I773 Amsterdam (Cobres I782 I p. I38, I39; Boehmer I785-'89 I).

I009 Zuilen. (Utrecht), Museum "Natura" founded I937 (Musea I938 p. 29I $\left.n^{\circ} 239\right)$.

roio Zulcon, DE, lived in 's Gravenhage and showed Monconys (I666 II p. I45, v I663) a curious birds nest from Zulcon in France.

IOI Zumbag de Koesfelt. His collections were bought by the Leiden University (Siegenbeek 1829 II p. 9I). His cabinet was offered for sale in 1780 , Leiden (Vosmaer r790 especially mentions minerals).

IOI 2 Zürcher F. W., Shells. R. (part to L. Muller).

1013 Zyverts, Walich, druggist, Amsterdam. He seems to have published the life history of the bumble bee in Clutius Byenboeck (Leiden I597, A).

IOI4 Zwolle. Overijsselsch Geschiedkundig Museum contains since I906 the collection of natural history from the "Reventer". (Birds given to Denekamp) (Musea 1938 p. 294). 


\section{LITERATURE}

For significance of letters see p. 3. Though I am sure most books can be easily found in any library, I have given the place where I found them for the sake of convenience. AA, A. J. VAN DER, 1852-'78. Biographisch Woordenboek der Nederlanden. Haarlem. [A in $4^{\circ}$, I have used this illustrated edition; UBA in $8^{\circ}$ ].

AcQUET, H. $\mathrm{D}$, \pm 1708. (A collection of drawings). "Ad exemplaria naturalia summo studio ultra quinquaginta annos ex universis terrarum oris quaesita et in sua collectione conservata pingere curavit". "Post mortem hujus collectionis publica auctio habita est in aedibus defuncti Delfis die Martis 29 Maii 1708 et seq." [M. S. in Library Koloniaal Instituut Amsterdam].

Admiral, J. L', 1774. Nauwkeurige waarnemingen omtrent de verandering van vele Insecten of gekorvene Diertjes, die in omtrent 50 jaaren, zoo in Vrankrijk, als in Engeland en Holland by een verzameld, naar 't leven konstig afgetekend, etc. Amsterdam (edited posthumously by Houttuijn). [A].

AIkIN, J., 1920. A short tour through part of Holland and Flanders, taken by J. Aikin in I784. in: Notes and Queries (12) VII p. I8I, 20I [UBA] (Jensen Suppl. 30).

Andersen, H. C. 1855. Mit Livs Eventyr. Samlede Skrifter XXI-XXII, København. p. 405. [UBA] (Jensen 325).

ANonymus, 1767. Conchyliologie nouvelle et portative ou collection de Coquilles propres à orner les Cabinets des Curieux de cette partie de l'Histoire Naturelle mises par ordre alphabétique avec les notes des endroits d'où elles se tirent et des cabinets qui renferment les plus rares. Paris. [Library J. R. le B. Tomlin, St. Leonards on Sea].

Anonymus, 1790. A Tour in Holland in MDCCLXXXIV by an American. Worcester. [UBA] (Jensen Suppl. 29).

Anonymus, I792. Bemerkungen auf einer Reise nach Holland im Jahre 1790. Oldenburg. [UBL] (Jensen 207).

Anonymus, I8I8. Billets in the Low Countries 1814-'I7. In a Series of Letters. London. [UBA] (Jensen 243).

[KB] (Jensen 26r).

Anonymus, 1819 . Tour in Holland in the year MDCCCXIX. London (without year).

Anonymus, 1897. Wenewitinow M. A. Roesskie w Gollandii. Welikoe Posoljstwo I6971698. Moskwa. In this publication the so called Notebook: Zapisnaja Knizjka ljoebopytnych zametsjanij welikoj osoby... St Petersburg 1788. (Notebook for noting the curious observations of a person of high standing, who 1697-'98 travelled under the name of a nobleman of the Russian Ambassy, also found in Moskowki Westnik 1830 VI and Otetsjestwennyja zapiski 1846-48) p. I99--203: Prilosjenie IV Otrywok iz zapisnoj Knisjki welikoj osoby. (cf Raptschinsky Jaarboek Amstelodamum I936 p. I60 (Jensen Suppl. 12).

(Argenvill.e, Desalliers d'), 1742. L'Histoire naturelle éclaircie dans deux de ses parties principales, la lithologie et la conchyliologie... par M. *** de la Soc. R. d. Sc. de Montpellier. Paris, [Library of Miss Tera van Benthem Jutting].

(—), I757. Idem, Nouvelle edition... par $\mathbf{M}^{* * *}$ des Sociétés $\mathrm{R}$. d. Sc. de Londres et de Montpellier. Paris [A].

- 1780. La Conchyliologie... 3me édition, dédié au Roi par MM de Favanne de Montcervelle, Père et Fils. I. Paris [A].

Arlincourt, Vicomte n', I842. Le pélerin, 3 tom. Paris. [UBA]. (Jensen 304).

BARdT, C. F., I79I. Geschichte seines Lebens, seiner Meinungen und Schicksale. Berlin, Bd. III. [UBA] (Jensen Suppl. 25).

(Barrow, J.?), 183I. A Family Tour through Sth Holland up the Rhine and across the Netherlands to Ostend. London. (The Family Library XXIII for 1832). [KB] (Jensen Suppl. 48).

BeckmanN, I912. Johann... 's Dagboek van zijne reis door Nederland in 1762. Medeged. door G. W. Kernkamp, in: Bijdragen en Mededeelingen Historisch Genootschap Utrecht. 33. p. 3II [UBA] (Jensen I46). 
BeEts, A., 1932-'33. Deensch familiebezoek in Leiden in Mei 1936. Jaarboekje voor geschiedenis en oudheidkunde van Leiden en Rijnland, p. 5 I (translation from: Nyegaard 1929) [UBA].

BestoezJew, N., 1821. Zapiski o Gollandii I815 goda. St Peterboerg. [UBA]. (Jensen Suppl. 40).

Biema, E. van. 1910. Een reis door Holland in 1736 meegedeeld, in: Oud Holland, p. 77. [UBA] (Jensen 125).

BIRKEN, S. voN. 1668. Hoch Fürstlicher Brandenburgische Ulysses... Bayreuth [UBL] (Jensen 55).

BJöRnståHL, J. J., I782. Resa till Frankrike, Italien, Sweitz, Tyskland, Holland, Aengland, Turkiet och Grekeland, beskrifvan af och efter Jac. Jon. Björnståhl. Efter des Död utgifven af Carl Christ. Gjörwell. Del I-VI. Stockholm 1780-'84. I have consulted a German translation: Jacob Jonas Björnståhls Briefe... Leipzig und Rostock, Bd. V, 1782: Nachrichten von seinen ausländischen Reisen [UBA] (Jensen I68). See also: Bijdr. en Meded. Hist. Gen. Utrecht 3I, I910, p. 329 note.

Blainville, DE. 1743. Travels through Holland, Germany, Switserland... Translated from the author's M. S. by G. Turnbull \& W. Guthrie. 3 vol. London. Vol. I. [KB]. (Jensen 102).

Blasius, W., I880. Oeffentliche Anstalten für Naturgeschichte und Alterthumskunde in Holland... Reiseskizze vorgetragen im Verein für Naturw. in Braunschweig in Dez. I879. [A].

Bocage, 1764. Recueil des Oeuvres de Mme du Bocage. Lyon. vol. III [UBU] (Jensen I33).

Boccone, 1764. Kecherches et observations naturelles de M. Boccone, Gentilhomme Sicilien. Amsterdam. [UBA] (Jensen Suppl. 10).

Boddaert, P. I770. Dierkundig Mengelwerk... Utrecht. Translation of Pallas I7662 [A].

- 1768. Lijst der Plantdieren... Utrecht. Translation of Pallas 17661 [A].

Boenmer, G. R., I785. Bibliotheca scriptorum Historiae Naturalis... 5 parts, 9 vols. Lipsiae I785-'89. Vol. I p. 369. Museographi [A].

Bowrey, 1927. The Papers of Thomas Bowrey, r669-1713... edited by R. C. Temple. Works Hakluijt Society (2) $\mathrm{n}^{\circ}$ 58. London [UBA]. (Jensen Suppl. 13).

Brereton, W., 1844. Travels in Holland, the United Provinces, England, Scotland, Ireland, I634-35. Remains Histor. \& Literary, publ. by the Chetham Soc. I [Arch.] (Jensen 38$)$.

British Museum I906. The History of the Collections contained in the Natural History Departments of the... London Vol. II.

Brom, G., 1915. Een italiaansche reisbeschrijving der Nederlanden (1677-'78). Reis van Guido en Giulio de Bovio uit Bologna. Bijdragen en Meded. Hist. Gen. Utrecht. 36, I915, p. 8I [UBA] (Jensen 76).

Brown, E., I082. Naukeurige en gedenkwaardige Reysen van Edward Brown... Vert. door J. Leeuw. Amsterdam [UBA] (Jensen 65).

Calixtus, 1782. Auszug aus der handschriftlichen lateinischen Beschreibung einer Reise des vormaligen Helmstedtschen Gottesgelehrten Friedrich Ulrich Calixtus durch Italien, Frankreich und die Niederlande vom 2Isten April 165I bis zum I7ten Dezember desselben Jahres. Joh. Bernouilli's Sammlung Kurzer Reisebeschreibungen. Bd. VIII, I782 p. 281.

Camper, C., 188I. Catalogue des Manuscripts de Pierre Camper et de Lettres inédites... qui se trouvent dans la Bibliothèque de la Soc. Néerl. pour les progrès de la Médecine à Amsterdam. [This Library now in UBA].

Capelle, J. P. vaN, 182I-'27. Bijdragen tot de geschiedenis der Wetenschappen en Letteren in Nederland. 2 vols. Amsterdam \& Haarlem. [UBA].

Carter, H. W., 1819. A short account of some of the principal hospitals of France, Italy, Switserland and the Netherlands... London. [UBA] (Jensen 258).

Chemnitz, J. H., r760. Kleine Beyträge zur Testaceotheologie... Frankfurt und Leipzig. [A]

Clausade, A., (1835). Feuilles de Voyage, Belgique, Hollande, Ouest de l'Allemagne... Paris [UBA] (Jensen Suppl. 52). 
Cobres, J. P., 1782. Deliciae Cobresianae. Büchersammlung zur Naturgeschichte. 2 parts. 6 vol. Augsburg. $[A]$.

CogAn, T., 1794. The Rhine or a Journey from Utrecht to Francfort. London. Vol. I [KB] (Jensen 209).

ColE, F. J., 1914. History of the Anatomical Museum, in: "A Miscellany presented to J. M. Mackay Ll D." p. 302 [in my possession].

Collot D'Escury, H. Baron, 1844. Holland's Roem in Kunsten en Wetenschappen VII. 's Gravenhage en Amsterdam. [UBA].

Courtanvaux, Marquis DE, 1768 . Journal du voyage sur la frégatte l'Aurore... Paris. [UBU] (Jensen 152).

Coyer, l'abbé, 1775. Voyage d'Italie et de Hollande. II. Paris [UBA]. (Jensen I55).

CRAMER, P., 1779-'82. De uitlandsche Kapellen. Amsterdam I 1779, II 1779, III 1782, IV 1782. [A].

Czermak, J. N., 1879. Gesammelte Schriften. In 2 Bde (9 Thle). Leipzig. I, p. 164-175. [UBA] (Jensen 328).

Desjorert, L., 1910. Voyage aux Pays Bas en 1778, ed. Vic. de Grouchy in: De Navorscher LIX p. 8, 9, 195, 204, 206, 340, 344. [UBA]. (Jensen 176).

Dethmar, F. W., 1838-'4I. Freundliche Erinnerung an Holland und seine Bewohner. 4 Stücke. Essen-Rotterdam. [UBA, Part III also separate as: Dethmar, Reise von Amsterdam in die Nordöstlichen Theile des Königreichs der Niederlande. 1840]. (Jensen 305).

Dryander, J., 1798-1800. Catalogus Bibliothecae Historico-Naturalis Josephi Banks. Londini. 5 vol. [A].

DuchesNe, 1834. Voyage d'un iconophile... Paris [UBA]. (Jensen 276).

Dusseau, J. L., 1865. Catalogue de la Collection d'anatomie... de G. et W. Vrolik. Amsterdam. [A].

Ehrhart, J. F., 1783. Meine Reise nach der Graffschaft Bentheim und von da nach Holland, nebst der Retour nach Herrenhausen. Hannoverische Magazin 1783 (12e-19e Stück) Kolom 177-296 [UBA]. (Jensen Suppl. 28).

EIjNDHOVEN, I861. Catalogue d'une collection très étendue et bien conservée d'Insectes et d'autres objets d'histoire naturelle... délaissé par feu Mr. A. J. van Eyndhoven à Zutphen, $14 \mathrm{X}$ 186I seq. [A, E].

Ekama, C., 1874. Catalogus van boeken, pamphletten etc. over de geschiedenis van Haarlem etc. Ie stuk II88-i800. Haarlem [Archives Haarlem].

EngelmanN, W., 1846. Bibliotheca historico-naturalis 1700-1846. Leipzig [A].

-, 186I. Bibliotheca zoologica (with V. Carus) 2 vol. 1846-'60. Leipzig. [A].

-, 1887-99. Bibliotheca zoologica I86I-'80 (by Dr. O. Taschenberg). [A].

E(RNDTEL), C. H. D(r med.) I7II. De itinere suo anglicano et batavo, annis 1706 et 1707 facto. Amsterdam. [UBA]. [English edition: The Relation of a Journey into England and Holland by a Saxon Physiciar. London 171r]. (Jensen 103).

Evelyn, J. [18\%9]. Memoirs of John Evelyn, comprising his Diary 1641-1705-6... ed. W. Bray. London. [UBA]. (Jensen 4I).

Eversman, F. A. A., I792. Technologische Bemerkungen auf einer Reise durch Holland. Freyberg und Annaberg. [KB]. (Jensen 190).

EverTs, Jhr. E., 1922. De schildvleugelige insecten. 's Gravenhage (I I898, II 1903), III 1922. [A, E].

FelLer, Abbé DE, 1823. Itinéraire ou voyages de M. l'abbé de Feller en diverses parties de l'Europe. 2 me éd. 2 tom. Paris. [UBA]. (Jensen 170).

FERrNER, B., 19I0. Dagboek van zijne reis door Nederland in 1759, medegedeeld door G. W. Kernkamp. Bijdragen en Mededeelingen van het Historisch Genootschap te Utrecht XXXI, 1910, p. 314. [UBA]. (Jensen I43).

Fliedner, Th., 1831. Collektenreise, 2 Bde., [UBA]. (Jensen 268).

FOKKER, A. \& J. C. DE MAN, Igor. Levensberichten van Zeeuwsche medici. Middelburg. [A].

Bijdragen tot de Dierkunde, Af. 27 
Forster, G., 1843. Sämmtliche Schriften, hrsg. v. dessen Tochter... 9 Bde. Leipzig. VII Bd. p. 88 seq. [UBA]. (Jensen Suppl. 26).

—, 1868. Ansichten vom Niederrhein, von Brabant, Flandern, Holland, England und Frankreich im April, Mai und Juni I790. Mit Einleitung und Anmerkungen hrsg. von W. Buchner. Leipzig. II p. 65 seq. [UBA]. The same: Berlin 1793,3 vol, II p. 256 seq. [UBA]. (Jensen 206).

France, J. Le... van Berkhey, I760-I8Ir. Natuurlijke Historie van Holland. Amsterdam \& Leyden. [A].

_-, 1773. Sermo inauguralis de urbe Lugduno Batavorum... Lugd. Bat. [A].

Frisch, née Tutein, P. D., 1816. Reise durch Deutschland, die Schweitz und Italien in den Jahren I797, I 803 und I804. Altona. [Kon. Bibl. Kopenhagen. Kind communication of Dr. Mortensen].

Fuchs, 19I6. Aus dem Itinerarium des Christian Knorr von Rosenroth. Jaarboek Amstelodomum XIV. [UBA, sep. A].

Garampi, r889. Viaggio in Germania, Baviera, Svizzera, Olanda, Francia compiuto negli anni 176I-'63. Diario del Cardinale Giuseppe Garampi (scritti per Gaetano Marini) ed. G. Palmieri Roma. [UBL]. (Jensen 145).

GARAMPI, 1899. Monsignore Garampi in Holland im Jahre 1764. Mitteilung von Fr. vor Weech. Bijdragen en Mededeelingen Hist. Gen. Utrecht. XX. [UBA]. There is also an Italian edition 1889. (Jensen 148).

GedenkвoEK, 19I9. Zeeuwsch Genootschap 1769-I9I9. Middelburg I9I9. [A].

Gedenквоек, 1932. van het Athenaeum en de Universiteit van Amsterdam 1632-I932. Amsterdam [A etc.].

Geijskes, D. C. \& C. J. van Der KllaAuw, 1934. Der heutige Zustand der anatomischen Kabinette früherer Jahrhunderte in Leiden in: Janus XXXVIII.

(Gersaint, E. F.), 1736. Catalogus conchyliorum, Paris. [A].

Geuns, S. J. van, I796. Catalogus librorum... addita... Libraria quam reliquit Petrus Boddaert... Utrecht. [UBA].

Goethe, W. und K. F. Zelter, 1913, Der Briefwechsel zwischen... Im Auftrag des Goethe- und Schiller-Archivs nach den Handschriften. hrsg, v. Max Hecker. 4 Bde, Leipzig. Vol. II [UBA]. (Jensen 269).

Gool, J. vaN, I750-'5I. De nieuwe Schouburg der Nederlandsche kunstschilders en schilderessen. 's Gravenhage. 2 dln. [UBA].

(Grimm, J. F. C.) I775. Bemerkungen eines Reisenden durch Deutschland, Frankreich, England und Holland in Briefen an seine Freunde. III. Altenburg [UBA]. (Jensen I63).

Gronovius, L. Th., I760. Bibliotheca Regni Animalis. Lugd. Bat. [A].

Guislain, J., 1842. Lettre médicale sur la Hollande adressée à M. M. les Membres de la Soc. de Médecine de Gand. Annales de la Soc. de Médecine de Gand [UBA]. (Jensen 317).

GunN, H. ...-Turner, 1834 . Letters written during a 4 days tour in Holland in the summer of 1834. Not published. (Jensen Suppl. 51).

Hagen, H. A. 1862. Bibliotheca Entomologica. 2 Vol. Leipzig [A].

Haller, A. 1883. Tagebücher seiner Reisen nach Deutschland, Holland und England, hrsg. v. L. Hirzel. Leipzig [UBA]. (Jensen II6).

Hanway, J., 1753. An historical Account of the British Trade over the Caspian Sea with a Journal of Travels... Vol. II. London [UBL]. (Jensen 134).

Haupt, Th. von, 1814. Malerische Reisen durch Holland... Neue Ausg. 2 Bde. Hamburg. [UBU]. (Jensen 238).

Hegenitius, G., 1630. Itinerarium Frisio-Hollandicum (et Abr. Ortelii Itinerarium GalloBrabanticum), Lugduni-Bat. [UBA]. (Jensen 35).

Heinecken, K. H. voN, I769. Beschreibung einer Reise nach Niedersachsen, Westphalen und Holland. Nachrichten von Künstler und Kunstsachen II. [KB]. (Jensen I54).

Hil.l, R., I8I6. Sketches in Flanders and Holland. London. [UBA]. (Jensen 247).

Hoffmann von Fallersleben, 1868. Mein Leben. Aufzeichnungen und Erinnerungen. 6 Bde. Hannover. [UBA]. (Jensen 260). 
Holman, J., 1833. The narrative of a journey. 5th ed. London. [UBA]. (Jensen Suppl. 44).

(HonorE, S. F.), I779. La Hollande au I $8 \mathrm{me}$ siècle ou nouvelles lettres contenant des remarques et des observations sur les principales villes, la religion, le gouvernement,... des habitants de cette province. La Haye [UBA]. (Jensen 173).

HoogewerfF, G. J., 1919. De twee reizen van Cosimo de Medici, prins van Toscane door de Nederlanden (1667-'69). Werken Hist. Gen. Utrecht (3) 4I. Amsterdam. [UBA]. (Jensen Suppl. 8).

HorN, W., 1831. Reise durch Deutschland, Ungarn, Holland... Bd. I. Berlin. [UBA]. (Jensen 279).

HoRN, W. \& I. Kahle, 193.5-'37. Uber Entomologische Sammlungen, Entomologen und Entomo-Museologie. I-III. Entomologische Beihefte Bd. 2--4, 1935, 1936, I937 [E].

Horst, R., \& M. M. Schepman, 1908. Muséum d'Histoire Naturelle des Pays Bas. Catalogue systématique des Mollusques. 3me Partie (herewith: Introduction). Leiden [A].

Houtruyn, M., I76I-'73. Natuurlijke Historie of uitvoerige beschrijving der dieren, planten en mineraalen volgens het samenstel van den Heer Linnaeus. Amsterdam. Deel I, 18 stukken [A].

Huber, Th. H., I8II. Bemerkungen über Holland, aus dem Reisejournal einer deutschen Frau. Leipzig. [UBA]. (Jensen 237).

Hublard, E., 1910. Le naturaliste hollandais Pierre Lyonet. Sa vie et ses oeuvres (I7061789)... Bruxelles. [A].

HUDE, H. VAN DER, 1784 . Reise durch Holland 1755 , in : Bernouilli's Sammlung kurzer Reisebeschreibungen... XIII \& XIV. [Kön. Bibl. Berlin, kindly communicated by Prof. W. Arndt]. (Jensen I37).

IrELAND, S., I790. A picturesque tour through Holland, Brabant and part of France, made in the autumn of 1789. I. [Arch.]. (Jensen 205).

Jacobaeus, H. I9I0. Rejsebog (I67I-'92). København [Library Nederl. Tijdschr. v. Geneesk.]. cf. Mendels, Ned. Ts. v. Geneesk. 82, I938, p. 153.

JAECK, J. H. I826. Reise durch Frankreich, England und die beiden Niederlande im Sommer und Herbst I814. 2 Thle in I Bd. Weimar [UBL]. (Jensen 270).

Jensen, J. N. Jacobsen, 1919. Reizigers te Amsterdam... Amsterdam, Supplement 1936. [UBA].

Joly, 1672. Les voyages de Munster, d'Hollande, d'Osnabrugh, Varendorph, des PaisBas, et de Cologne. Paris. [Arch.]. (Jensen 44).

JokdAN, F. I., 19I8. Zapiski rektora i professora akademii goedosjest Fedora Iwanowitsja Jordan [S. predislowiem S. P. Winogradowa]. Moskwa. [UBA]. (Jensen Suppl. 50).

Jordan, C. E., J735. Histoire d'un voyage littéraire fait en 1733 en France, en Angleterre et en Hollande. La Haye. (Jensen I23).

KalfF, S., 1928. Indische Conchylien. De Natuur. [A].

KALFF, M., 1875. Amsterdam in plaatjes en praatjes, 2 dln I Bd. [UBA].

KANold, J. \& C. F. Neickelius (= Einckel) 1727. Museographia. oder Anleitung zum rechten Begriff und nützlicher Anlegung der Museorum oder Raritäten Kammern. Leipzig und Breslau. [KB].

KerBert, C. I888. Het Aquarium en zijne bewoners beschreven en toegelicht. Bijdragen tot de Dierkunde. Feestnr. 50 j. bestaan. Amsterdam. [A].

Kerbert, C., I898. Het Koninklijk Z.oölogisch Genootschap “Natura Artis Magistra” I838I Mei 1898. [A].

Kerbert, C., 1905. Het Aquarium te Amsterdam. Amsterdam, voor Mij tot Nut van het Algemeen (bij van Looy). [A].

Kerbert, C., 1906. L'Aquarium de la Soc. R. de Zool. "Natura Artis Magistra" à Amsterdam. Amsterdam. [A].

Kerbert, C. 1926. Toespraak gehouden bij de opening van het Zoölogisch Museum en het "Heimans Diorama" in het Aquariumgebouw op Donderdag 20 Mei 1926. Amsterdam. Uitgave K. Z. Gen. "N.A.M.". [A].

KLAAUW, C. J. VAN DER, 19261. Het hooger onderwijs in de zoologie en zijn hulpmiddelen te Leiden. Leiden. 
KLAAUW, C. J. VAN DER, I9262. De biologische afdeeling van het Nederlandsch Historisch Natuurwetenschappelijk Museum te Leiden. in: Vakblad voor biologen, XII, p. I89. [A].

KLAAUW, C. J. VAN DER, 1930. Een verzameling uit den eersten tijd van de vergelijkende ontleedkunde: in: "De Natuur". p. 49-53, 73-78, 97-ror, 122-126. [A].

KluijtenaAR, H. A., 1936. Amsterdam en zijn bewoners in de 70, 80er jaren van de vorige eeuw. Jaarboek Amstelodamum 33, 1936, p. 249 [UBA].

KNORR, G. W., I770-'75. Verlustiging der oogen en van den geest of verzameling van allerley bekende Hoorens en Schulpen. Amsterdam, I-VI. [A].

Koerakin, 3890. Archiwj Kn. F. A. Koerakina Izdaw. pod. red. M. I. Semewskawo Kniga perwaja. Boemagi Knjazja Borisa Iwanowitja Koeiakina 1676-1727. St. Peterboerg. [UBL] [see: Raptschinsky in: Jaarboek Amstelodamum 33, 1936, p. I65]. (Jensen Suppl. 15).

(LAdenberg, P. W. A. voN), I830. Kurze Bemerkungen auf einer flüchtigen Reise am Rhein und durch das Königreich der Niederlande im Jahre 1828. [UBA]. (Jensen 277).

LAmy, E., 19.30. Les Cabinets d'Histoire naturelle en France au XVIIIe siècle et le Cabinet du Roi (I635-1793). Paris chez l'auteur. [Library Rijksmuseum Nat. Hist. Leiden, Moll. 1082].

LAx, L., 1838 . Bilder aus den Niederlanden II. Aachen. [UBA]. (Jensen 302).

LeмpкE, B. J., 1936. Catalogus der Nederlandsche Macrolepidoptera. Tijdschr. voor Entomologie 79, p. 238. [E].

Lennep, J. van, 1862. Het leven van Mr Cornelis en Mr David Jacob van Lennep. Amsterdam [UBA].

Lennep, M. F. van, I9Io. Het leven van Mr Jacob van Lennep. 2e druk. Amsterdam 2 dln. [UBA].

Lesser, F. C., 1744. Testaceo-Theologia. Leipzig. [A].

LESSER, F. C., I756. Idem. 2e Ed. Leipzig. [A].

Lidth DE Jetrne, Th. G. van, 18.58. Catalogue du Musée Zoologique de ... Arnhem [A, B].

Liefrinck, M., 1925. Odonata Neerlandica.Tijdschrift voor Entomologie. LXVIII, p. 6r. $[\mathbf{A}, \mathbf{E}]$.

LoISEL, G. 1907. Rapport sur une mission scientifique dans les jardins et établissements zoologiques publics et privés du Royaume-Uni de la Belgique et des Pays Bas in: Nouv. Arch. des Missions scientif. XIV Paris. [A].

LoIsei., G., I9I2, Histoire des ménageries de l'antiquité à nos jours. Paris. 3 Vol. [A].

LUC, J. A. DE, I779. Lettres physiques et morales sur l'histoire de la terre et de l'homme. Paris et la Haye. t. III. [Libr. Teyler, Haarlem]. (Jensen 172).

LucaE, F., 1854. Der Chronist Friedrich Lucae... Frankfurt am Main [UBL] (Jensen 6I).

LuGT, F., 1938. Répertoire des Catalogues de Ventes publiques intéressant l'art ou la curiosité... Ière période: vers I000-1825. La Haye. [B and Rijksmuseum Amsterdam].

L(yNAR), R. F. Graf zu, I78I. Reise durch Holland im Jahr 177I. in: Joh. Bernouilli's Samml. kurzer Reisebeschr... I p. 95 [Kön. Bibl. Berlin, kindly communicated by Prof. W. Arndt]. (Jensen 157).

MAC Gregor, J., 1835. My note book, London. Vol. I. [Archives Leiden]. (Jensen 290).

Maitland, R. T., I855. De verdiensten der Nederlanders ten opzichte van de Beoefening der Dierkunde. Jaarboekje van het Kon. Zool. Gen. "Natura Artis Magistra". p. 159. [A].

Maitland, R. T., 1863. De Musea van het Kon. Zoöl. Gen. "Natura Artis Magistra" op I Mei I863. in: Nederl. Tijdschr, v. d. Dierk. I p. XLIX. [A].

Maitland, R. T., I888. Ontstaan, ontwikkeling en bloei van het Kon. Zool. Gen. "Natura Artis Magistra" te Amsterdam. in: Bijdragen tot de Dierkunde Feestnr. 50-j. bestaan. Amsterdam [A].

Meijer, D. C., I889. Blauw Jan. Amsterdamsch Jaarboekje. p. 4 I [UBA].

Meiszner, E., 1819. Bemerkungen aus dem Taschenbuche eines Arztes während einer Reise von Odessa durch ein Theil von Deutschland, Holland... Halle. [Archives Rotterdam]. (Jensen 252). 
Melle, J. von \& C. H. Postes., I89I. Beschreibung einer Reise durch das nordwestliche Deutschland nach den Niederlanden und England im Jahre 1683. Hrsg. v. C. Curtius. Lübeck. Schulprogr. [Arch.]. (Jensen 87).

Merck, J. H., I9Ir. Briefe an die Herzogin-Mutter Anna Amalia und an den Herzog Carl August von Sachsen Weimar. hrsg. v. H. G. Graef. Leipzig. [KB]. (Jensen I92).

Meuschen, F. C., I766-'7o. Miscellanea Conchyliologica... (a collection of different sale catalogues all prepared by Meuschen). [A not complete].

Misson, 1722. Voyage d'Italie de M. Misson. 5e éd. Utrecht. t. I. [UBA]. (Jensen 90).

Moes, E. W., I899. Vorstelijke bezoeken te Amsterdam in: Amsterdamsch Jaarboekje. p. I [UBA] [about: Itinerarium der Reise von Kassel aus in Englandt. A. I6I I den I4 Maji angefangen, und den 4 Sept bris glücklich volendet, M. S. in Landesbibl. Kassel].

Molhuysen, P. C., P. J. Blok \& F. K. H. Kosmann, Ig.II-'37. Nieuw Nederlandsch Biografisch Woordenboek. Leiden. I-X. [UBA].

Monconys, 1665 \& I666. Journal des Voyages de M. de Monconys... publ. p. le Sieur de Liergues son fils, I. II. Lyon [UBA]. (Jensen 60 ).

Monsanto, A., 1752. A tour from England thro' part of France, Flanders and Holland. London. [British Museum, kind communication of $\mathrm{Mr} \mathrm{R}$. G. Rendall]. (Jensen I35).

Moryson, F., 1617. An itinerary. London. [UBA] (see Jensen in: Jaarboek Amstelodamum XIII ; Koster in: Groot Nederland, Aug. I914]. (Jensen 15).

Motraye, A. De LA, 1727. Voyages du Sr. A. de la Motraye en Europe, Asie et Afrique. II. La Haye [UBL]. (Jensen 107).

Mountague, W., 1606. The delights of Holland or a three months Travel about that and the other provinces. London. [UBA]. (Jensen 94).

Mundy, P., 1925. The Travels of Peter Mundy in Europe and Asia 1608-'17, ed. by R. C. Temple. Vol. IV. London. Hakluijt Soc. (2) L.V issued for 1924. [UBA]. (Jensen Suppl. 4).

Muntendam, A. M., 1938. Martinus van Marum, Docteur ès sciences. in: Archives du Musée Teyler (3) VIII p. 464

Murrax, D., 1904. Museums, their History and their Use... Glasgow. 3 Vol. [UBA].

MusEA, 1938, de Nederlandsche, Uitgegeven vanwege het Departement van Onderwijs, Kunsten en Wetenschappen. 's Gravenhage. [Library Zoöl. Mus. Amsterdam].

Nagtglas, F., 1888-'93. Levensberichten van Zeeuwen..., 4 afl. Middelburg. [A].

NEILL, P., J823. Journal of a Horticultural Tour through some parts of Flanders, Holland and the North of France in the autumn of 1817. Edinburgh [Library Landbouwhoogeschool, Wageningen]. (Jensen Suppl. 41).

Nemnich, Ph. A., I809. Tagebuch einer der Kultur und Industrie gewidmeten Reise. III and IV. Tübingen. [UBA]. (Jensen 235).

Neumayr, J. W. von Ramszla, I620. Des durchlauchtigen... Fürsten... Johann Ernsten... zu Sachsen... Reise in... Niederland. Leipzig [UBA] (Jensen 26).

Niebuhr, B. G., 1842, Nachgelassene Schriften nichtphilologischen Inhalts. Hamburg [UBA]. (Jensen 234).

Niemeyer, A. H., 1823. Beobachtungen auf Reisen in und ausser Deutschland... Bd. III, Reise durch... Holland im Jahre I806. Halle [UBA]. (Jensen 233).

Nierop, L. van, 1932. Naamregister van alle de voornaamste winkeliers der stad Amstelredam, met aanwijzing derzelver woonplaatsen... voor het jaar I767. Jaarboek Amstelodamum XXIX, p. 159. [UBA].

Nozeman, C., I770, Nederlandsche Vogelen... Alle naer 't leven... getekend... door en onder toezicht van Christiaan Sepp en Zoon. Amsterdam. [A].

Nuijens, B. W. Th., I928. Het ontleedkundig onderwijs en de geschilderde anatomische lessen van het Chirurgijnsgilde te Amsterdam in: Jaarverslag in de 7oe Alg. Verg. Oudh. Genootschap, 2I Mei 1928, p. 45.

NyegaARD, P. N., 1920. En selvbiografi. Odensee, [UBA] (cf. Beets).

OGIER, Ch., rgro. Reiseeindrücke aus Danzig, Lübeck, Hamburg und Holland, 1636. Nach dem neuentdeckten II. Teil von Charles Ogiers Gesandschaftstagebuch. Bearb. und hrsg. v. Kurt Schottmueller. Zeitschr. d. westpreus. Geschichtsvereins. Heft 52, p. 199 [Bibliotheca Rosenthaliana in UBA]. (Jensen 39). 
Oliver, W., 1703. A letter giving his remarks on a late journey into Denemark and Holland. Philos. Transact. R. Soc. London $n^{\circ} 285$ in Vol. 23, p. 1400 [Kon. Akademie]. Oujvier, W. J., I838. Manuel des étrangers à Amsterdam [UBA].

Oтто, C., 1825. Reise durch die Schweiz... Holland... Hamburg, II. [UBA]. (Jensen 266).

Pacichelli, G. B., 1684. Memorie de viaggi per l'Europa christiana, scritti a Diversi in occasione de suoi ministeri. Bd. I. [Kön. Bibl. Berlin, kind communication of Prof. W. Arndt]. (Jensen 72).

Pallas, P. S., r7661. Elenchus zoophytorum... Hagae Comitis. [A].

-, I7662. Miscellanea zoologica... Hagae Comitis [A].

-, 1767-'77. Specilegia zoologica... Berolini [A].

P(ATiN), C., I676. Relations Historiques et Curieuses de Voyages, en Allemagne, Angleterre, Hollande... Par C. P. D. M. de la Faculté de Paris. Rouen. [UBA]. (Jensen 70).

Payen, 1663. Les voyages de M. Payen. Paris. [UBA]. (Jensen 53).

Pekarskı,, P., 1862. Naoeka i literatoera w Rossii pri Petrje Welikomj. 2 Vol. S. Peterboerg. [UBL]. (Jensen Suppl. 17).

Percy, E., 1775. A short Tour, made in the year thousand seven hundred and seventy one London. [Brit. Mus. Kind communication of Mr Rendall]. (Jensen Suppl. 22).

Phelsum, M. van, 1774. Brief aan den... Heere Cornelius Nozeman... over de Gewelvslekken... Rotterdam. [A].

(PICTET, J.), I827. Notes sur la Nord Hollande (Juin I822) par un voyageur suisse. Bruxelles. [UBA] .(Jensen 265).

Poellnitz, C. L. DE, I734. Mémoires de Ch. L. de Poellnitz contenant les observations qu'il a faites dans ses voyages... Liège III [UBA]. (Jensen 12I).

Poole, R., 1743. A Journey from London to France and Holland or the Traveller's useful Vademecum. London. Vol. II. [UBA]. (Jensen 127).

RAdCLIFFe, A., 1795. A journey made in the summer of 1794, through Holland... 2nd ed., London Vol. I [KB] (Jensen 2I4).

Raptschinsky, B., 1936. Peter de Groote in Holland in 1697-'98. Een historische schets. [UBA].

Ray, J., 1738, Travels through the Low-Countries, Germany, Italy and France, with curious observations... 2d ed. Vol. I. London. [KB]. (Jensen 57).

Regenfusz, F. M., 1758. Choix de Coquillages et de Crustacés. Copenhague. [A]

Regnard, I874. Voyage de Regnard en Flandre, en Hollande, en Danemark et en Suède. (168I). Nouv. éd. p. A. de Marsy. Paris. [UBA]. (Jensen 84).

Rudolphi, K. A., I804. Bemerkungen aus dem Gebiet der Naturgeschichte, Medicin und Thierarzneykunde auf einer Reise durch einen Theil von Deutschland, Holland und Frankreich I. Berlin. [Kön. Bibl. Berlin kindly communicated by Prof. W. Arndt]. (Jensen 227).

RuE, P. DE LA, 1734. Geletterd Zeeland, verdeeld in drie afdeelingen. Middelburg. [UBA].

RUMPELT, G. L., 1802. Veterinarische und ökonomische Mittheilungen von einer Reise durch... Holland... aus dem Nachlasse... hrsg... v. Riem. Dresden [non vidi, cf. Jensen Suppl. $\mathrm{n}^{\circ}$ 27].

Rumphiús, G. E., 1705. Amboinsche Rariteitkamer... Amsterdam. [A].

SANDER, H., 1783. Beschreibung seiner Reisen... Leipzig. Vol. I. [KB] (Jensen I7I).

SARTre, P., I89I. Voyage en Hollande fait en I7I9... Paris [UBA]. (Jensen II3).

Schaeffer, J. C. G., 1794. Briefe auf einer Reise durch... Holland... in den Jahren 1787 un 1788 geschrieben. II. Regensburg. [UBA]. (Jensen 203).

Schopenhauer, A., 1923. Reisetagebücher aus den Jahren 1803-'04, hrsg. von Ch. von Gwinner... Leipzig [UBA]. (Jensen Suppl. 35).

SebA, A., 1734-'65. Locupletissimi rerum naturalium Thesauri accurata descriptio. Amsterdam. 4 Vol. [A].

SEPP, J. C., I762-I860. Beschouwing der wonderen Gods in de minstgeachte schepzelen of Nederlandsche insecten. Vol I-VIII [A].

(SHAw, J.), I709. Letters to a nobleman from a gentleman travelling thro' Holland... London. [Arch.]. (Jensen 100). 
Siegrnbeek, M., I829. Geschiedenis der Leidsche Hoogeschool 1575-1825. Leiden. [UBA]. (SierstorpFF, C. H. voN), 1804. Bemerkungen auf einer Reise durch die Niederlande nach Paris im IIten Jahre der grossen Republiek. II. Hamburg [UBU]. (Jensen 226).

(Silliman, B.), 1812. A Journal of travels in England, Holland and Scotland... in the years 1805 and 5806 . 2nd ed. Boston. II [UBA]. (Jensen Suppl. 36).

Silva Rosa, J. S. DA, 1927. De bezoeken van Gacham Azulay aan Nederland in de tweede helft der I8e eeuw in: De Vrijdagavond, I en 8 April 1927. [Broch. in Bibl. Rosenthal. in UBA].

SinCERUS, J. (J. Zintzerling), 1649. Itinerarium Galliae.... Amstelodami [UBA]. (Jensen 22).

Smith, J. E., I796. Reise durch Holland, Frankreich und Italien [a. d. Engl. v. G. C. Reich]. I. Leipzig. [Arch.]. (Jensen 198).

Stoll, C., 17881. Natuurlijke en naar 't leeven naauwkeurig gekleurde afbeeldingen en beschrijvingen der Wantzen... Amsterdam (Sepp). [A].

-,$I 788$. Natuurlijke en naar 't leeven naauwkeurig gekleurde afbeeldingen en beschrijvingen der Cicaden... Amsterdam (Sepp). [A].

- I I 13. Natuurlijke en naar 't leven nauwkeurig gekleurde afbeeldingen en beschrijvingen der Spooken, wandelende bladen, zabelsprinkhanen, krekels, trek-sprinkhanen en kakkerlakken. Amsterdam (Sepp). [A].

Stresemann, E., 1923. Die Anfänge ornithologischer Sammlungen. Journal f. Ornithol. LXXI, p. II2.

(Strick van Linschoten, P. H. A. J.), (Eleuterophilos), 1818. Vertraute Briefe während eines Durchflugs durch einen Theil der nördlichen Provinzen des Königreichs der Niederlande im Sommer des Jahres 1817... I. Germania (Mannheim). [UBA]. (Jensen 254).

StruiJck, 17I8. Verschijden uytlandsche Insecten geteekent na het cabinet van d'Hn. Seba, J. ten Kate etc. 6 Vol. gr. fol. [M S drawings, 271 sheets in 6 portefolios, mentioned by Hagen, 1863 II p. 394 (ad p. 203) also in T. O. Weigels Catalog. XIII I862 p. 48, could not be traced furthe ${ }^{3}$.

Suringar, G. C. B., 1866. De Leidsche Geneeskundige Faculteit in het begin der I8e eeuw. Nederl. Tijdschr. v. Geneesk. (2) II. 2 p. I-29. [UBA].

--, 18671. De school van Bernhard Siegfried Albinus. Ned. Tijdschr. v. Geneesk. (2) III 2, p. I [UBA].

- 18672 . Het bijeenbrengen van een verzameling van natuurlijke voorwerpen voor het academisch onderwijs. Ned. Tijdschr. v. Geneesk. (2) III, 2. p. 265. [UBA].

Temminck, C. J., I808-'og. Histoire naturelle générale des Pigeons. Paris. [A. only 9 parts].

Temminck, C. J. \& le B.on Meiffren Laugier de Chartrouse, 1838. Nouveau recueil de Planches coloriées d'Oiseaux. Paris. 5 Vol. [A].

TERWEN, J. L., I855. Het Koninkrijk der Nederlanden... Darmstadt. 2 dln. [A].

Tessin d. y. s., N., 1914. Studieresor i Danmark, Tyskland, Holland, Frankrike och Italien. Anteckningar, bref och ritningar utg. af Osv. Sirén. Stockholm. [KB]. (Jensen 89).

Thourn, A., I84I. Voyage dans la Belgique, la Hollande et l'Italie. I. Paris [Arch.]. (Jensen 216).

Thunberg, K. P. 1792-'94. Reise durch einen Theil von Europa, Afrika und Asien... I770-'79. a. d. Schwed... 2 Bde. Berlin. [UBA]. (Jensen I56).

Tisch Bein, J. H. W., I86I. Aus meinem Leben I. Braunschweig. [KB]. (Jensen Suppl. 23).

Titius, C. H., 1783. Auszug aus dem Reise-Journal von seiner vorzüglich zu Besichtigung fremder Naturalien-Sammlungen in den Jahren 1777 durch Deutschland nach Holland, Engeland und Frankreich angestellten Reise. Ier Abschnitt, Reise durch Deutschland und Holland, in: Joh. Bernouilli's Sammlung Kurzer Reisebeschr... IX, p. 147 (kindly communicated from Kön. Bibl. Berlin by Prof. W. Arndt). (Jensen 174).

UfFenbach, Z. C. von, I754. Merkwürdige Reisen durch Niedersachen, Holland und Engelland. II, III. Ulm [UBA]. (Jensen I06).

Valentijn, F., 1726. Oud en Nieuw Oost-Indiën... Dordrecht-Amsterdam. III. [A].

- 1754. Verhandeling der Zee-horenkens en Zee-gewassen in en omtrent Amboina... Amsterdam. [A]. 
Valentinus, D., I7I4. Musei Museorum oder der Allgemeinen Kunst und Naturalienkammer II tomus... Frankfurt a.M. [UBU].

Velten, J., about 1700. Wonder der Natuer... (M. S. with curious pictures and plates of natural curiosities seen in Amsterdam about 1700, especially in the tavern "Blauw Jan"). [A].

VERYARD, E., I7OI. An account of divers choice remarks... taken in a journey through the Low Countries... London. [KB].

VIJver, C. VAN DER, I848. Geschiedkundige Beschrijving der stad Amsterdam. 4 dln. Amsterdam. [UBA].

Voet, J. E., I806. Catalogus systematicus coleopterorum (following each other Latin, French and Dutch text) and ed. (Ist ed. was 1766). La Haye [A].

Vos, P. D. DE, I93I. De vroedschap van Zierikzee van de tweede helft der I6e eeuw tot I795. Middelburg. [UBA].

VOSMAER, A., I790. Generale catalogus of naamlijsten van zeer vele, zoo in- als uitlandsche verkopingen, namelijk van: I Bibliotheken..., 2 Kabinetten der Natuurlijke Historie, etc. [M. S. in B] (mentioned in Van der Aa 1852-'78, sub voce Vosmaer).

- I766-I804. Natuurkundige beschrijving eener uitmuntende verzameling... leevend geweest op het Kleine Loo. Amsterdam. (Title of 1804: Regnum animale). , I800. Catalogue de livres... délaissé par feu... La Haye [A].

Willebrandt, J. P., 176r. Historische Berichte und praktische Bemerkungen auf Reisen... hrsg. v. G. Schïtze. 3e Aufl. Frankf. und Leipzig [KB]. (Jensen I4I).

Wimmer, C. W., 1826. Beschreibung einer Reise durch das Königreich der Niederlande... von S. von Grouner... I. [UBA]. (Jensen 264).

Witkamp, P. H., 1864. Amsterdam in schetsen. II p. 107-I50, [UBA, Sep' in A, N.B. I have generally given the pages of this separate, in some cases they could be changed in the original pagination].

$\longrightarrow, 1872$, in: H. Schlegel, De dierentuin van het Kon. Zoöl. Gen. Natura Artis Magistra te Amsterdam. Amsterdam. [A].

- 1875. Het Natura Artis Magistra onzer voorouders in: Jaarboekje van het Kon. Zoöl. Gen. "Natura Artis Magistra". Amsterdam p. 15I [A].

- 1888 . Vroegere diergaarden en beoefenaars der dierkunde. in: Eigen Haard, I888, p. 268-27I. [UBA].

Wright, E., I730. Some observations made in travelling through France, Italy etc. in the years 1720, 1721 and 1722. [UBA] (Jensen Suppl. I8).

Wulp, F. M. van DER, 1895. De Nederlandsche Entomologische Vereeniging. Geschiedkundig overzicht I845-'05. 's Gravenhage [A, E]. 


\section{ADDENDA}

5 bis Ailly, J. DE, see A. J. d'Ailly.

Ad Anonymi Leiden 26. IV. I790, B $\mathrm{n}^{\circ} 556 \mathrm{~b} ; 26$. III. I789, B $\mathrm{n}^{\circ}$ 534 a ; Amsterdam 21. IV. I840, B n ${ }^{\circ}$ I I4 a ; Rotterdam, 24. II. I868, B n० 2122 b; Amsterdam, 3. V. I877, B n 2449 b.

5I bis Berckel, Mr. Gerard. "Commis van de Finantien van Holland”. Cabinet of shells etc., inherited by following.

5I ter Berckel, Mr. Willem. "Raad en Burgemeester der Stad Delft". Cabinet inherited from preceding. Sold Amsterdam 24-III-I76I [Cat. in Prentenkabinet Amsterdam].

5I quater Bergeon, Mr. JaQues. Banker. Spuistraat, 's Gravenhage. Catalogue of sale 28. XII. I789 (B $\left.\mathrm{n}^{\circ} 55 \mathrm{Ia}\right)$.

88 bis Bogaart, Paulus van den, "Schepen en Raad der Stad Delft". Cabinet of shells, sold 22. XII. I8OI (Arch.).

I70 bis Cosijn, A. "Inspecteur bij de marine". Collection to Mus. Leiden (G p. 163 ).

I9I bis Deinse, A. B. van, born Nijmegen 9. XI. I885. Teacher Nat. Hist. Erasmiaansch Gymnasium Rotterdam. Collection on whales and whaling (see his dissertation: De fossiele en recente Cetacea van Nederland, I93 I). R. cf. Rotterdam.

220 bis Drieling. 's Gravenhage. "Referendaris". Collection of shells, etc., partly to Leiden Museum (G p. I64).

309 bis Gravezande, Willem Jacob 's, 27. IX. I688 's Hertogenbosch - 28. II. 1742 Leiden. Prof. Math. astr. Leiden I716, philos. I734. Seems to have had a collection of shells. Valentijn I726 III p. 561 ; I754 p. 45 ; Rumphius I705 p. I33; Valentinus I7I4 II p. I9; Sander I783 I p. 529; Haller I883 p. 37, 107; Kanold I727 p. I95; Van der Aa 1852-'78 IV (b) ; Molhuijsen (b).

3 I9 bis Groote. Collection bought I827 by 'Temminck (G p. I66).

362 bis Hekmeijer. Military apothecary. Collection of insects to Leiden $\mathrm{Mu}$ seum 1872 (G p. I67).

439 bis Kessel, P. van. Med. Dr. Amsterdam. Catalogue of sale (especially anatomy), II. XII. I787 (B $\mathrm{n}^{\circ} 501 \mathrm{I}$ ).

ad 458 Kommer. Catalogue in Mauritshuis or Rijksbureau v. Documentatie.

7I7 bis Roos, P., "Oud-fiscaal van het Committee der Marine". Catalogue of sale, 23-24. IV. I800, Ie.

738 bis Sandra, J., Conservator 's Lands Kabinet van Nat. Hist. Amsterdam. G. p. 5 . 
76r bis Schopman, Mrs. A. G. widow of JB. Spruit, collection of 200 living gold and silverfishes sold the Hague 5. IV. 1787 (B $\mathrm{n}^{\circ} 496$ a).

843 bis Susanna, Johannes Andreas. Leiden II. XII. I795-I. K. I859. Administrator of Leiden Museum. Collection of birds ( $\mathrm{G}$ p. 260 ).

854 bis Tengbergen. Collection of insects to Leiden Museum I87i. G p. J70. 